Draft Version February 28, 2019

Preprint typeset using $\mathrm{L}_{\mathrm{A}} \mathrm{EX}$ style emulateapj v. 12/16/11

\title{
MAGNETOHYDRODYNAMIC-PARTICLE-IN-CELL SIMULATIONS OF THE COSMIC-RAY STREAMING INSTABILITY: LINEAR GROWTH AND QUASI-LINEAR EVOLUTION
}

\author{
Xue-Ning Bai ${ }^{1,2}$, Eve C. Ostriker ${ }^{3}$, Illya Plotnikov ${ }^{3,4}$, James M. Stone ${ }^{3}$ \\ ${ }^{1}$ Institute for Advanced Study, Tsinghua University, Beijing 100084, China \\ ${ }^{2}$ Tsinghua Center for Astrophysics, Tsinghua University, Beijing 100084, China \\ ${ }^{3}$ Department of Astrophysical Sciences, Peyton Hall, Princeton University, Princeton, NJ 08544 and \\ ${ }^{4}$ Université de Toulouse, UPS-OMP, IRAP, 9 av. Colonel Roche, BP 44346, F-31028 Toulouse Cedex 4, France \\ Draft version February 28, 2019
}

\begin{abstract}
The gyro-resonant cosmic-ray (CR) streaming instability is believed to play a crucial role in CR transport, leading to growth of Alfvén waves at small scales that scatter CRs, and impacts the interaction of CRs with the ISM on large scales. However, extreme scale separation $(\lambda \ll \mathrm{pc})$, low cosmic ray number density $\left(n_{\mathrm{CR}} / n_{\mathrm{ISM}} \sim 10^{-9}\right)$, and weak CR anisotropy $\left(\sim v_{A} / c\right)$ pose strong challenges for proper numerical studies of this instability on a microphysical level. Employing the recently developed magnetohydrodynamic-particle-in-cell (MHD-PIC) method, which has unique advantages to alleviate these issues, we conduct one-dimensional simulations that quantitatively demonstrate the growth and saturation of the instability in the parameter regime consistent with realistic CR streaming in the large-scale ISM. Our implementation of the $\delta f$ method dramatically reduces Poisson noise and enables us to accurately capture wave growth over a broad spectrum, equally shared between left and right handed Alfvén modes. We are also able to accurately follow the quasi-linear diffusion of CRs subsequent to wave growth, which is achieved by employing phase randomization across periodic boundaries. Full isotropization of the CRs in the wave frame requires pitch angles of most CRs to efficiently cross $90^{\circ}$, and can be captured in simulations with relatively high wave amplitude and/or high spatial resolution. We attribute this crossing to non-linear wave-particle interaction (rather than mirror reflection) by investigating individual CR trajectories. We anticipate our methodology will open up opportunities for future investigations that incorporate additional physics.

Subject headings: plasmas - magnetohydrodynamics — cosmic rays — instabilities — methods: numerical
\end{abstract}

\section{INTRODUCTION}

Cosmic-rays (CRs) are an essential constituent of the Galaxy. With energy density comparable to or exceeding other components of the interstellar medium (ISM), CRs likely play an important role ISM heating, chemistry, and dynamics over timescales of $\sim 10^{3}-10^{8} \mathrm{yr}$, as well as galaxy formation and evolution over Gyr timescales (see e.g. the reviews of Ferrière 2001; Zweibel 2017; Naab \& Ostriker 2017). The dominant CR population is protons, with $\sim \mathrm{GeV}$ particles representing the peak of the $\mathrm{CR}$ energy distribution; the typical cosmic ray energy density of $\sim 1 \mathrm{eV} \mathrm{cm}{ }^{-3}$ corresponds to a number density $n_{\mathrm{CR}} \sim$ $10^{-9} \mathrm{~cm}^{-3}$ (e.g. Grenier et al. 2015).

CRs are expected to be primarily produced in collisionless shocks, most likely from young supernova remnants (e.g., Baade \& Zwicky 1934), via diffusive shock acceleration (e.g., Krymskii 1977; Bell 1978; Blandford \& Ostriker 1978; Drury 1983). They are then scattered across the Galaxy, primarily following magnetic field lines in the turbulent ISM, and eventually escaping after a few million years (Ginzburg \& Syrovatskii 1964).

Being a non-thermal high-energy particle population, the CRs only infrequently collide with the ISM particles (this is greatest at low energies, causing ionization and heating), but the CRs and bulk ISM gas indirectly inter-

xbai@tsinghua.edu.cn act as they both couple to the interstellar magnetic field. Through this indirect coupling, CRs can exert pressure forces on the ISM gas which are dynamically important. Moreover, being a (trans-)relativistic plasma component, CRs in the ISM are highly buoyant, with a natural tendency to escape from the Galaxy. As a result, the CR component can possibly contribute to large-scale instabilities and dynamo activity (Parker 1966, 1992), and to driving galactic winds (Ipavich 1975; Breitschwerdt et al. 1991; Zirakashvili et al. 1996; Everett et al. 2008, see also below).

At microscopic level, the physics of the interaction between CRs and background thermal ISM gas is extremely rich. Considered as passive test particles, the CRs can be scattered diffusively by background ISM turbulence at the gyro-radius scale (e.g., Jokipii 1966; Schlickeiser \& Miller 1998; Yan \& Lazarian 2002), which is important for understanding CR transport. For typical $\sim \mathrm{GeV}$ CR particles, the gyro-radius for an ISM magnetic field of a few $\mu G$ is on the order of $10^{12} \mathrm{~cm}$ - i.e. a micro-pc. Considering the huge dynamic range from the energycontaining scales of ISM turbulence to the relevant wavelength (a factor of $10^{8}$ in length), as well as the anisotropy of the MHD cascade at sub-pc scales (Goldreich \& Sridhar 1995), it is considered unlikely for background turbulence to be the dominant source of $\mathrm{CR}$ scattering for $\mathrm{GeV}$ particles (e.g., Zweibel 2013). There are even some sug- 
gestions from spectral signatures that only higher-energy $(\gtrsim 200 \mathrm{GeV})$ particles are affected by background ISM turbulence (Blasi et al. 2012).

Anisotropy in the distribution of CRs can destabilize Alfvén waves. Of particular interest and relevance is the cosmic-ray streaming instability (CRSI, Kulsrud \& Pearce 1969; Wentzel 1974; Skilling 1975a,b,c). Instability occurs when the bulk drift speed of the CRs exceeds the Alfvén speed in the background plasma. The instability drives the growth of Alfvén waves at the cost of free energy from CR anisotropy, and in the absence of wave damping the resulting scattering of particles off the waves will effectively reduce the CR drift speed to Alfvén speed. This process limits the bulk flow speed of the CRs, and simultaneously leads to energy and momentum exchange between the CRs and background gas. As a consequence, CRSI plays a fundamental role in the interaction between the CRs and ISM gas, and is the key element behind the picture of CR self-confinement as well as CR-driven winds (see, e.g., Zweibel 2013, 2017; Amato \& Blasi 2018 for reviews). On much larger scales in galaxy clusters, the CRSI may also be important in regulating heating (e.g. Guo \& Oh 2008; Enßlin et al. 2011; Fujita \& Ohira 2011; Wiener et al. 2013; Jacob \& Pfrommer 2017; Wiener et al. 2018; Wiener \& Zweibel 2018). Here, however, we shall focus on the parameter regime appropriate for cosmic ray interactions with the ISM.

Studies of CR interactions with the ISM on large scales, whether analytic or numerical, treat the CRs as fluid-like (e.g., Breitschwerdt et al. 1991; Breitschwerdt et al. 1993; Enßlin et al. 2007; Sharma et al. 2010; Pfrommer et al. 2017; Jiang \& Oh 2018; Thomas \& Pfrommer 2018), generally adopting the assumption of streaming, diffusion, or some combination. This is necessary given the huge scale separation between the macroscopic scale of astrophysical systems and the tiny gyro-scale of the CRs, but this approach is subject to the prescriptions adopted to treat the microphysical effects of CR diffusion and streaming.

In recent years, there has been a resurgence of interest in CR-driven galactic winds. While most numerical studies to date have adopted diffusion prescriptions (e.g. Jubelgas et al. 2008; Hanasz et al. 2013; Booth et al. 2013; Salem et al. 2014; Salem \& Bryan 2014; Simpson et al. 2016; Pakmor et al. 2016; Girichidis et al. 2016; Girichidis et al. 2018; Jacob et al. 2018), there have also been studies that compare wind outcomes when streaming vs. diffusive prescriptions are adopted for the CRs (Wiener et al. 2017; Ruszkowski et al. 2017). The dynamics of winds in which CRs stream at the Alfv'en speed have distinctive features, since the effective sound speed of gas increases as its density decreases, enabling steady-state CR-driven acceleration of cool gas to high velocities in typical galactic potentials (Mao \& Ostriker 2018). Most recently, several studies of wind driving have considered even more sophisticated treatment of the CR fluid, implementing prescriptions for wave damping and for decoupling of CRs from cold ISM components (Recchia et al. 2016; Holguin et al. 2018; Farber et al. 2018). While all of the above studies highlight the important role played by the $\mathrm{CRs}$, it is also evident that different microphysical prescriptions can yield dramatically different outcomes for wind properties. Currently, however, microphysical coefficients that are adopted in simulations are motivated on phenomenological grounds or based on classical analytic theory of the CRSI, diffusion, and wave damping. Development of a modern theoretical foundation for CR fluid treatments, with coefficients calibrated from numerical studies that directly follow the microphysics, will clearly aid progress in this field.

At microscopic level, our current understanding of the CRSI is limited to quasi-linear theory. We note that on the one hand, the instability can become non-linear when CR streaming is sufficiently strong, and eventually transitions to the Bell instability (Bell 2004) in more extreme environments such as super-Alfvénic shocks in supernova remnants. On the other hand, there are additional uncertainties related to mechanisms that lead to wave damping (see discussions in Section 7.2), many of which again require numerical studies.

Numerical studies of the CRSI at microphysical level per force must involve particle-in-cell (PIC) methods, and are inherently challenging because of the extreme ratios between the density of thermal and nonthermal particles and the scales relevant for the background thermal plasma compared to CR gyroradii. Another difficulty is that the weak level of CR anisotropy (of order a few times $v_{A} / c$ ) must be accurately represented, demanding a huge number of computational particles (see Section 3). Both of these issues make it very challenging for conventional particle-in-cell (PIC) methods to study the CRSI, especially in the regime relevant for the bulk of the ISM away from CR injection sites (but see Holcomb \& Spitkovsky 2018 and further discussion in Section 7.3).

Recently, we developed a new hybrid MHD-PIC method (Bai et al. 2015), implemented within the Athena code package (Stone et al. 2008). By treating the background thermal plasma as a fluid described by magnetohydrodynamics (MHD), we greatly alleviate the issue of scale separation between the thermal gas and CRs. Using this method, we are able to properly capture the basic properties of the CRSI, particularly in astrophysically relevant/realistic parameter regimes. For this purpose, in our first study we simplify by ignoring wave damping and concentrating on one-dimensional simulations that provide the highest possible resolution. By employing several novel techniques, we demonstrate that our method can accurately reproduce the linear growth of CSRI, and subsequently follow the quasi-linear diffusion of particles by scattering off of waves generated by the CRSI. We anticipate this effort will pave the way for future studies that incorporate additional physics under a variety of environments.

This paper is organized as follows. We describe the basic theory of the CRSI in Section 2. In Section 3, we present the techniques and methodology employed in our CRSI simulations using the MHD-PIC approach. Our simulation setup is described in Section 4. In Section 5, we present simulation results from two fiducial runs. This is followed by a parameter study in Section 6 . Our results are discussed in a broader context in Section 7, before we conclude in Section 8. Additional numerical aspects of 


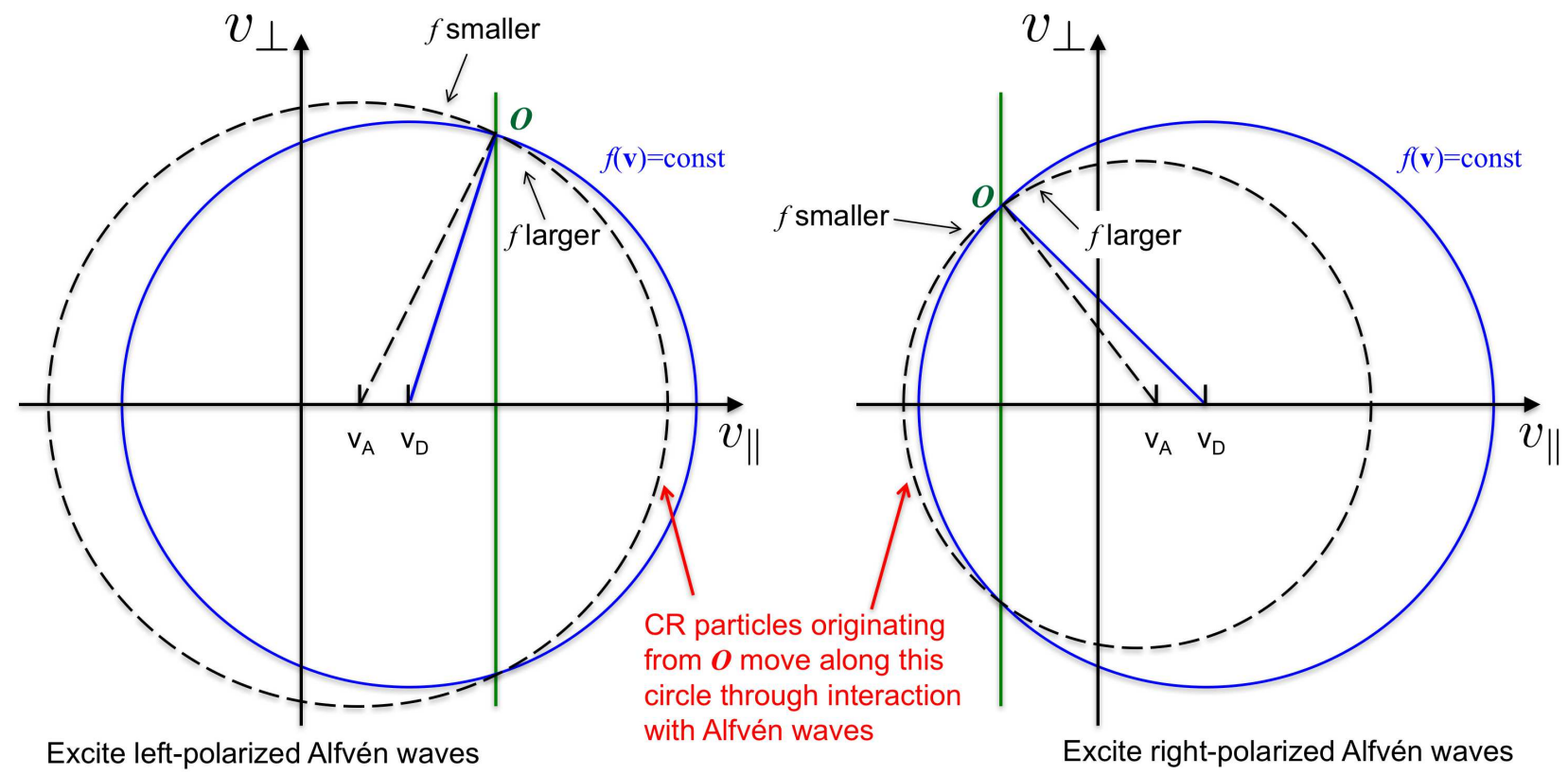

FIG. 1.- Schematic illustration of the basic physics of the CRSI. When the bulk CR drift velocity $v_{D}$ exceeds the Alfvén speed $v_{A}$, forward-traveling CR particles excite left-polarized, forward-propagating Alfvén waves, whereas backward-traveling CR particles excite right-polarized, forward-propagating Alfvén waves. We are taking particles located near a position $O$ in velocity space as an example. Also note the sizes of the blue and black circles should be comparable but has to be exaggerated in this schematic figure. See Section 2 for details.

our simulations are described in the Appendices.

\section{BASIC THEORY}

In this section, we briefly review the theory of the CRSI, which largely follows the description in Kulsrud (2005). We focus on the growth and saturation of the instability. While wave damping is another important ingredient for the CRSI, it will be addressed in our followup work and is not considered here.

\subsection{Physical Mechanism}

Without rigorous mathematical derivation, the basic physics of the CRSI can be understood schematically as illustrated in Figure 1. Consider a static background thermal plasma with constant density $\rho_{0}$ and constant magnetic field $\boldsymbol{B}=B_{0} \hat{x}$ with $B_{0}>0$. The Alfvén speed is thus $v_{A}=B_{0} / \sqrt{4 \pi \rho_{0}}$. Coexisting with the background plasma is a population of CRs. Let the initial CR velocity distribution be isotropic in a frame that drifts relative to the background gas at velocity $v_{D}$, with the drift velocity along the background field $\hat{x}$. Without loss of generality, let $v_{D}>0$ (along the direction of magnetic field). Let $f_{0}(\boldsymbol{v})$ or $f_{0}(\boldsymbol{p})$ denote the initial particle velocity or momentum distribution function, monotonically decreasing with increasing $p$. Note that individual CR particle speeds greatly exceed $v_{D}$, hence approximately half of the particles travel forward/backward with respect to the gas. For particles with mass $m$, charge $q$ and Lorentz factor $\gamma$, the cyclotron frequency $\Omega_{c}$ and gyro frequency $\Omega$ read

$$
\Omega_{c}=\frac{q B_{0}}{m c}, \quad \Omega=\Omega_{c} / \gamma
$$

The CR particles can experience gyro-resonance with circularly polarized Alfvén waves when the pattern of particle gyration matches that of the waves, and the frequency of the waves experienced by the particles matches their gyro-frequency. To satisfy the first condition, forward traveling CRs can resonate with left-handed, forward-propagating Alfvén waves, whereas backward traveling CRs can resonate with right-handed, forwardpropagating Alfvén waves (see Appendix A for our adopted conventions for left/right handedness). To satisfy the second condition, the parallel velocity of a particle $v_{\|}$must satisfy

$$
\omega-k v_{\|}= \pm \Omega \approx-k v_{\|},
$$

where $\omega$ and $k$ are the frequency and wave vector of the Alfvén wave (we only consider wave vector $k$ along the magnetic field), and the +/- sign corresponds to right/left polarized modes, respectively. Note that $\omega=$ $k v_{A}$, while typically the CR velocity (and its projection) $v_{\|} \gg v_{A}$. We can thus omit $\omega$ in the above equation. In other words, the CR particles travel so rapidly that Alfvén waves simply appear as a static pattern.

Now consider CR particles in velocity space decomposed into components parallel and perpendicular to the background magnetic field $\left(v_{\|}, v_{\perp}\right)$, and pick a representative particle located at point " $O$ " shown in Figure 1. To facilitate the illustration, we now assume particles are non-relativistic (but it can be easily generalized to relativistic CRs), and hence $f_{0}(\boldsymbol{v})$ is constant along a circle centered on $\left(v_{D}, 0\right)$ (the blue solid circle in Figure 1). Clearly, $f_{0}$ is larger (smaller) inside (outside) the circle. For linear Alfvén waves, their associated electric field vanishes in the wave frame. Because magnetic fields do no work on particles, each particle trajectory in velocity space must follow a circle centered in $\left(v_{A}, 0\right)$ (black dashed circle in Figure 1). Wave-particle inter- 
action generally leads to a quasi-linear diffusion (QLD) of particle pitch angle, towards removing the gradient of $f_{0}(\boldsymbol{v})$ across the dashed circle near point $O$. We see that when $v_{D}>v_{A}$, for forward traveling $\mathrm{CR}$ particles near $O$ (left panel), the net outcome should be that more particles with higher $v_{\|}$on the right hand side of point $O$ diffuse along the dashed circle towards smaller $v_{\|}$. In net, when $v_{D}>v_{A}$ the diffusion (via resonant interactions) of particles irreversibly gives momentum to the left-handed Alfvén waves, feeding their growth. Similarly, for backward traveling CR particles (right panel), more particles on the right side of $O$ diffuse along the dashed circle to the left side, with the change in $v_{\|}$feeding the growth of right-handed Alfvén waves. We can also easily see that if $v_{D}<v_{A}$, instead of wave excitation, the Alfvén waves will be damped.

This discussion illustrates how the CRSI is driven by the anisotropy of the CRs. At the same time, it suggests that CRSI growth is sustained by QLD of the CRs which produces secular and irreversible changes in the background plasma. This means that for a numerical method to capture linear growth of CRSI, it must properly capture QLD as well, which is an important fact to bear in mind when we discuss numerical methods in Section 3.

\subsection{Linear Instability of Waves}

We now proceed to a more quantitative description of the CRSI. Its derivation follows from standard procedures, as detailed in Kulsrud (2005). Here, we only summarize the necessary results needed for setting up and analyzing the simulations.

For convenience, in the discussions below and also throughout this paper, we assume that the mass of $\mathrm{CR}$ ions and background ions are identical (being $m$ ). As will be seen in Section 3.1, the value of $m$ has no physical significance and can always be absorbed into a factor for the charge-to-mass ratio. We thus drop it and express the momentum of and energy of individual CR particles as

$$
\boldsymbol{p}=\gamma \boldsymbol{v}, \quad E_{p}=\gamma c^{2},
$$

where $\boldsymbol{v}$ is a particle's velocity and $\gamma$ is its Lorentz factor. In other words, we effectively take $m=1$. However, we do retain the symbol $m$ in certain places for better clarity.

As defined earlier, we use $f_{0}(\boldsymbol{p})=f_{0}(p)$ to denote the initial CR distribution function in the frame moving at $v_{D}$, where the distribution is isotropic, with total CR number density given by $n_{\mathrm{CR}}=\int 4 \pi p^{2} f_{0}(p) d p$. For Alfvén waves with perturbations proportional to $\exp [\mathrm{i}(k x-\omega t)]$, the CR-modified dispersion relation reads

$$
\omega^{2}=k^{2} v_{A}^{2} \mp \frac{n_{\mathrm{CR}}}{n_{i}} \Omega_{c}\left(\omega-k v_{D}\right)\left[\left(1-Q_{1}\right) \pm \mathrm{i} Q_{2}\right],
$$

where $n_{i}$ is the density of background ions. The role of the CRs is reflected in the two dimensionless factors

$$
\begin{aligned}
Q_{1}(k) & \equiv \int d p \frac{4 \pi p^{2} f_{0}(p)}{n_{\mathrm{CR}}}\left(\frac{p_{\text {res }}}{2 p} \log \left|\frac{1+p / p_{\text {res }}}{1-p / p_{\text {res }}}\right|\right) \\
Q_{2}(k) & \equiv \frac{\pi}{2} \int_{p_{\text {res }}}^{\infty} d p \frac{4 \pi p^{2} f_{0}(p)}{n_{\mathrm{CR}}}\left(\frac{p_{\text {res }}}{p}\right)
\end{aligned}
$$

where the dependence of $Q_{1}$ and $Q_{2}$ on $k$ is reflected in the resonant momentum

$$
p_{\text {res }}(k)=\Omega_{c} / k,
$$

which is the minimum $\mathrm{CR}$ momentum to resonate with an Alfvén wave with wave number $k$ (at zero pitch angle). ${ }^{1}$

Physically, the term associated with 1 in the $\left(1-Q_{1}\right)$ factor is due to the background CR current along $\boldsymbol{B}_{0}$. The $Q_{1}$ term is from the non-resonant response of $\mathrm{CR}$ particles to the waves. It is straightforward to show that $Q_{1}$ approaches 1 in long-wavelength limit, and monotonically decreases to 0 in short wavelength limit. Thus, the real part of the $\mathrm{CR}$ contribution is bounded. The imaginary part, namely, the $Q_{2}$ term, results from the resonant response of $\mathrm{CR}$ particles to the waves. Note that at a given $k$, only particles with $p>p_{\text {res }}$ contribute to $Q_{2}$, as expected.

In the limit $n_{\mathrm{CR}} / n_{i} \ll 1$, the real part of the dispersion relation (4) is clearly dominated by the $k^{2} v_{A}^{2}$ term, and hence the term proportional to $\left(1-Q_{1}\right)$ can be neglected. In this case, the dispersion relation largely gives normal Alfvén waves, which can slowly grow or damp owing to the imaginary part of the dispersion relation (i.e., the $Q_{2}$ term from resonant response). This is the general CRSI originally derived (Kulsrud \& Pearce 1969), where the forward propagating modes, both left and right handed, grow at the same rates, given by

$$
\Gamma(k)=\frac{1}{2} \frac{n_{\mathrm{CR}}}{n_{i}} \Omega_{c}\left(\frac{v_{D}}{v_{A}}-1\right) Q_{2}(k),
$$

and hence growing modes are essentially linearly polarized. Clearly, instability occurs only when $v_{D}>v_{A}$. Moreover, the maximum growth rate is of the order $\left(n_{\mathrm{CR}} / n_{i}\right) \Omega_{c}$ at wavelengths that maximizes $Q_{2}$ (to reach order unity), which typically correspond to the resonant scale with lowest energy CRs. Backward propagating modes are damped.

More specifically, the initial distribution function is commonly considered to be a power law with

$$
f_{0}(p) \propto p^{-4+\alpha}, \quad p_{0} \leq p \leq p_{\max }
$$

and $f_{0}(p)=0$ otherwise. Fermi acceleration gives $\alpha=0$ (e.g., Bell 1978; Blandford \& Ostriker 1978). In typical ISM conditions, the Galactic CR population corresponds to $\alpha \approx 0.7$ for $\mathrm{CR}$ particle energy beyond $\sim \mathrm{GeV}$, and $n_{\mathrm{CR}} / n_{i} \sim 10^{-9}$. The $Q_{2}$ factor is given by

$$
Q_{2}(k)=\frac{\pi}{2}\left(\frac{1+\alpha}{2+\alpha}\right) p_{\mathrm{res}} \frac{p_{\min }^{-(2+\alpha)}-p_{\max }^{-(2+\alpha)}}{p_{0}^{-(1+\alpha)}-p_{\max }^{-(1+\alpha)}},
$$

where $p_{\min }=\operatorname{Min}\left[\operatorname{Max}\left(p_{\text {res }}, p_{0}\right), p_{\max }\right]$. The maximum

1 The dispersion relation shown here is equivalent to Equations (11)-(13) in Amato \& Blasi (2009) with slight differences in sign conventions. The terms $Q_{1}$ and $Q_{2}$ arise from integrals containing $d f / d p$ (see also Equation (69) in Chapter 12 of Kulsrud (2005)); this is the mathematical embodiment of the situation depicted in Figure 1, in which a gradient in $f$ leads to wave growth. We have performed integration by parts to simplify the results, and $d f / d p$ no longer appears. 


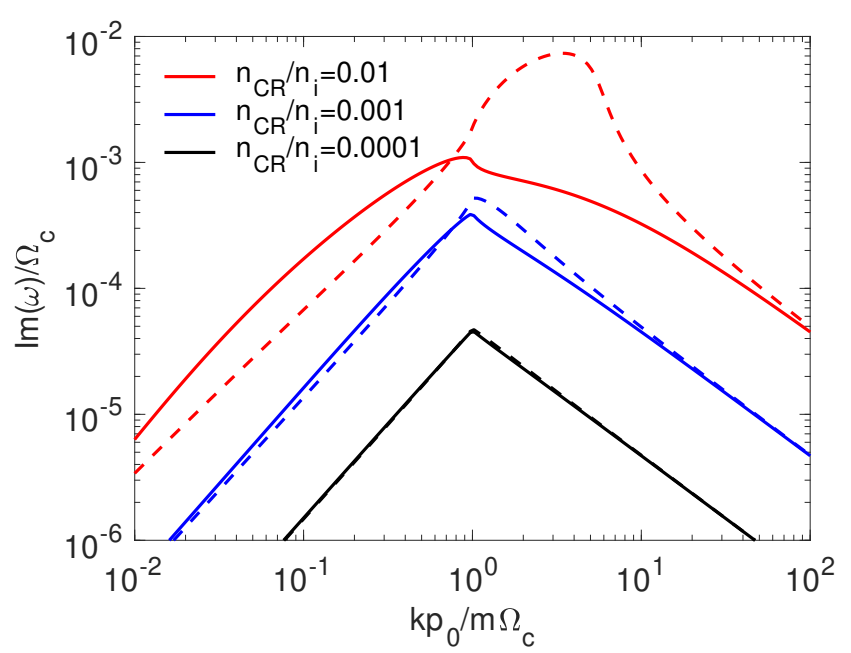

FIG. 2.- Linear dispersion relation of the CRSI with a truncated power-law $\mathrm{CR}$ distribution function (8), with fiducial parameters $v_{D}=2 v_{A}, p_{0} / m=300 v_{A}, s=4.5$. Three values of $n_{\mathrm{CR}} / n_{i}$ are adopted, as shown in the red $\left(10^{-2}\right)$, blue $\left(10^{-3}\right)$ and black $\left(10^{-4}\right)$ lines. The growth rates for left (solid) and right (dashed) handed modes are shown separately. Note the gradual development and dominance of the non-resonant Bell mode at $n_{\mathrm{CR}} / n_{i} \gtrsim 10^{-3}$. We focus on the regime with smaller $n_{\mathrm{CR}} / n_{i}$, being more realistic in the bulk of the ISM.

growth rate is achieved at $p_{\text {res }}=p_{0}$, or

$$
k=k_{0} \equiv \Omega_{c} / p_{0} \text {. }
$$

This corresponds to the resonant wavelength for particles with $p=p_{0}$ (and zero pitch angle). In the limit $p_{\max } \rightarrow$ $\infty$, we have

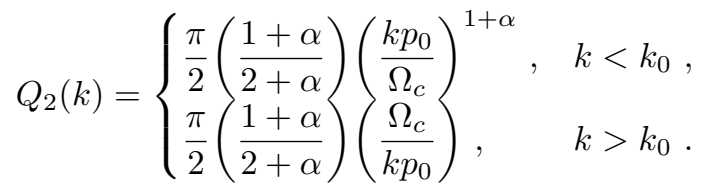

Taking into account the term from $\left(1-Q_{1}\right)$ breaks the degeneracy between left and right handed modes, especially when $n_{\mathrm{CR}} / n_{i}$ increases. In particular, it makes the right-handed modes grow faster and more dominant towards short wavelength (see Figure 2), and eventually, it smoothly transitions to the non-resonant Bell mode at $k \gtrsim k_{0}$ (Bell 2004). This transition occurs when (Amato \& Blasi 2009)

$$
\frac{n_{\mathrm{CR}}}{n_{i}} \gtrsim \frac{v_{A}^{2}}{p_{0} v_{D}} .
$$

For simulations in this work, we fiducially adopt $v_{D} / v_{A}=2, p_{0} / v_{A}=300, \alpha=0.5$, and $n_{\mathrm{CR}} / n_{i}=10^{-4}$ to $10^{-3}$. The resulting dispersion relation is shown in Figure 2, which displays all features discussed above. Note that for application to realistic large-scale ISM conditions, a very low value of low $n_{\mathrm{CR}} / n_{i} \sim 10^{-9}$ is appropriate, such that the left/right handed modes are degenerate. However, in practice, the values of $n_{\mathrm{CR}} / n_{i}$ we use in the simulations have to be much higher in order to follow wave growth and particle quasi-linear diffusion within a reasonable computational time. We see that for our fiducial parameters, the transition to non-resonant modes occurs when $n_{\mathrm{CR}} / n_{i} \gtrsim 10^{-3}$.

\subsection{Quasi-Linear Diffusion of Particles}

The growth of the CRSI creates a spectrum of Alfvén waves. The intensity of the waves at wave number $k$ is described by $I(k)$, so that

$$
\int I(k) d k=\left\langle\frac{\delta B^{2}}{B_{0}^{2}}\right\rangle,
$$

where angle bracket represents spatial averages. In Appendix A, we describe the procedures to decompose parallel propagating Alfvén waves into different modes (left/right handed, forward/backward propagation), from which we obtain $I(k)$ for each mode.

In a spatially uniform media (as in our simulations), quasilinear diffusion (QLD) leads to pitch angle scattering in the frame of the Alfvén waves, with pitch angle $\theta$ more commonly replaced by $\mu \equiv \cos \theta$. We use subscript ' $w$ ' to denote quantities measured in this frame. The evolution of $f_{w}$, the CR distribution function in the wave frame in a homogeneous system, is described by (e.g., Jokipii 1966; Kulsrud \& Pearce 1969)

$$
\frac{\partial f_{w}}{\partial t}=\frac{\partial}{\partial \mu_{w}}\left[\frac{1-\mu_{w}^{2}}{2} \nu\left(\mu_{w}\right) \frac{\partial f_{w}}{\partial \mu_{w}}\right]
$$

where

$$
\nu\left(\mu_{w}\right)=\pi \Omega k_{\mathrm{res}} I\left(k_{\mathrm{res}}\right)
$$

and

$$
k_{\mathrm{res}} \equiv \Omega_{c} /\left(p_{w} \mu_{w}\right)
$$

is the wave number of the resonant waves. $^{2}$

Our simulations are conducted in the frame where the initial $\mathrm{CR}$ distribution is isotropic, whose relative velocity to the wave frame is $\Delta v \equiv v_{D}-v_{A}$. Using subscripts '\|' and ' $\perp$ ' to denote components parallel and perpendicular to background field $\boldsymbol{B}_{0}$, and to first order of $\Delta v \ll c$, we have for particle momenta

$$
p_{w, \perp}=p_{\perp}, \quad p_{w, \|}=p_{\|}+\gamma \Delta v .
$$

With conservation of phase-space volume, it follows that the distribution functions $f=f_{w}$. The conversion relations between the two frames read

$$
p_{w}=p\left(1+\mu \frac{\Delta v}{v}\right), \quad \mu_{w}=\mu+\left(1-\mu^{2}\right) \frac{\Delta v}{v},
$$

where $v \sim c$ is the velocity corresponding to momentum $p$. In the quasi-linear regime, the distribution function only deviates slightly from $f_{0}$, and hence we can write $f(p, \mu)=f_{0}(p)+\delta f(p, \mu)$. Transforming to the wave frame, and to the leading order of $\delta f / f_{0}$ and $\Delta v / c$, we obtain

$$
\begin{aligned}
& f_{w}\left(p_{w}, \mu_{w}\right)=f(p, \mu) \\
\approx & f_{0}\left(p_{w}\right)+\delta f\left(p_{w}, \mu_{w}\right)-\mu_{w} \frac{\partial f_{0}}{\partial \ln p} \frac{\Delta v}{v_{w}} .
\end{aligned}
$$

${ }^{2}$ In calculating $\nu$ from Equation (15), we use $I\left(\left|k_{\text {res }}\right|\right)$ and choose the intensity for left/right handed waves for positive/negative $\mu_{w}$. Also note our normalization of $I(k)$ only accounts for the magnetic energy spectrum (will be doubled for total energy spectrum). Because of these normalization choices, our expression for $\nu$ does not have a factor $1 / 4$. 
In the wave frame, we have

$$
\frac{\partial f_{w}}{\partial \mu_{w}} \approx \frac{\partial \delta f}{\partial \mu_{w}}-\frac{\partial f_{0}}{\partial \ln p} \frac{\Delta v}{v_{w}} .
$$

In the fully saturated state, we expect $\partial f_{w} / \partial \mu_{w}=0$, and hence the pitch angle distribution should satisfy

$$
\frac{\partial \delta f_{\mathrm{sat}}}{\partial \mu_{w}} \approx \frac{\partial f_{0}}{\partial \ln p} \frac{\Delta v}{v} \Rightarrow \delta f_{\mathrm{sat}} \approx \mu_{w} \frac{\partial f_{0}}{\partial \ln p} \frac{\Delta v}{v} .
$$

The amplitude of the Alfvén waves in the saturated state can also be estimated. The net momentum density acquired by the CRs after saturation is reached is

$$
\Delta \mathcal{P}_{\mathrm{CR}}=\int \mu p\left(\delta f_{\mathrm{sat}}\right) d^{3} p=\frac{4 \pi}{3} \int \frac{\partial f_{0}}{\partial \ln p} \frac{\Delta v}{v} p^{3} d p .
$$

Note that with $\partial f_{0} / \partial p<0$, this implies that if initially $\Delta v=v_{D}-v_{A}>0$, in saturation the $\mathrm{CR}$ distribution must have acquired a momentum deficit, $\Delta \mathcal{P}_{\mathrm{CR}}<0$. In the limit that all CRs are relativistic, this yields

$$
\Delta \mathcal{P}_{\mathrm{CR}} \approx-\frac{4}{3} \frac{n_{\mathrm{CR}} \Delta v}{c}\langle p\rangle
$$

where $\langle p\rangle \equiv \int f_{0} p d^{3} p / \int f_{0} d^{3} p$. For the non-relativistic $\mathrm{CR}$ limit, the momentum reduction is $\Delta \mathcal{P}_{\mathrm{CR}} \approx$ $-m n_{\mathrm{CR}} \Delta v$.

The deficit in CR momentum must have been transferred to forward-propagating Alfvén waves. Note that there is an equipartition in kinetic and magnetic energies in Alfvén waves, with effective momentum density in waves (the Poynting flux divided by $v_{A}^{2}$; cf. Kulsrud 2005) given by

$$
\mathcal{P}_{\text {wave }}=\rho v_{A}\left\langle\frac{\delta B^{2}}{B_{0}^{2}}\right\rangle .
$$

Following the discussion in Kulsrud (see also Wentzel 1974), this momentum density represents the growth of Maxwell and Reynolds stresses in waves needed to transfer the CR momentum to the bulk gas, and is exhibited as a small fractional change in the momentum of the bulk gas.

Equating $\left|\Delta \mathcal{P}_{\mathrm{CR}}\right|$ with $\mathcal{P}_{\text {wave, }}$, we can obtain the expected saturation level for magnetic fluctuations. For ultra-relativistic and non-relativistic cases, we simply have

$$
\frac{\delta B^{2}}{B_{0}^{2}} \approx \frac{4}{3} \frac{\langle p\rangle}{m c} \frac{n_{\mathrm{CR}}}{n_{i}} \frac{\Delta v}{v_{A}}, \quad \text { or } \quad \approx \frac{n_{\mathrm{CR}}}{n_{i}} \frac{\Delta v}{v_{A}} .
$$

The real situation (as we consider) is of course somewhere in between nonrelativistic and ultrarelativistic, but the dependence of wave amplitude on the basic problem parameters is clear. These relations show that the saturation level of the instability is limited by the initial free energy in CR streaming. The above upper limit for magnetic field amplitudes would be reduced in the presence of damping.

The timescale for the CRs to relax to a state of isotropy in the wave frame is characterized by the scattering frequency $\nu$, which depends on both the pitch angle and the wave amplitudes. This means that scattering across different pitch angle ranges can take different amounts of time. Still, one may define a characteristic scattering frequency for the bulk CR population as

$$
\nu_{\mathrm{QLD}} \sim \nu(p, \mu) \sim \Omega \frac{\delta B^{2}}{B_{0}^{2}} .
$$

Note that wave growth is eventually quenched as the gradient in distribution function is smeared out through QLD. This suggests that another way to estimate the wave saturation amplitude is by equating the above $\nu_{\mathrm{QLD}}$ with $\Gamma$ from Equation (7), which is indeed similar (to order-of-magnitude) to the more rigorous estimate discussed above.

\subsection{Reflection Across $90^{\circ}$ Pitch Angle}

In Equation (16), when $\mu_{m} \rightarrow 0$ (i.e., pitch angle approaches $\left.90^{\circ}\right), k_{\text {res }} \rightarrow \infty$, i.e., the resonant wavelength becomes infinitely small. There is little energy in the waves generated from the CRSI towards the shortest wavelength, leading to a bottleneck for particles to be scattered across this $90^{\circ}$ pitch angle. This is a wellknown problem, and without crossing the $90^{\circ}$ pitch angle, the CRSI would saturate prematurely far from fully utilizing the free-energy in the system.

This problem has been discussed extensively in the literature. Within quasi-linear theory, relaxing the magnetostatic approximation [Equation (2)] allows for additional resonant interactions when both forward and backward propagating waves are present (Schlickeiser 1989), covering a pitch angle of $\Delta \theta \sim 2 v_{A} / c$ around $90^{\circ}$.

Magnetic mirroring is more commonly invoked in overcoming the $90^{\circ}$ problem (e.g., Felice \& Kulsrud 2001). With magnetic moment $M \equiv p_{\perp}^{2} / 2 B$ being an adiabatic invariant, a particle will experience a backward mirror force when its guiding center travels along a positive gradient in total field strength. For particle pitch angle sufficiently close to $90^{\circ}$, this mirror force can lead to reflection, thus directly jumping over the $90^{\circ}$ barrier. With total field strength $B=\sqrt{B_{0}^{2}+\delta B^{2}} \approx B_{0}\left(1+\delta B^{2} / 2 B_{0}^{2}\right)$, the critical pitch angle cosine below which mirror reflection can occur is thus given by

$$
\mu_{\mathrm{mir}} \approx \frac{1}{\sqrt{2}} \frac{\delta B}{B_{0}} .
$$

One thus expects mirror reflection to take over when QLD manages to scatter particles to pitch angle $\mu \sim$ $\mu_{\text {mir. }}$.

Note that mirror reflection requires slow changes in field strength over many particle gyro orbits. Given that a broad range of wave spectrum is excited by the CRSI, including those at small scales $\left(\lesssim k_{\text {res }}^{-1}\right)$ for particles of a given momentum, we can also imagine the opposite situation, where changes in field strength occurs more abruptly. For instance, an abrupt change of perpendicular field by $\delta B_{d i s}$ would change the direction of particle motion by $\delta B_{d i s} / B_{0}$, and in the mean time, the change the particle pitch angle is also on the order of $\sim \delta B_{\text {dis }} / B_{0}$. Depending on the gyro-phase, particles have a good chance to be reflected when $\mu \lesssim \delta B_{\text {dis }} / B_{0}$.

The (extreme) scenario outlined above falls into the category of non-linear wave-particle interaction: consid- 
ering particle orbits in perturbed fields (rather than unperturbed fields as in quasi-linear theory) gives resonance broadening (e.g., Dupree 1966; Völk 1973; Achterberg 1981 ), and alleviates the $90^{\circ}$ problem. It is more effective towards larger wave amplitudes, and eventually enters the regime of strong MHD turbulence (Yan \& Lazarian 2008). As we will show, our analysis suggests that this effect is primarily responsible for overcoming the $90^{\circ}$ barrier; for more discussion see Section 5.4.

\section{NUMERICAL METHOD}

There are several challenges that confront numerical study of the CRSI. First, there is substantial physical scale separation, which is exhibited as follows.

- The separation between microscopic scales of the background plasma, including the electron and ion skin depths $\left(c / \omega_{p e}\right.$ or $c / \omega_{p i}$ that full-PIC or hybridPIC simulations must resolve, where $\omega_{p e}$ and $\omega_{p i}$ are the electron and ion plasma frequencies), and the resonant scale of the CRSI ( $\sim$ gyro-radii of lowest-energy, trans-relativistic CRs). The ratio of the two scales is at least of the order $c / v_{A}$, which is prohibitively large for conventional PIC simulations if one were to approach realistic conditions.

- For quasi-linear diffusion of particles, individual CR particles must encounter a sufficient number of independent wave packets, so as to experience random-walk behavior in pitch angle evolution.

- The issue with particles scattering across the $90^{\circ}$ pitch angle. Particles with pitch angle near $90^{\circ}$ have resonant wavelength much shorter than their gyro-radii, implying that the most unstable wavelength of the CRSI must be very finely resolved.

The MHD-PIC approach uniquely alleviates the first scale separation issue by bypassing the microscopic plasma scales (ion skin depth) of the background thermal plasma. However, the remaining two issues remain challenging. Clearly, very high resolution is needed to capture scattering across $90^{\circ}$ pitch angle. In the mean time, when employing periodic boundary conditions, a sufficiently long simulation domain that cover many resonant scales is essential so that particles do not experience the same wave packets upon re-entering the domain. This issue will be discussed further in Section 3.4 and Appendix C.

In addition to the above challenges of physical scale separation, another challenge in modeling the regime of interest for the large-scale ISM is the huge ratio between the number density of $\mathrm{CR}$ particles and number density of background ISM particles, $n_{\mathrm{CR}} / n_{i} \sim 10^{-9}$. Cosmic ray and ISM pressures, however, are generally within an order of magnitude of each other. For modeling the largescale ISM with its extremely small $n_{\mathrm{CR}} / n_{i}$, the MHDPIC method is advantageous in comparison to full- or hybrid-PIC in that there there is no need to represent the background plasma with individual particles.

The third major challenge is that capturing the resonant condition still requires very large number of $\mathrm{CR}$ particles. For a given resonant mode, particles of a given momentum can (if capable) contribute only at a specific pitch angle. Correspondingly, only a tiny fraction of particles can potentially be in resonance with a given MHD wave mode. This issue may be seen from another viewpoint. The level of anisotropy in the CR distribution function is of the order $v_{D} / c \ll 1$. Such weak level of anisotropy must be accurately represented by the angular distribution of particles, again demanding for a huge number of particles in each cell.

Even though the MHD-PIC method is able to concentrate all particles in the CRs, properly capturing CRSI is still challenging when $v_{D} / c \ll 1$. To overcome this issue, we employ the $\delta f$ method, which dramatically improves the signal-to-noise and allows us to successfully simulate the CRSI with substantially reduced number of particles (see Section 3.2 and Appendix B). Below, we describe our methodology in detail.

\subsection{Formulation}

We solve CR-modified MHD equations in conservative form $^{3}$

$$
\frac{\partial \rho}{\partial t}+\nabla \cdot\left(\rho \boldsymbol{v}_{g}\right)=0
$$

$$
\begin{aligned}
\frac{\partial\left(\rho \boldsymbol{v}_{g}\right)}{\partial t}+\nabla & \cdot\left(\rho \boldsymbol{v}_{g}^{T} \boldsymbol{v}_{g}-\boldsymbol{B}^{T} \boldsymbol{B}+P^{*} \mathrm{I}\right) \\
& =-\left(q_{\mathrm{CR}} n_{\mathrm{CR}} \mathcal{E}+\frac{\boldsymbol{J}_{\mathrm{CR}}}{c} \times \boldsymbol{B}\right),
\end{aligned}
$$

$$
\frac{\partial E}{\partial t}+\nabla \cdot\left[\left(E+P^{*}\right) \boldsymbol{v}_{g}-\left(\boldsymbol{B} \cdot \boldsymbol{v}_{g}\right) \boldsymbol{B}\right]=-\boldsymbol{J}_{\mathrm{CR}} \cdot \mathcal{E}
$$

where $P^{*} \equiv P_{g}+B^{2} / 2, \mathcal{E} \equiv-\boldsymbol{v}_{g} \times \boldsymbol{B} / c$ is the electric field, I is the identity tensor, and the total energy density of the gas is defined as

$$
E=\frac{P_{g}}{\Gamma-1}+\frac{1}{2} \rho v_{g}^{2}+\frac{1}{2} B^{2} .
$$

In the above, $\rho, \boldsymbol{v}_{g}, P_{g}$ are gas density, velocity, and pressure, and $\Gamma$ is the adiabatic index. Note we have adopted the units where magnetic permeability is unity so that factors of $(4 \pi)^{-1 / 2}$ that would otherwise appear with the magnetic field are eliminated.

The CR number density $n_{\mathrm{CR}}$ and current density $\boldsymbol{J}_{\mathrm{CR}}$ are defined as

$$
\begin{aligned}
& n_{\mathrm{CR}}(t, \boldsymbol{x})=\int f(t, \boldsymbol{x}, \boldsymbol{p}) d^{3} \boldsymbol{p}, \\
& \boldsymbol{J}_{\mathrm{CR}}(t, \boldsymbol{x})=q_{\mathrm{CR}} \int \boldsymbol{v} f(t, \boldsymbol{x}, \boldsymbol{p}) d^{3} \boldsymbol{p},
\end{aligned}
$$

where $q_{\mathrm{CR}}$ is individual CR particle charge, and $\boldsymbol{p}, \boldsymbol{v}$ are the momentum and velocity of individual CR particles, with $f(t, \boldsymbol{x}, \boldsymbol{p})$ is the local CR momentum distribution function.

3 These equations are simplified from those derived in Bai et al. (2015) in that terms related to the CR-modified Hall effect are dropped. As discussed there, these terms are important only when $\left(n_{\mathrm{CR}} v_{D}\right) /\left(n v_{A}\right) \gtrsim 1$, corresponding to very strong CR streaming. In our simulations, this ratio is $\lesssim 10^{-3}$, and in reality the ratio is several orders of magnitude lower. 
While the MHD formulation is non-relativistic, the CR particles can be relativistic. We define an artificial speed of light $\mathbb{C}$ for the $\mathrm{CR}$ particles, and the overall formulation is consistent as long as $\mathbb{C} \gg v_{A}$ (typical MHD velocities). For an individual particle $j$, we have $\boldsymbol{p}_{j}=\gamma_{j} \boldsymbol{v}_{j}$, with Lorentz factor given by

$$
\gamma_{j}=\frac{\sqrt{\mathbb{C}^{2}+p_{j}^{2}}}{\mathbb{C}}=\frac{\mathbb{C}}{\sqrt{\mathbb{C}^{2}-v_{j}^{2}}} .
$$

The particle equation of motion reads

$$
\frac{d \boldsymbol{p}_{j}}{d t}=\left(\frac{q}{m c}\right)_{j}\left(c \mathcal{E}+\boldsymbol{v}_{j} \times \boldsymbol{B}\right) .
$$

Note that as mentioned earlier, we have dropped the individual particle mass in the definition of particle momentum. This mass is absorbed to the factor $q /(m c)$, representing particle charge-to-mass ratio. Also note that the physical speed of light $c$ has no significance in all equations where it appears: inspection of Equations (29), (30), (32), and (34) shows that $c$ appears only in the combinations $J_{\mathrm{CR}} / c, c \mathcal{E}$, and $q / m c$, and for numerical purposes can be absorbed into the parameter $(q / m c)$.

In our simulations, ideal MHD equations for the background thermal plasma are solved using the Athena MHD code (Stone et al. 2008), which is a higher-order Godunov code with constrained transport to enforce the divergence-free condition of the magnetic field. The corner transport upwind (CTU, Gardiner \& Stone 2005, 2008) method is adopted for time integration. We use the Roe Riemann solver (Roe 1981) and third-order reconstruction in characteristic variables. CR particles are implemented as Lagrangian particles, and the coupling between CRs and the background plasma is handled by adding source terms in the gas momentum and energy updates, as indicated on the right hand sides of Equations (29), (30). The CR particle equation of motion (34) is solved by the standard Boris integrator (Boris 1970). A standard triangular-shaped cloud (TSC) scheme (Birdsall \& Langdon 2005) is used for interpolating grid quantities to particle locations, and for depositing particle quantities back to the grid (e.g., to evaluate $n_{\mathrm{CR}}$ and $\boldsymbol{J}_{\mathrm{CR}}$ in Equation (32)). Details of the CR implementation are described in Bai et al. (2015).

\subsection{The $\delta f$ Method}

The original CR implementation in Bai et al. (2015) interprets individual particles as representing the full distribution function $f(\boldsymbol{x}, \boldsymbol{p})$, and evaluates physical quantities such as $n_{\mathrm{CR}}, \boldsymbol{J}_{\mathrm{CR}}$ directly according to Equation (32). We refer to this approach as the "full- $f$ method", which can be subject to large Poisson noise.

In the case where the distribution function is close to some equilibrium distribution $f_{0}(\boldsymbol{x}, \boldsymbol{p})$, one can use the fact that $f_{0}$ is already known analytically, and employ individual particles as Lagrangian markers taken to represent the difference, $\delta f$, between $f_{0}$ and the full distribution function $f$. This is known as the $\delta f$ method (e.g., Parker \& Lee 1993; Dimits \& Lee 1993; Hu \& Krommes 1994; Denton \& Kotschenreuther 1995; Kunz et al. 2014).
The basis of the $\delta f$ method is the Liouville theorem, which requires that the full distribution function $f$ be constant along particle trajectories in phase space (i.e., characteristics).

To implement the $\delta f$ method, we first record the initial value of $f$ at $t=0$ for all particles (which is essentially $\left.f_{0}\right)$. Then at every time $t$, we assign a weight $w_{j}$ to each particle $j$ given by

$$
w_{j} \equiv \frac{\delta f\left(t, \boldsymbol{x}_{j}(t), \boldsymbol{p}_{j}(t)\right)}{f\left(t, \boldsymbol{x}_{j}(t), \boldsymbol{p}_{j}(t)\right)}=1-\frac{f_{0}\left(\boldsymbol{x}_{j}(t), \boldsymbol{p}_{j}(t)\right)}{f\left(0, \boldsymbol{x}_{j}(0), \boldsymbol{p}_{j}(0)\right)} .
$$

Physical quantities such as the CR number density and current density are obtained by

$$
\begin{array}{r}
n_{\mathrm{CR}}(t, \boldsymbol{x})=n_{\mathrm{CR}, 0}+\int \delta f(t, \boldsymbol{x}, \boldsymbol{p}) d^{3} \boldsymbol{p} \\
\simeq n_{\mathrm{CR}, 0}+\sum_{j=1}^{N_{p}} w_{j} S\left(\boldsymbol{x}-\boldsymbol{x}_{j}\right) \\
\boldsymbol{J}_{\mathrm{CR}}(t, \boldsymbol{x})=\boldsymbol{J}_{\mathrm{CR}, 0}+q_{\mathrm{CR}} \int \boldsymbol{v} \delta f(t, \boldsymbol{x}, \boldsymbol{p}) d^{3} \boldsymbol{p} \\
\simeq \boldsymbol{J}_{\mathrm{CR}, 0}+q_{\mathrm{CR}} \sum_{j=1}^{N_{p}} w_{j} \boldsymbol{v}_{j} S\left(\boldsymbol{x}-\boldsymbol{x}_{j}\right)
\end{array}
$$

where $S(\boldsymbol{r})$ is the shaping function used in particle interpolation (i.e., the TSC scheme), and $n_{\mathrm{CR}, 0}$ and $\boldsymbol{J}_{\mathrm{CR}, 0}$ are obtained analytically from $f_{0}$. By contrast, full- $f$ method corresponds to setting $w_{j}=1, n_{\mathrm{CR}, 0}=0$ and $\boldsymbol{J}_{\mathrm{CR}, 0}=0$. In this way, the $\delta f$ method dramatically reduces the Poisson noise of the background particle distribution, allowing the signal from the $\delta f$ part to be substantially boosted.

In practice, we have found that the $\delta f$ method is essential. Without employing it (i.e., using the full- $f$ method), and using our fiducial simulation parameters, we barely observe the development of the CRSI even using $\sim 10^{4}$ particles per cell, and the system is almost entirely dominated by Poisson noise (see Appendix D for more information).

We note that with the $\delta f$ method, exact conservation of the total (gas and CR) momentum and energy, achieved in the full- $f$ method, is lost. This is inevitable, since the formulation of the $\delta f$ method is intrinsically non-conservative. However, the benefit from low noise is of overwhelming importance for the CRSI problem, and in practice we find that the error in total momentum and energy is negligible throughout all our simulations.

\subsection{The $\kappa$ distribution}

While the CRSI is commonly analyzed with $f_{0}$ being a simple truncated power law (8), this is incompatible with the $\delta f$ method. The reason is that the $\delta f$ method requires $f_{0}$ to be finite at all $p$ since it serves as the normalization factor in the weighting function, Equation (35). To avoid divergence, we modify $f_{0}$ to be a $\kappa$ distribution (e.g., see Summers \& Thorne 1991 and references 
TABLE 1

List OF MAIN SIMULATION RUNS

\begin{tabular}{c|cc|cccc|c}
\hline \hline Run & $v_{D} / v_{A}$ & $n_{\mathrm{CR}} / n_{i}$ & $\begin{array}{c}\text { Domain size } \\
L_{x}\left(d_{i}\right)\end{array}$ & $\begin{array}{c}\text { Domain size } \\
L_{x} / \lambda_{m}\end{array}$ & $\begin{array}{c}\text { resolution } \\
\left(d_{i} \text { per cell }\right)\end{array}$ & $\begin{array}{c}N_{p} \text { per bin } \\
(\text { per cell })\end{array}$ & $\begin{array}{c}\text { Runtime } \\
\left(\Omega_{c}^{-1}\right)\end{array}$ \\
\hline Fid & 2.0 & $1.0 \times 10^{-4}$ & $9.6 \times 10^{4}$ & 51 & 10 & 256 & $10^{6}$ \\
M3 & 2.0 & $1.0 \times 10^{-3}$ & $9.6 \times 10^{4}$ & 51 & 10 & 256 & $10^{6}$ \\
M5 & 2.0 & $1.0 \times 10^{-5}$ & $3.2 \times 10^{4}$ & 17 & 10 & 256 & $1.5 \times 10^{6}$ \\
vD4 & 4.0 & $1.0 \times 10^{-4}$ & $9.6 \times 10^{4}$ & 51 & 10 & 256 & $10^{6}$ \\
vD8 & 8.0 & $1.0 \times 10^{-4}$ & $9.6 \times 10^{4}$ & 51 & 10 & 256 & $10^{6}$ \\
Fid-Hires & 2.0 & $1.0 \times 10^{-4}$ & $6.4 \times 10^{4}$ & 34 & 4 & 256 & $10^{6}$ \\
Fid-Short & 2.0 & $1.0 \times 10^{-4}$ & $3.2 \times 10^{4}$ & 17 & 10 & 256 & $10^{6}$ \\
Fid-Np64 & 2.0 & $1.0 \times 10^{-4}$ & $9.6 \times 10^{4}$ & 51 & 10 & 64 & $10^{6}$ \\
Fid-Np1024 & 2.0 & $1.0 \times 10^{-4}$ & $9.6 \times 10^{4}$ & 51 & 10 & 1024 & $2 \times 10^{5}$ \\
\hline \hline
\end{tabular}

Fixed parameters: $\mathbb{C} / v_{A}=300, p_{0} / m v_{A}=300, \kappa=1.25$, and initial wave amplitude $A=10^{-4}$. Note the most unstable wavelength is $\lambda_{m}=2 \pi p_{0} /\left(m \Omega_{c}\right) \approx 1885 d_{i}$ for all models.
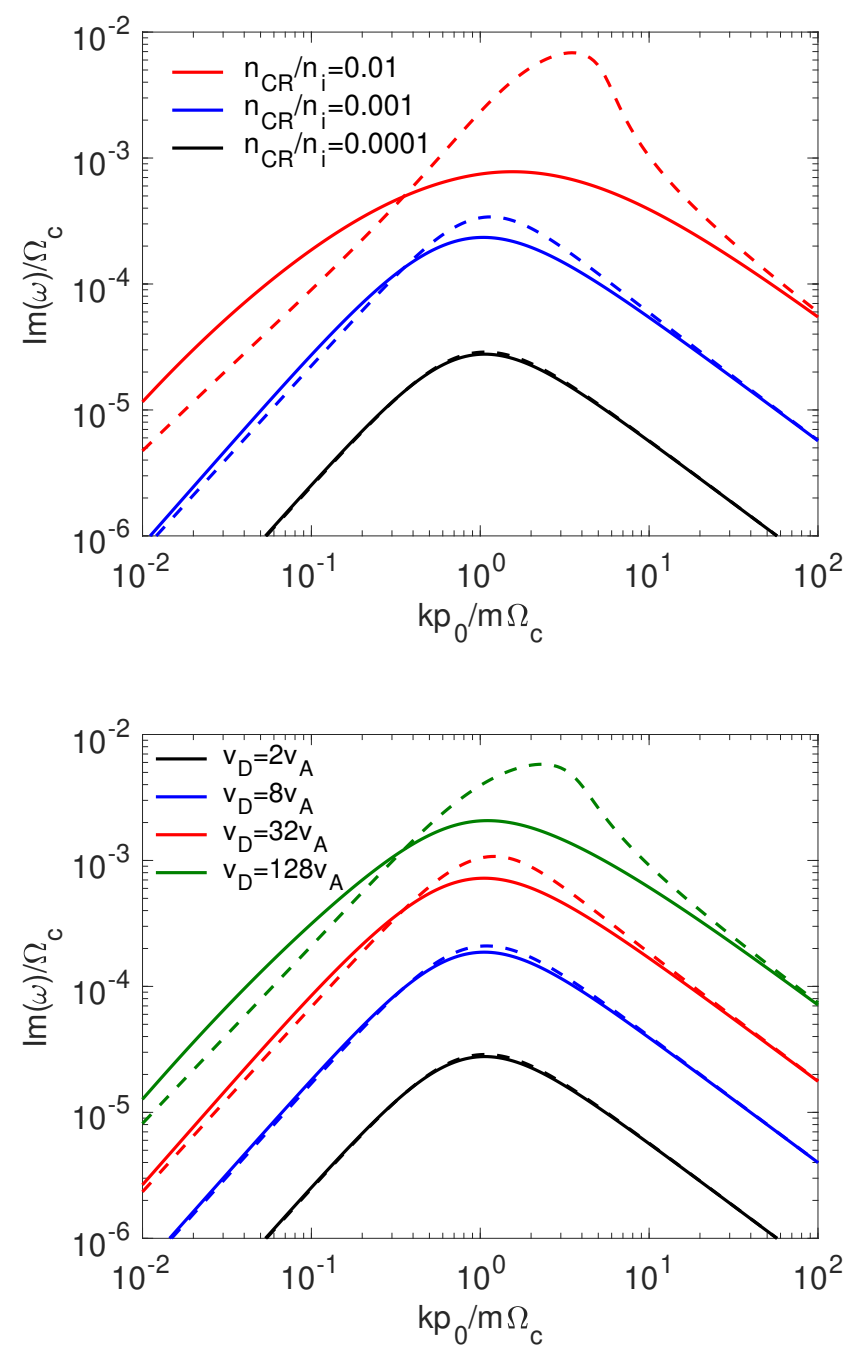

FIG. 3.- Linear dispersion relation of the CRSI for a $\kappa$ distribution (38) with $\kappa=1.25$, with fiducial parameters $n_{\mathrm{CR}} / n_{i}=10^{-4}$, $v_{D}=2 v_{A}, p_{0} / m=300 v_{A}$. The top panel varies $n_{\mathrm{CR}} / n_{i}$, while the bottom panel varies $v_{D} / v_{A}$, as indicated in the legends. The growth rates for left (solid) and right (dashed) handed modes are shown separately. therein for motivation)

$$
f_{0}(p)=\frac{n_{\mathrm{CR}}}{\left(\pi \kappa p_{0}^{2}\right)^{3 / 2}} \frac{\Gamma(\kappa+1)}{\Gamma\left(\kappa-\frac{1}{2}\right)}\left[1+\frac{1}{\kappa}\left(\frac{p}{p_{0}}\right)^{2}\right]^{-(\kappa+1)},
$$

which has the property that $f(p)$ is a constant when $p \ll$ $p_{0}$, and $f(p) \propto p^{-2(\kappa+1)}$ as $p \gg p_{0}$.

The dispersion relation remains the same except with factors of $Q_{1}(k)$ and $Q_{2}(k)$ in Equation (5) replaced by

$$
\begin{gathered}
Q_{1}=\frac{\Gamma(\kappa+1)}{s_{0}^{3}(\pi \kappa)^{3 / 2} \Gamma\left(\kappa-\frac{1}{2}\right)} \int_{0}^{\infty} \frac{2 \pi s \cdot d s}{\left[1+\left(s / s_{0}\right)^{2} / \kappa\right]^{\kappa+1}} \log \left|\frac{1+s}{1-s}\right| \\
Q_{2}=\frac{\sqrt{\pi}}{\kappa^{3 / 2}} \frac{\Gamma(\kappa+1)}{\Gamma\left(\kappa-\frac{1}{2}\right)} \frac{1}{s_{0}\left[1+1 /\left(\kappa s_{0}^{2}\right)\right]^{\kappa}}
\end{gathered}
$$

where the dependence on $k$ is expressed in $s_{0} \equiv p_{0} / p_{\text {res }}=$ $k p_{0} / m \Omega_{c}$. The $Q_{1}$ integral can be evaluated numerically, and in the limit $n_{\mathrm{CR}} / n_{i} \ll 1$, the growth rate can be obtained analytically by substituting (40) into (7).

In Figure 3 (top panel), we show the linear growth rate in the same way as in Figure 2, but for $f_{0}$ being a $\kappa$ distribution. We see that in the long wavelength limit $\left(p_{\text {res }} \gg p_{0}\right.$ or $\left.s_{0} \ll 1\right)$, we have $Q_{2} \propto s_{0}^{2 \kappa-1}$. In the short wavelength limit, we have $Q_{2} \propto s_{0}^{-1}$. The results are consistent with a power law distribution function in the same limits, with $\alpha=2(\kappa+1)$. The growth rate varies smoothly in between the two limits, with peak growth rate only slightly reduced compared to the truncated power-law case. It is this analytical growth rate that we aim to test with further study.

We further show in the bottom panel of Figure 3 the analytical growth rate for different drift velocities at fixed $n_{\mathrm{CR}} / n_{i}=10^{-4}$. Increasing the drift velocity has a similar effect as increasing $n_{\mathrm{CR}} / n_{i}$, which increases the overall growth rate (roughly linearly). It also makes the righthanded mode stand out, which becomes more notable for $v_{D} \gtrsim 10 v_{A}$. In this paper, we restrict our explorations to $v_{D} \lesssim 8 v_{A}$.

\subsection{Simulation Box Size and Phase Randomization}

Even equipped with the $\delta f$ methods and a $\kappa$ distribution function, it is difficult to accurately recover the 
linear growth of the CRSI with a numerical simulation. Over a large suite of tests with wide range of numerical setups, we have found that sustained growth of the CRSI at the rate predicted by theory is only achieved when the simulation box size is extremely long. Otherwise, the desired growth rates are found only at very early stages, and they become erroneous shortly afterwards (we demonstrate this in Appendix C).

Further adjusting the numerical speed of light $\mathbb{C}$ reveals that in order to properly recover growth rates, the required simulation box length $L$ should be such that the time for the CR particles to traverse the simulation domain, $t_{\text {cross }}=L /(\mu \mathbb{C})$, exceeds the growth timescale for CRSI, $1 / \Gamma_{\max }$. Given that $\Gamma_{\max } \sim\left(n_{\mathrm{CR}} / n_{i}\right) \Omega_{c}$, and using Equation 16, this may be further translated to

$$
L>L_{\min } \equiv \frac{\mathbb{C} \mu}{\Gamma_{\max }} \sim \frac{n_{i}}{n_{\mathrm{CR}}} \frac{k_{\mathrm{res}}^{-1}}{\gamma} .
$$

This phenomenon is related to the validity of the random phase approximation for QLD discussed at the beginning of Section 3, which feeds instability growth. For CRSI, CR particles should constantly experience different wave packets throughout the growth process. With wave growth, we may regard the wave packets to be altered over the timescale of $\sim \Gamma_{\max }^{-1}$. With the fastest particles traveling at $\mathbb{C}$ along the background field, a simulation box longer than $L_{\text {min }}$ guarantees all particles experience "new" wave packets after traversing the entire box and reenter from the other side.

However, for reasonable simulation values of $n_{i} / n_{C R}$, the required $L_{\min }$ could easily reach a few hundreds or thousands times the resonant/most-unstable wavelengths (using $n_{i} / n_{C R} \sim 10^{9}$ as in the bulk ISM, the number could be billions). The condition of $L \gtrsim L_{\min }$ thus would impose a prohibitive requirement for simulating the CRSI, even in 1D.

We resolve this issue by realizing that the effect of long simulation box can be equivalently achieved by directly randomizing particle phases when a particle crosses the periodic boundary: both approaches allow particles to effectively see different wave packets at all times to conform to the random phase approximation. In our simulations, upon boundary crossing, we fix a particle's total velocity, as well as its parallel velocity along background magnetic field, whereas we randomize its gyro-phase. By this simple approach, we completely eliminate the requirement of $L>L_{\mathrm{min}}$. Also, under the $\delta f$ method, the phase randomization procedure does not introduce more errors to momentum/energy conservation.

\section{SIMULATION SETUP AND DIAGNOSTICS}

We set up the simulations in the frame of the "unperturbed" CRs, where their background distribution function is isotropic $f_{0}(\boldsymbol{p})=f_{0}(p)$. This is also the $f_{0}$ for the $\delta f$ method, with background CR current $\boldsymbol{J}_{\mathrm{CR}, 0}=0$. We set background density to $\rho_{0}=1$ and pressure $P_{0}=0.6$, with an ideal gas equation of state with adiabatic in$\operatorname{dex} \Gamma=5 / 3$ (so that sound speed $c_{s}=\sqrt{\Gamma P_{0} / \rho_{0}}=1$ ). While thermodynamics has no effect to the system, we adopt adiabatic rather than isothermal equation of state to better characterize energy conservation of the system (see Section 6.1.1). In 1D (along the $\hat{x}$ axis), the background magnetic field is set to $B_{0}=1$ along $\hat{x}$, so that the Alfvén speed $v_{A}=B_{0} / \sqrt{\rho_{0}}=1$.

For all simulations, we initialize the background gas to a velocity $\boldsymbol{v}_{0}=-v_{D} \hat{x}$. Since we are working in the frame where the CRs are initially isotropic, this means that the $\mathrm{CR}$ distribution streams to the right (along the direction of the mean magnetic field) with speed $v_{D}$ relative to the gas. On top of the background state, we initialize the system with a spectrum of Alfvén waves propagating along background $\boldsymbol{B}_{0}$. For each wavenumber $|k|$, there are four modes corresponding to forward and backward propagation, and left and right polarization. The wavenumber coverage ranges from $k=2 \pi / L_{x}$ to $k=2 \pi /(2 \Delta x)$ (except that the longest wave is initiated with 0 amplitude), where $L_{x}=N_{x} \Delta x$ is the simulation domain size, and $N_{x}$ and $\Delta x$ are the number of grid cells and cell size in $\hat{x}$. Correspondingly, a total of $4\left(N_{x} / 2-1\right)$ modes are initialized. We choose the wave amplitudes to be such that

$$
I(k)=A^{2} / k,
$$

so that wave energy is equally distributed in logarithmic $k$-space, and the total wave energy integrated over one dex in $k$ is a fraction $2 A^{2} \ln 10$ of the background field energy (cf. Equation 13; the factor of two accounts for velocity perturbations). Each mode is initialized with a random phase.

We choose code units such that individual CR particles have cyclotron frequency $\Omega_{c}=1$ in the background field $B_{0}$. This corresponds to the factor $q / m c=1$ in Equation (34). Thus, our code unit of length is $d_{i}=v_{A} / \Omega_{c}=1$. We note that while irrelevant to $\mathrm{MHD}, d_{i}$ is equivalent to the ion skin depth $c / \omega_{p i}$ for the background plasma. Our MHD-PIC framework typically applies to scales greater than $d_{i}$.

The CR particles are injected with $f_{0}(p)$ being a $\kappa$ distribution. We choose $\kappa=1.25$ throughout this work, corresponding to $f_{0}(p) \propto p^{-4.5}$, at large $p$ or $\alpha=0.5$ in Equation (8). In practice, we divide the momentum space into 8 bins ranging from $0.01 p_{0}$ to $100 p_{0}$, with half a dex per bin. We inject equal number $\left(N_{p}\right)$ of CR particles per cell per bin so as to guarantee sufficient number of particles in each bin (otherwise there would be too few particles towards lower and higher energies). Within each bin in each cell, particles are sampled according to $f_{0}(p)$ in that momentum range, and are symmetrically distributed in pitch angle and phase. More specifically, once a set of random momentum $p$, pitch angle $\theta$ and gyro-phase $\phi$ is generated, four particles are injected at cell center whose momenta are $(p \cos \theta, p \sin \theta \cos \phi, p \sin \theta \sin \phi)$ and its permutations with $\theta \rightarrow \pi-\theta$ and $\phi \rightarrow \pi+\phi$.

We note that particles with $p<0.01 p_{0}$ and $p>100 p_{0}$ are not included in the simulation whereas we still use a background distribution function $f_{0}$ that covers the entire momentum space. This will introduce some inconsistencies. However, since these momenta are sufficiently far from the peak of $f_{0}$, waves that they drive are typically beyond what can be accommodated in our simulation box (and moreover the simulation time is typically not long enough for these modes to grow). 


\subsection{Choice of Parameters}

The interstellar medium in our Galaxy spans a wide range of conditions in various phases. In a averaged sense, $n_{\mathrm{CR}} / n_{i}$ is on the order of $\sim 10^{-9}$, and $v_{A} / c \sim$ $10^{-5}-10^{-4}$, and the bulk of the energy is in GeV CR particles that are mildly relativistic. While the parameters that we adopt are by no means fully realistic (this would be unachievable), we aim to achieve as much scale separation as possible to mimic conditions in the largescale ISM.

We choose the artificial speed of light $\mathbb{C} / v_{A}=300$, and choose the characteristic momentum in the CR distribution to be $p_{0} / v_{A}=300=\mathbb{C} / v_{A}$. With this choice, Equation (33) yields $\gamma_{0}=\sqrt{2}$ so as to mimic the fact that the dominant CR population in the ISM is transrelativistic $\sim \mathrm{GeV}$ particles.

The fact that $\mathbb{C}=300$ is larger than $v_{A}=1$ by more than two orders of magnitude allows for sufficient separation, making the transformation between different reference frames well in the non-relativistic limit (e.g., a drift velocity as large as $\sim 10 v_{A}$ only amounts to about $3 \%$ of $\mathbb{C})$. With our choices, the wavelength for most unstable mode is given by $\lambda_{m} \approx 2 \pi / k_{0}=2 \pi p_{0} / \Omega_{c} \approx 1885$ in code units.

Based on Equation 7 for the growth rate of CRSI, the two main physical parameters are the CR number density ratio $n_{\mathrm{CR}} / n_{i}$, and the $\mathrm{CR}$ drift velocity $v_{D} / v_{A}$. As already seen in Figure 3 , given our choices of $\mathbb{C}$ and $p_{0}$, the density ratio $n_{\mathrm{CR}} / n_{i}$ has to be well below $10^{-3}$ to make left and right handed modes grow at about equal rates (as in standard CRSI). We thus choose $n_{\mathrm{CR}} / n_{i}=10^{-4}$ as fiducial, and vary it within $\left[10^{-5}, 10^{-3}\right]$. Note that further smaller values would make the waves grow too little within a reasonable computational time. We take $v_{D}=2 v_{A}$ for our fiducial model, and also consider higher drift velocities up to $8 v_{A} \cdot{ }^{4}$

The remainder are numerical parameters. Fiducially, we choose simulation box size $L / d_{i}=9.6 \times 10^{4}$, resolved by 9600 cells $\left(\Delta x / d_{i}=10\right)$. The fiducial box is about $\sim 50$ times the most unstable wavelength, which is more than sufficient for particles with $p \sim p_{0}$, but it better accommodates more energetic particles with $p \gtrsim 10 p_{0}$. Note that without implementing phase randomization, from Equation (41) the box size $L_{\min }$ would have to be $\sim 300$ times longer than our fiducial choice. We also conduct a simulation with shorter box size for comparison.

The level of noise in the simulation depends on the number of particles. Fiducially, we choose $N_{p}=256$ particles per momentum bin per cell (in total 2048 per cell), but we also consider a case with fewer particles. The initial wave amplitude is fiducially chosen to be $A=$

4 In terms of energy density, we have $E_{\mathrm{CR}} / E_{B} \sim 45$ under the fiducial parameters, which is much larger than the order-unity value that applies through most of the ISM. We note, however, that while the total energy density of CRs matters for large-scale ISM dynamics, it is not directly relevant for the CRSI because the free energy of the CRSI comes from CR anisotropy (i.e., streaming) rather than total $\mathrm{CR}$ kinetic energy. Nonetheless, with $E_{\mathrm{CR}} / E_{B} \sim$ $\left(n_{\mathrm{CR}} / n_{i}\right)\left(\mathbb{C} / v_{A}\right)^{2}$, our run with $n_{\mathrm{CR}} / n_{i}=10^{-5}$ better approaches the realistic limit. One can also reduce $\mathbb{C} / v_{A}$ to 100 or smaller to reduce this ratio, although this would limit the range of $v_{D}$ that can be more reliably covered by simulations.

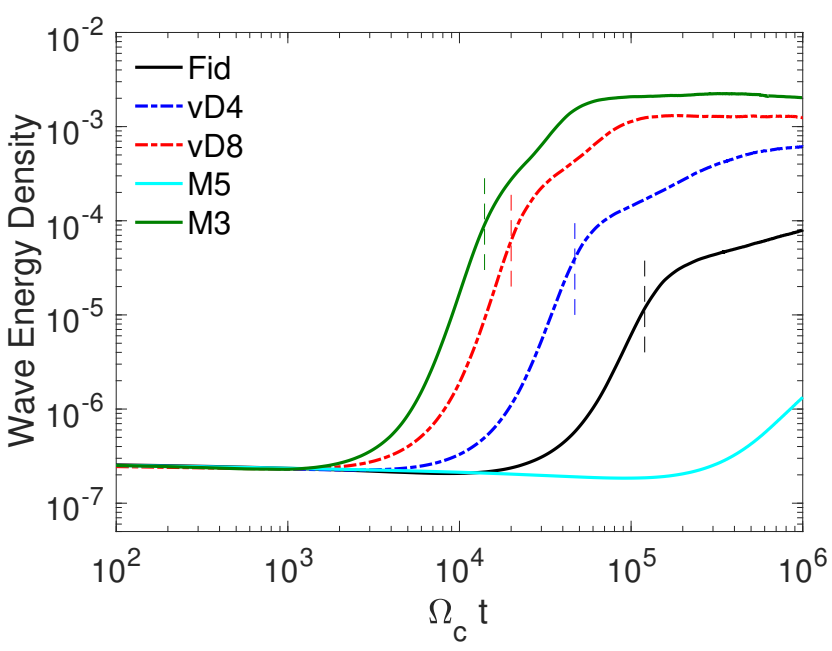

FIG. 4. - Time evolution of total wave energy in runs Fid (black), M3 (green), M5 (cyan), as well as runs vD4 (blue dotted), vD8 (red dotted). All in code units. Vertical dashed lines mark the end of the linear growth phase.

$10^{-4}$. Note that $A$ should not be too small, otherwise the initial evolution would be noise dominated. It should neither be too large, which would trigger artificial QLD of particles from the beginning. A more detailed discussion about noise in our simulations is given in Appendix B.

In Table 1, we list all simulation runs presented in this paper. We will primarily focus on fiducial run Fid and M3 (higher $n_{\mathrm{CR}} / n_{i}$ ). For other simulations, we generally vary just one parameter from run Fid at a time, and examine the role of both physical and numerical parameters. Note that in the simulations, almost all computational cost is spent on the CR parts given the large number of particles per cell. For our fiducial run, a typical timestep is $6 \times 10^{-2} \Omega_{c}^{-1}$, and the entire run to $t=10^{6} \Omega_{c}^{-1}$ costs about $4 \times 10^{4} \mathrm{CPU}$ hours on intel Xeon E5-2690 v4 CPUs.

\subsection{Diagnostics}

We first measure the linear growth rate of the CRSI. Over constant time intervals, we decompose the perpendicular magnetic field and velocity profiles into four Alfvén modes and obtain $I(k, t)$ for each mode (see Appendix A). A linear fit is then performed to $\ln [I(k, t)]$, with the slope being $2 \Gamma(k)$. This is done within early phases of evolution before QLD starts to modify the CR distribution function.

Another diagnostic is to directly measure the CR distribution function. Thanks to the $\delta f$ method, this again can be done with great precision. In the simulation frame, we simply compute $f(p, \mu)=f_{0}(p)+\delta f(p, \mu)$, where $f_{0}$ is analytic and $\delta f$ can be obtained in the same way binning particles onto a $p-\mu$ grid using the weighting scheme in Equation (35).

We can further combine the above information aiming to test QLD directly. This involves transforming $f(p, \mu)$ into the wave frame using Equation (19), and then solving Equation (14), with $I(k)$ feeding into the calculation. The results can be compared with the measured CR distribution function. 

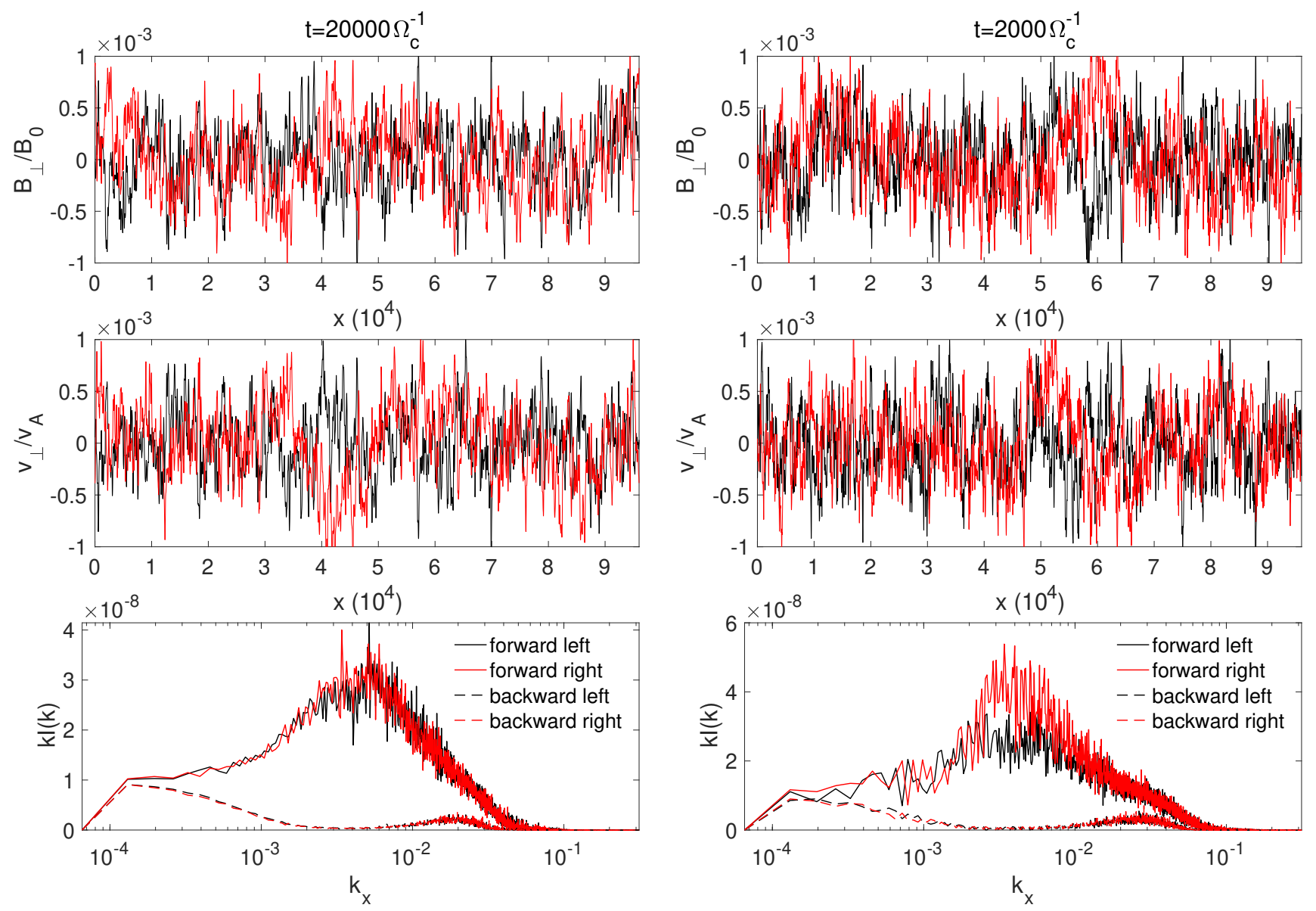

FIG. 5. - Snapshot at the linear growth phase in run Fid (left) and M3 (right). Top and middle panels show the profiles of the perpendicular components of magnetic field and velocity (black and red for the $y$ and $z$ components), normalized to $B_{0}$ and $v_{A}$. The bottom panel shows the wave spectra (dimensionless, in $k I(k)$ ) for forward (solid) and backward (dashed) propagating modes. Left/right handed branches are marked in black/red.

Finally, we have tracked a (small) subset of particles in certain runs over certain time intervals; we combine this information with the field data to investigate how particles overcome the $90^{\circ}$ barrier.

\section{SIMULATION RESULTS: RUN FID AND M3}

In Figure 4, we show the evolutionary history of total wave energy for the first six runs listed in Table 1. In this section, we first focus on the two main simulation runs: Fid and M3, and will also briefly discuss run M5. With wave amplitude $A=10^{-4}$, total wave energy integrated over the wave spectrum in $k$ - space, and summed over all four modes, is about $3.2 \times 10^{-7}$ (compared to 0.5 for background magnetic field energy density). Wave energy slowly decreases at first, which is due to numerical damping of high- $k$ modes at grid scale, as well as damping of backward propagating modes. Shortly afterwards, exponential growth of the CRSI becomes clear, leading to rapid increase of wave energy. This continues to time $t \sim 10^{5}$ for run Fid and $t \sim 10^{4}$ for run M3, after which wave growth slows down as QLD starts to substantially modify the particle distribution function. Subsequent growth is slower, and is characterized by a combination of saturation of the fastest growing modes excited by low-energy particles, and the linear growth of longer wavelength modes resonant with (less abundant) higher-energy particles. Eventually, for run M3, the wave energy plateaus, marking system saturation. The wave energy density in the saturated state for run M3 is comparable to the estimates in Equation (25). However, for run Fid, in which the saturated energy density should simply be one order of magnitude smaller, this is not yet reached.

Clearly, the wave energy evolution is much better discussed on the $k$-by- $k$ basis, and should be accompanied by the evolution of particle distribution function. These will be addressed in the following subsections, for individual evolutionary phases.

\subsection{Linear Wave Growth}

In Figure 5, we show the Alfvén wave spectrum (in wave intensity $k I(k)$ for the four modes (forward/backward propagation, left/right polarization) at some early times during the linear phases for the two runs. Initial wave intensity of all modes has $k I(k)=10^{-8}$ being constant, with $\delta v / v_{A} \sim \delta B / B_{0}$ at the level of a few times $10^{-4}$. Modes with $k \gtrsim$ a few times $10^{-2}$ are numerically damped. With very low numerical noise, 


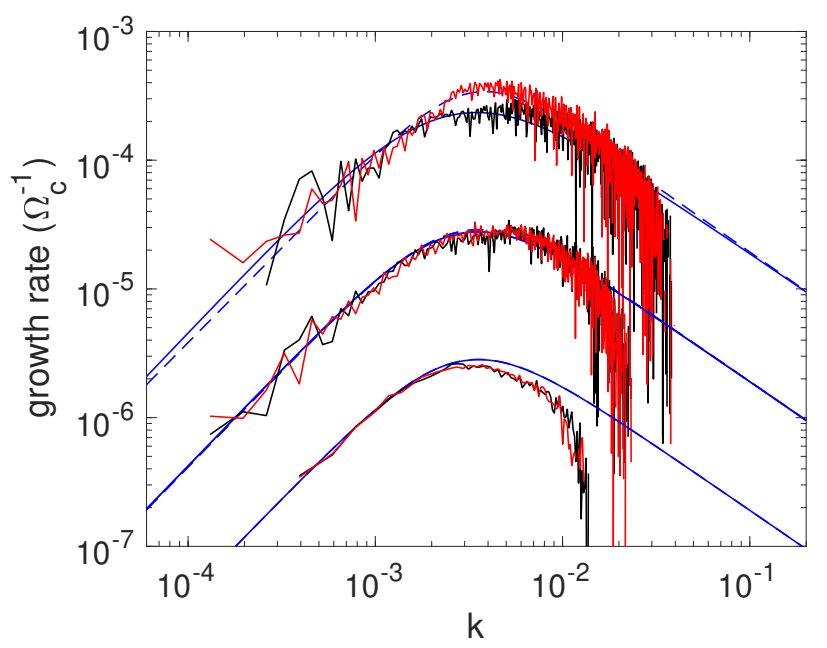

FIG. 6. - The linear growth rate of the CRSI measured in run M3 (top), Fid (middle) and M5 (bottom), where black/red curves mark the left/right handed polarization. Blue solid/dashed lines mark the analytical growth rate expected from a $\kappa$-distribution for left/right handed modes.

linear growth of all other forward-propagating modes is observed from the beginning, whereas backward propagating modes always damp. The fastest growing mode, resonant with particles near $p=p_{0}$, is found to be near $k=k_{m} \approx 3.3 \times 10^{-3}=\Omega_{c} / p_{0}$ as expected.

More quantitatively, we measure the linear growth rate of forward-propagating modes on the $k$-by- $k$ bases. This is done within $t=3 \times 10^{4} \Omega_{c}^{-1}$ for run Fid and $t=10^{4} \Omega_{c}^{-1}$ for run M3. We also consider run M5, and measure its growth rate within $t=5 \times 10^{5} \Omega_{c}^{-1}$. The results are shown in Figure 6, and are compared with analytical growth rates.

Overall, even though the modes are very densely populated, the numerical growth rate matches analytical results remarkably well. For runs Fid and M5, both left and right handed modes grow at about the same rate, whereas for run M3, right-handed mode grows slightly faster, all being consistent with theoretical expectations. We stress that phase randomization is essential in achieving these results, as is further discussed in Appendix C.

The growth of the CRSI is better captured towards low $k$, whereas for high $k$, instability growth competes with wave damping by numerical dissipation. As a result, the measured growth rates cut off at some $k \sim k_{\text {cut }}$, which decreases from run M3 to run M5 as the instability growth rate decreases by about two orders of magnitude. In fact, because of the small CRSI growth rate, $k_{\text {cut }}$ corresponds to about 20,30, and 45 grid cells for runs M3, Fid, and M5. This again indicates much higher numerical resolution is needed to study the CRSI compared with typical pure MHD instabilities.

Note that for run M3, although the difference in growth rate between left and right handed modes is small, the resulting difference in wave amplitudes can be significant after a few e-folding times. This is already evident in the right bottom panel of Figure 5: within one e-folding time, the intensity of the right handed mode is already about twice that of the left handed mode. This will affect subsequent QLD of particles to be discussed later.

As a side note, we have also conducted simulations with drift speed $v_{D}$ between 0 and $v_{A}$. We find that all wave modes are damped, and damping is the fastest for at $k \sim k_{m}$. In the case of $v_{D}=0$, forward/backward propagating modes damp at the same rate, whereas for $0<v_{D}<v_{A}$, damping of forward propagating modes are slower than that for backward propagating modes. They all agree well with the dispersion relations.

\subsection{Quasi-Linear Evolution for run Fid}

Past the linear stage, QLD starts to modify the particle distribution functions, and drives the particle distribution towards isotropy in the wave frame. In Figure 7, we show the time evolution of the particle distribution function $\left(\delta f / f_{0}\right)$ via four successive snapshots of the our fiducial run. For each snapshot, the color scale represents occupation at a given $p$ and $\mu$. We have also marked with dashed lines the loci in phase space resonating with the same wavelength

$$
p \mu=\Omega_{c} / k=\text { const . }
$$

From Figure 7, it is clear that over time a deficit of particles develops near $\mu \sim 1$ while an excess develops near $\mu \sim-1$. This change in $\delta f$ reflects the tendency of QLD to scatter CRs towards a new distribution that is isotropic in a frame that is moving to the left with respect to the initial CR distribution. Figure 7 also shows that an excess and deficit of particles develops respectively to the right and left of $\mu=0$; we discuss this further below.

In Figure 8 we show the wave spectrum for the same snapshots as Figure 7. In these plots, we mark with dashed vertical lines the $k$ values for the corresponding dashed lines in Figure 7. Over time, the spectrum of forward-propagating waves grows at all but the lowest and highest $k$, with comparable amplitudes for left- and right- polarizations.

\subsubsection{Overall Evolution}

At $t \sim 3$ to $10 \times 10^{4} \Omega_{c}^{-1}$, QLD has started to make appreciable changes to the particle distribution function. The initial QLD is the fastest around particles in resonance with the fastest growing mode at $k \sim k_{m}=$ $3.3 \times 10^{-3}$, as can be clearly identified in the second panel of Figure $7 .^{5}$ As discussed in Section 2.1, the way QLD acts to isotropize the $\mathrm{CR}$ distribution is by scattering forward-traveling CRs to from higher to lower (positive) parallel velocities, and by scattering backward-traveling CRs from lower to higher (negative) parallel velocities. This is exactly what we see in Figure 7 , with excess and deficits on the left and right sides of the thick black dashed line.

With some CRs resonant with these waves giving up their free energy through QLD, the growth of the most unstable mode slows down. More slowly growing modes at longer wavelengths have not yet grown to sufficient amplitudes to yield appreciable QLD to particles in resonance with them at this time, thus still grow at the

\footnotetext{
${ }^{5}$ The initial wave spectrum also leads to some QLD that slightly affects the appearance of the distribution function (owing to contributions from high- $k$ modes) at early times $t \lesssim 5 \times 10^{4} \Omega_{c}^{-1}$.
} 

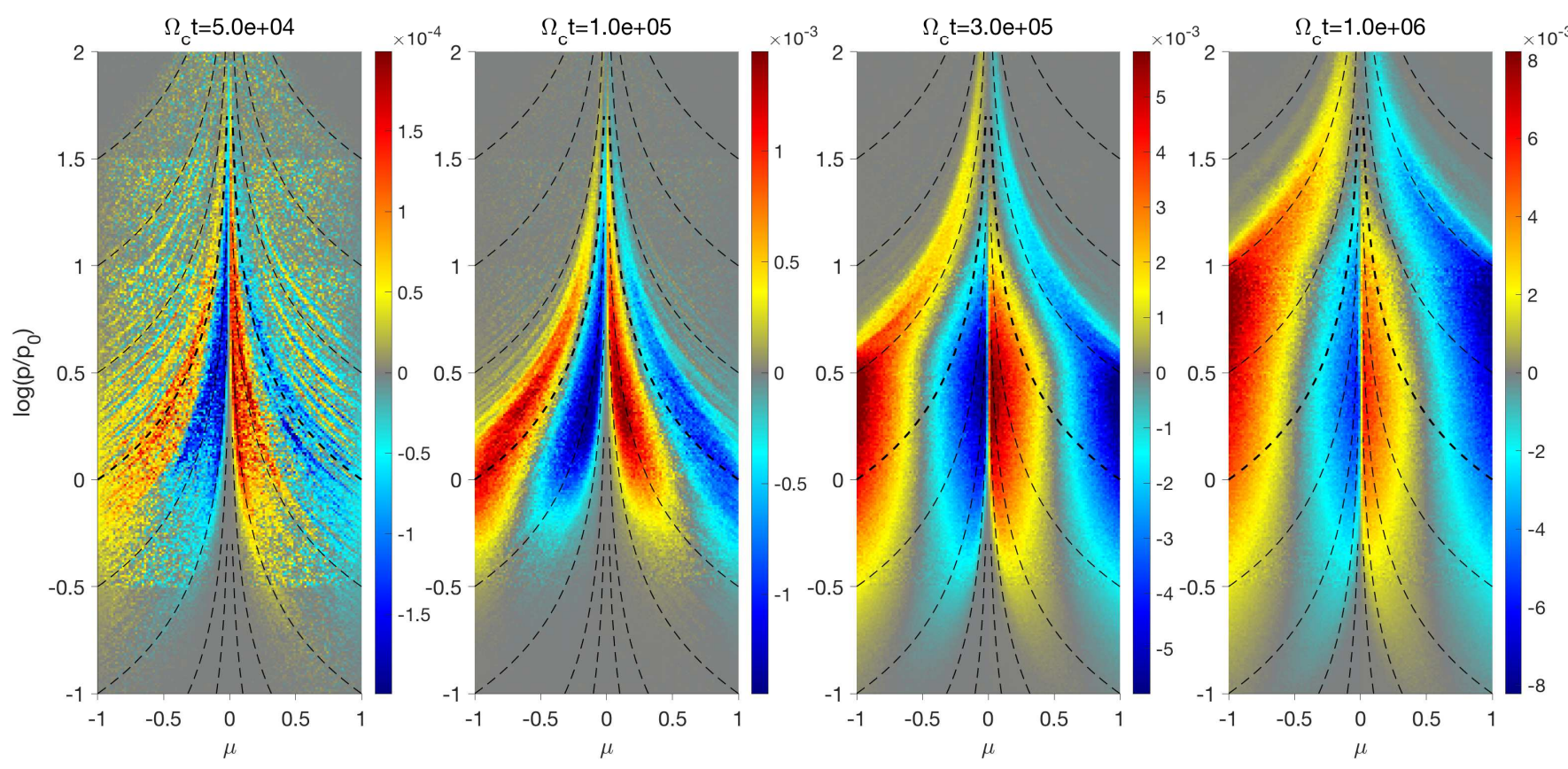

FIG. 7. - The 2D distribution function $\delta f / f_{0}$ in the lab (simulation) frame at four snapshots in run Fid. The dashed lines are contours in momentum space that are resonant with the same wave $\left(p \mu=\right.$ const), with the thick line marking $p \mu=p_{0}$. Note that color ranges are different among different panels.

same rate. As a result, the peak of the wave spectrum shifts towards longer wavelength, as seen from the time sequence in Figure 8.

Such wave growth then leads to further QLD for more energetic particles, as can be seen in the later two panels of Figure 7. By the end of our simulation at $t=10^{6} \Omega_{c}^{-1}$, all particles with $p \lesssim 10 p_{0}$ have undergone substantial QLD, whereas for particles with $p>10 p_{0}$, only the ones with smaller pitch angle have undergone some QLD. While the trend should continue over time, we are also limited by the simulation box size, with corresponds to 50 times the most unstable wavelength. Modes available for scattering particles with $p>10 p_{0}$ are fairly limited and discretized, precluding following QLD accurately (the discreteness can already be seen in the last panel of Figure 7). We thus do not run this simulation for longer.

Across $\mu=0$, there is substantial excess (deficit) of particles with $\mu>0(\mu<0)$ for $p_{0} \lesssim p \lesssim 10 p_{0}$, which clearly illustrates the $90^{\circ}$ pitch angle problem. Evidently, redistribution of particle pitch angle occurs only on either side of $\mu=0$ but particles do not scatter across $\mu=0$. In part, the failure to scatter across $\mu=0$ owes to lack of wave power at large $k_{x}$; particles with small $|\mu|$ would only be able to resonate and scatter off of shortwavelength waves. In our simulation, $k_{x}>0.1$ waves have very low amplitude due to damping (see Figure 8; waves at $k_{x}>\pi N_{x} / L_{x}$ are not resolved at all). In addition, for run Fid the amplitude $\delta B / B_{0}$ is insufficient to trigger reflection near $m=0$ for the bulk CR particles. We further discuss the circumstances that lead to "pre-mature" saturation in the next subsection.

Particles with $p \lesssim p_{0}$ have also undergone QLD, which is caused by shorter-wavelength modes with $k>k_{m}$. These modes initially grow slower than the fastest grow- ing mode at $k=k_{m}$, but they catch up towards later time, and eventually saturate at similar amplitudes, as can be seen from Figure 8. Correspondingly, the evolution in the distribution function also propagates towards lower $p$ side, as seen in Figures 7. However, this is eventually limited by numerical dissipation. Given our resolution, we already see from Figure (6) that modes with $k \gtrsim 6 k_{m}$ grow much slower than theoretical expectation due to numerical dissipation. Considering scattering across the entire pitch angle range, and given that even particles with $p \sim p_{0}$ are subject to pre-mature saturation, we do not discuss particles with $p<p_{0}$ in general.

We have also examined run M5. The overall evolution is largely similar to run Fid: the evolutionary stage at time $t$ in run M5 is similar to that at time $t / 10$ in run Fid, which is also visible from Figure 4. This is reasonable, given the exactly factor of 10 difference in wave growth rates. QLD starts to modify the $\mathrm{CR}$ distribution function after $t \sim 3 \times 10^{5} \Omega_{c}^{-1}$, and by the end of the simulation at $t=1.5 \times 10^{6} \Omega_{c}^{-1}, \mathrm{QLD}$ is already close to being completed for particles with $p \sim 1-3 p_{0}$, except that they are stuck at $\mu=0$. Given even smaller wave amplitudes in run M5 than in run Fid, we do not expect further evolution would help with this $90^{\circ}$ problem.

\subsubsection{Quantitative Analysis}

To see how well our simulation results compare with the quasilinear theory, we transform the distribution function to the wave frame using Equation (19). For each particle bin $\left(p_{w}, \mu_{w}\right)$ at a given snapshot, we can find its resonant wave number $k_{\text {res }}$ and its corresponding $I\left(k_{\text {res }}\right)$, and compute the scattering frequency $\nu$ from Equation (15). Then, we solve the QLD Equation (14) over time numerically, with $I(k)$ being updated over a time interval of $\Delta t=500 \Omega_{c}^{-1}$ (which is the frequency of 

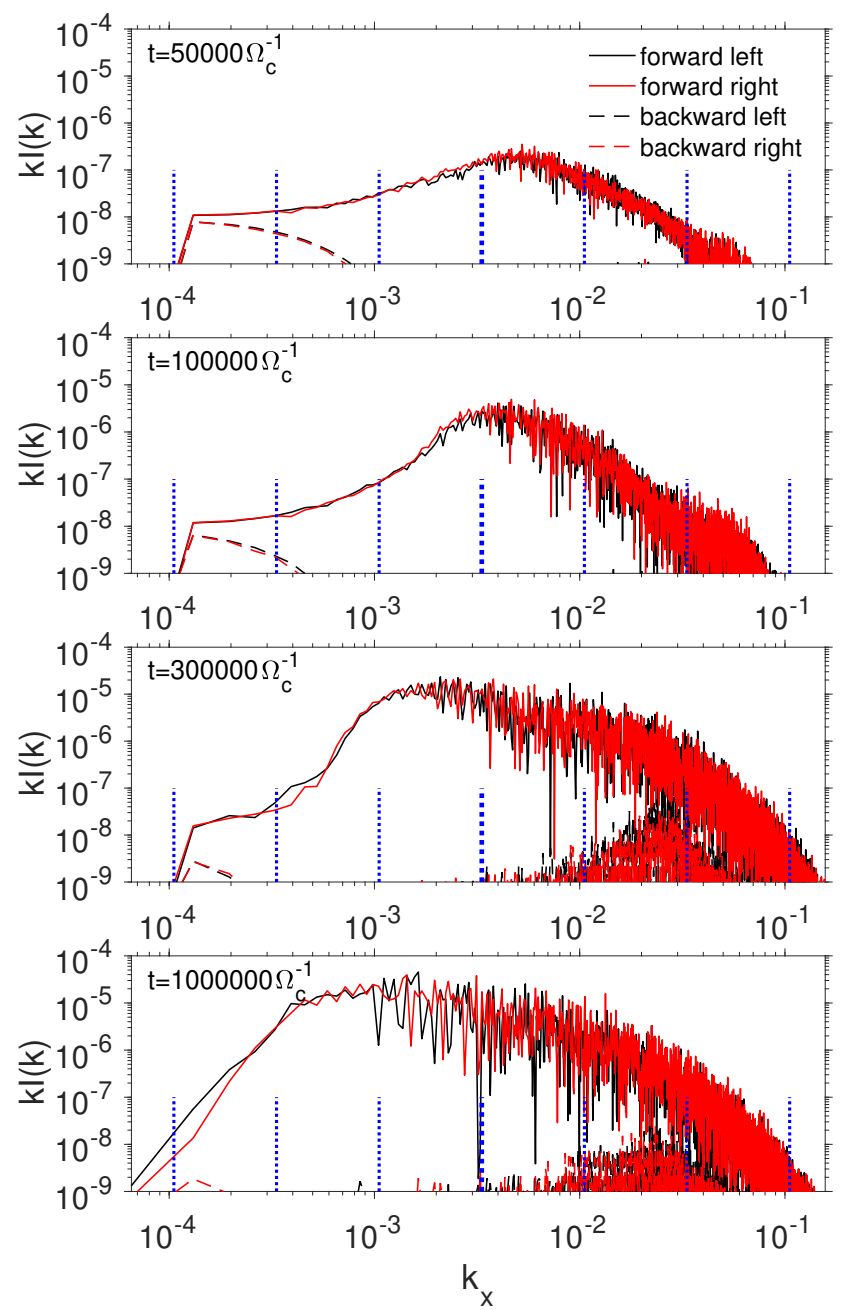

FIG. 8.- Wave spectrum at different times during QLD in run Fid. The vertical dotted lines mark the wave numbers that particles located at the black dashed lines in Fig. 7 can resonate with.

simulation data output).

In Figure 9, we show four snapshots in this process. Note that for better comparison, we have chosen snapshots 1,3 and 4 to correspond to snapshots $2,3,4$ in Figures 7 and 8 . The scattering frequency gradually increases to the level of $\nu_{\max } \sim 10^{-4} \Omega_{c}$ as the waves grow, and its appearance in $p-\mu$ space again shows the characteristic features corresponding to $p \mu=$ const from the resonance condition (modulo that $\nu$ decreases towards larger $p$ as particles become relativistic). With short wavelength modes being damped by numerical dissipation, the scattering frequency for small values of $|p \mu|$ has a cutoff, leaving a gap around $\mu=0$ that is broader towards smaller $p$. This causes the $90^{\circ}$ barrier for QLD, where no particles can cross $\mu=0$, as is the case when solving Equation (14).

In the bottom panel, we show the particle distribution function in the wave frame. Initially, $f_{w}-f_{0}$ is simply given by Equation (19) taking $\delta f=0$, which is linear in $\mu_{w}$. Note that for particles with different momenta, both $\partial \ln f_{0} / \partial \ln p$ and $v_{w}$ can be different, yielding different slopes. With the $\kappa$ distribution, the slope is smaller for smaller $p$, and approach a constant $2(\kappa+1) \Delta v / c$ for rel- ativistic particles (thus the red and green dotted lines overlap). Subsequent evolution has already been discussed in Section 5.2.1 in the simulation frame, which is straightforward to be translated to the wave frame. Several important results and features are worth noticing.

First, at early time $\left(t \lesssim 2 \times 10^{5} \Omega_{c}^{-1}\right)$, evolution of the numerical distributions follow the QLD prediction remarkably well. This gives us confidence that our simulations properly capture QLD, thanks to the phase randomization technique that we employed.

Second, QLD drives the local slope of $f_{w}-f_{0}$ towards zero on either side of $\mu=0$ [see Equations (20), (21)]; piecewise flattening is achieved at later stages of evolution, reached by lower energy particles first. This marks the saturation of the CRSI in the local momentum space.

Third, particles undergoing QLD tend to get stuck at $\mu=0$. Eventually, the CRSI saturates because $f_{w}=$ const on both sides of $\mu=0$ for each $p$, although there is a discontinuous jump at $\mu=0$. This is the hallmark of pre-mature saturation of QLD.

Fourth, solving the QLD equation allows us to well reproduce the saturated state for lower-energy particles $\left(p \lesssim 3 p_{0}\right)$, but this is not true for higher energy particles $\left(p \gtrsim 10 p_{0}\right)$. Given that the only missing ingredient in the QLD equation (14) is the process that governs the crossing of $90^{\circ}$ pitch angle, this indicates that the $\mu=0$ crossing does happen (slowly) for higher-energy particles in our simulations.

Given the above results, we now check the requirements for QLD to scatter particles to the critical pitch angle such that the condition for mirror reflection (27) can be satisfied. Seen from Figure 4, we have the rms $\delta B / B_{0} \sim 9 \times 10^{-3}$, giving $\mu_{\text {mir }} \sim 6 \times 10^{-3}$. Within our simulation time of $10^{6} \Omega_{c}^{-1}$, from Equation (14) we need $k I(k) \gtrsim 10^{-6}$ for effective QLD. Reading from Figure 8, this gives a maximum wave number $k_{\text {cut }} \sim 8 k_{m}$. Based on resonance conditions, the minimum momentum for which QLD can scatter the particle to reach $\mu=\mu_{\text {mir }}$ is then

$$
p_{\text {min }} \sim \frac{k_{m}}{\mu_{\text {mir }} k_{\text {cut }}} p_{0} \sim 20 p_{0} .
$$

In other words, waves in our simulation only have sufficient power to scatter particles with $p \gtrsim p_{\min }$ to reach pitch angle $\mu_{\mathrm{mir}}$, potentially allowing the mirror effect to yield reflection (and deviate from pure QLD result). However, from the bottom right panel of Figure 9 representing the last snapshot of the simulation, we see that even for $p$ as small as $\sim 3 p_{0}$, deviation from QLD can be identified, though just by a very small amount. Reading off the figure, deviation from QLD appear to begin for $p_{\min }$ between $3 p_{0}$ and $10 p_{0}$, which is in tension with the expectation from mirror reflection (Equation 44). Further discussion of this issue is deferred to Section 5.4, where a more detailed study based on run M3 is presented.

\subsubsection{Evolution of CR Drift Velocity}

While it is generally convenient to use the reduction of CR bulk drift velocity to describe how much CR particles have undergone scattering through the CRSI, this drift 

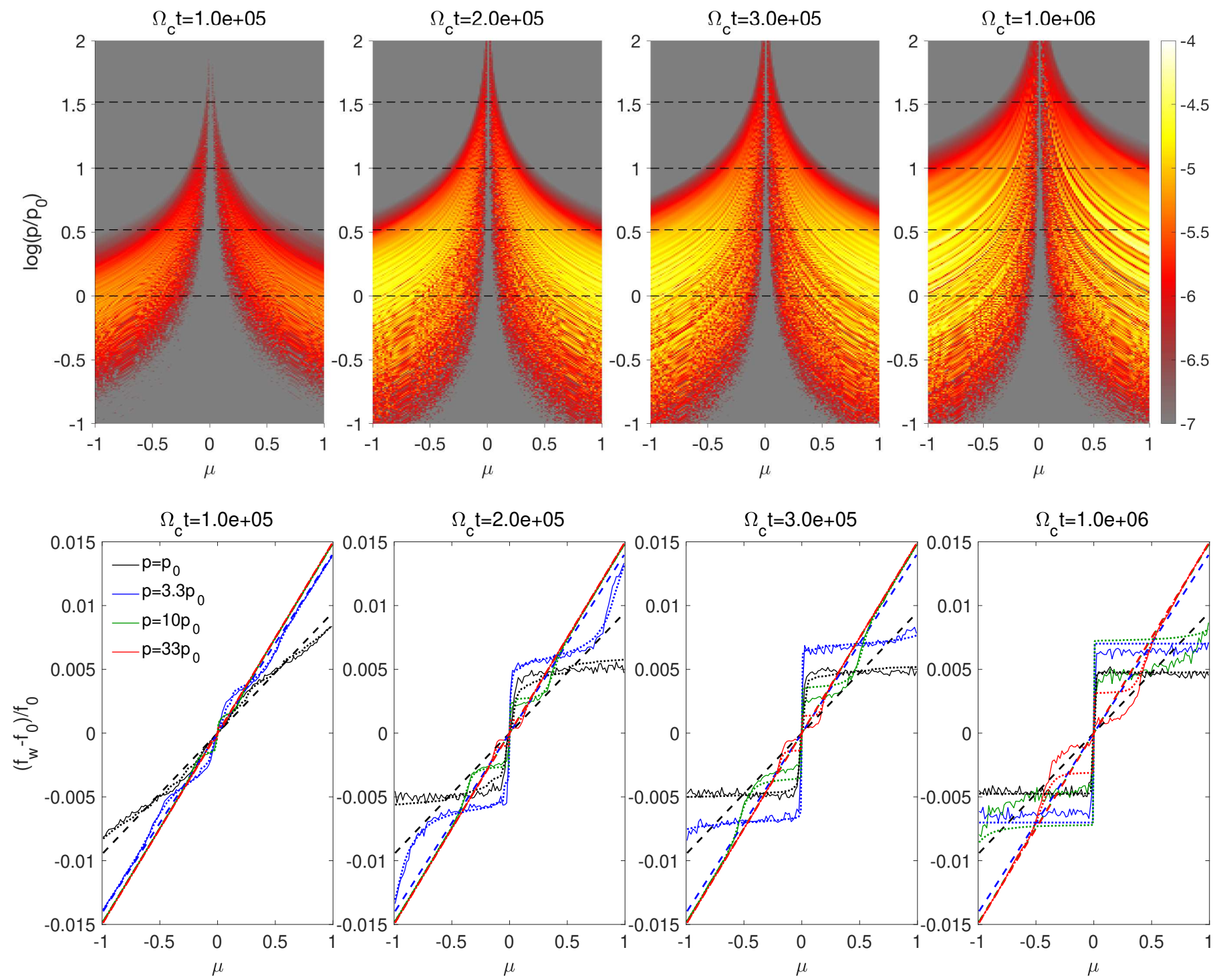

FIG. 9.- Top: four sequential snapshots of the scattering frequency $\nu$ of QLD (see Equation 15) in momentum space in run Fid. Shown in color is $\log _{10}\left(\nu / \Omega_{c}\right)$. Bottom: particle distribution function in the wave frame $f_{w}$ at the same four snapshots as in the top panel. Shown are $\left(f_{w}-f_{0}\right) / f_{0}$ for four representative momenta as a function of pitch angle cosine $\mu$, indicated by different colors (see legend). These momenta are also marked as black dashed lines in the top panel. Solid lines are measured from the simulations, thick dashed lines mark the initial distribution, while the dotted lines are reconstructed distribution functions by solving the QLD Equation (14) in time.

velocity is clearly energy-dependent. We can evaluate the energy-dependent drift velocity $v_{d}(p)$ (to distinguish from the initial CR bulk drift velocity $v_{D}$ ), in the simulation frame, as

$$
v_{d}(p)=\frac{\int f(p, \mu) v(p) \mu d \mu}{\int f(p, \mu) d \mu},
$$

where $v(p)$ is the velocity that corresponds to momentum $p$, and $f=f_{0}+\delta f$. Values of $v_{d}(p)$ can be simply obtained integrating over a horizontal line in Figure 7. We can further integrate the distribution function over $p$, to obtain the full drift velocity $v_{d, \text { full }}$.

In the left panel of Figure 10, we show the results for four different representative particle momenta from $p=p_{0}$ to $p=33 p_{0}$. Based on the previous discussion, the results are straightforward to interpret. The initial drift velocity in the simulation frame is by definition 0. Low-energy CRs, which are responsible for driving faster wave growth, undergo more rapid initial QLD and a corresponding reduction their drift speed; higherenergy CRs take much longer time to catch up. However, the CRSI and drift reduction of low-energy CRs saturates pre-maturely, leading to only modest reduction of $v_{d}$, whereas higher-energy CRs $\left(p \gtrsim 10 p_{0}\right)$ can overcome the $\mu=0$ barrier and are on the way to achieve complete isotropy. The overall average drift $v_{d \text {,full }}$ decreases over time. Contribution to the overall reduction is mostly from lower-energy particles with momenta between $\sim p_{0}$ and $\sim 3 p_{0}$ at early times, and from higher-energy particles with $p \sim 10 p_{0}$ at late times.

The drift velocity is frame-dependent. Similar to the above procedure, we can measure the drift velocity in the 

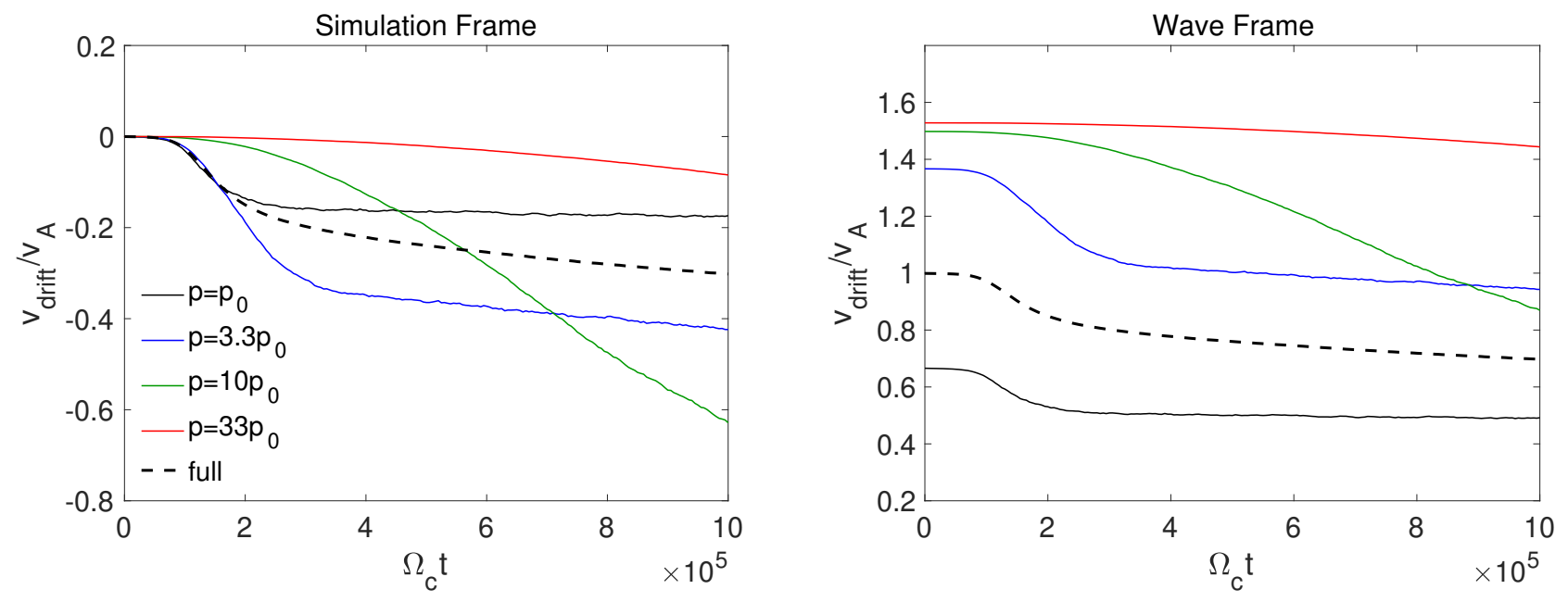

FIG. 10. - Time evolution of mean particle drift velocity in different momentum bins, together with the full drift velocity (see legend), for run Fid. We show velocities measured in the simulation frame (left) and wave frame (right). Thick dashed line represent the mean drift velocity averaged over the entire particle population.

wave frame $v_{d, w}(p)$, with the results are shown in the right panel of Figure 10. While the velocity difference between the two frames is just $v_{A}$, initial particle drift speeds within individual momentum bins do not equal $v_{A}$. This is because after a frame transformation, particles with a given momentum $p$ come from a range of momenta near $p$ from the original frame, making the results depend on $d \ln f_{0} / d \ln p$ (see Equation (19)). Only the

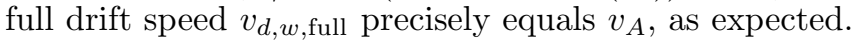
On the other hand, in a fully saturated state, we would expect $v_{d, w}(p)=0$ for all $p$. However, we see clearly from the Figure that low-energy particles are stuck at nonzero $v_{d, w}(p)$ whereas higher-energy particles require a longer time to achieve saturation.

\subsection{Quasi-Linear Evolution for run M3}

With $n_{\mathrm{CR}} / n_{i}$ a factor of 10 larger, run M3 evolves much more rapidly than run Fid. We choose four representative snapshots at $\Omega_{c} t=10^{4}, 3 \times 10^{4}, 10^{5}$ and $9 \times 10^{5}$ and analyze the results.

\subsubsection{Overall Evolution}

In Figure 11, we show the particle distribution function at these snapshots, while Figure 12 shows the corresponding wave intensity spectra. As discussed earlier, right-handed modes grow faster near $k \sim k_{m}$. This continues to $t \gtrsim 10^{4} \Omega_{c}^{-1}$. Since these modes are resonant with backward-traveling CRs, we see that at early time, QLD proceeds much more rapidly for $\mu<0$ particles resonant with these waves, leading to an initial asymmetry in the particle distribution function.

With asymmetric wave growth and QLD, backward traveling CRs have used up more free-energy, thus slowing down the growth of right-handed waves. The lefthanded modes, on the other hand, continue to grow normally. This allows left-handed modes to eventually catch up with the right-handed modes, and we see that by the time $t=3 \times 10^{4} \Omega_{c}^{-1}$, left and right handed modes at all wavelengths have similar amplitudes. At the same time, the distribution functions for forward and backward traveling CRs also tends to become more symmetric about $\mu=0$. However, the $\mu=0$ barrier remains at this stage for low-energy particles.

In between $\Omega_{c} t=3 \times 10^{4}$ and $10^{5}$, we see that crossing of the $\mu=0$ barrier occurs, paving the way for full saturation. The release of more free energy has led to some further wave growth. This process continues until the end of our simulation, with QLD continuing to extend to higher and higher energies.

\subsubsection{More Detailed Analysis Towards Saturation}

As before, for run M3 we compute the CR drift velocity in four different momentum bins for both the simulation frame and the wave frame, and show the results in Figure 13. We see that in the simulation frame, particles of all four momentum bins eventually flatten at different levels of $v_{d}(p)$. In the wave frame, they all reach $v_{d}(p)$ equal to zero $^{6}$, indicating that they are fully isotropized and have achieved complete saturation.

This saturation is more evident from Figure 14, showing a time sequence of the full distribution function in the wave frame, with the same snapshots as in previous Figures 11 and 12 . We can clearly identify the isotropization of particles in the later two snapshots, extending from the range of $p_{0} \lesssim p \lesssim 10 p_{0}$ at $t=10^{5} \Omega_{c}^{-1}$ to covering most of the particle population at the end of the simulation. Because of this, and with most of the particle population having momentum close to $p_{0}$, we see that the full drift speed $v_{d, \text { full }}$ averaged over all particle population shown is reduced almost exactly by $v_{A}$ (Figure 13 ).

For run M3, we have also computed the scattering frequency according to Equation (15) and solve the QLD equation (14) based on output data, and show the re-

6 The reason that the saturated drift velocity in the wave frame is not exactly zero in Figure 13 is purely numerical, depending on the resolution employed in binning particle momentum during simulation output (due to the sensitive dependence of $f_{0}$ on $p$ ). Finer binning makes the results closer to zero but with more noise, and vice versa. 

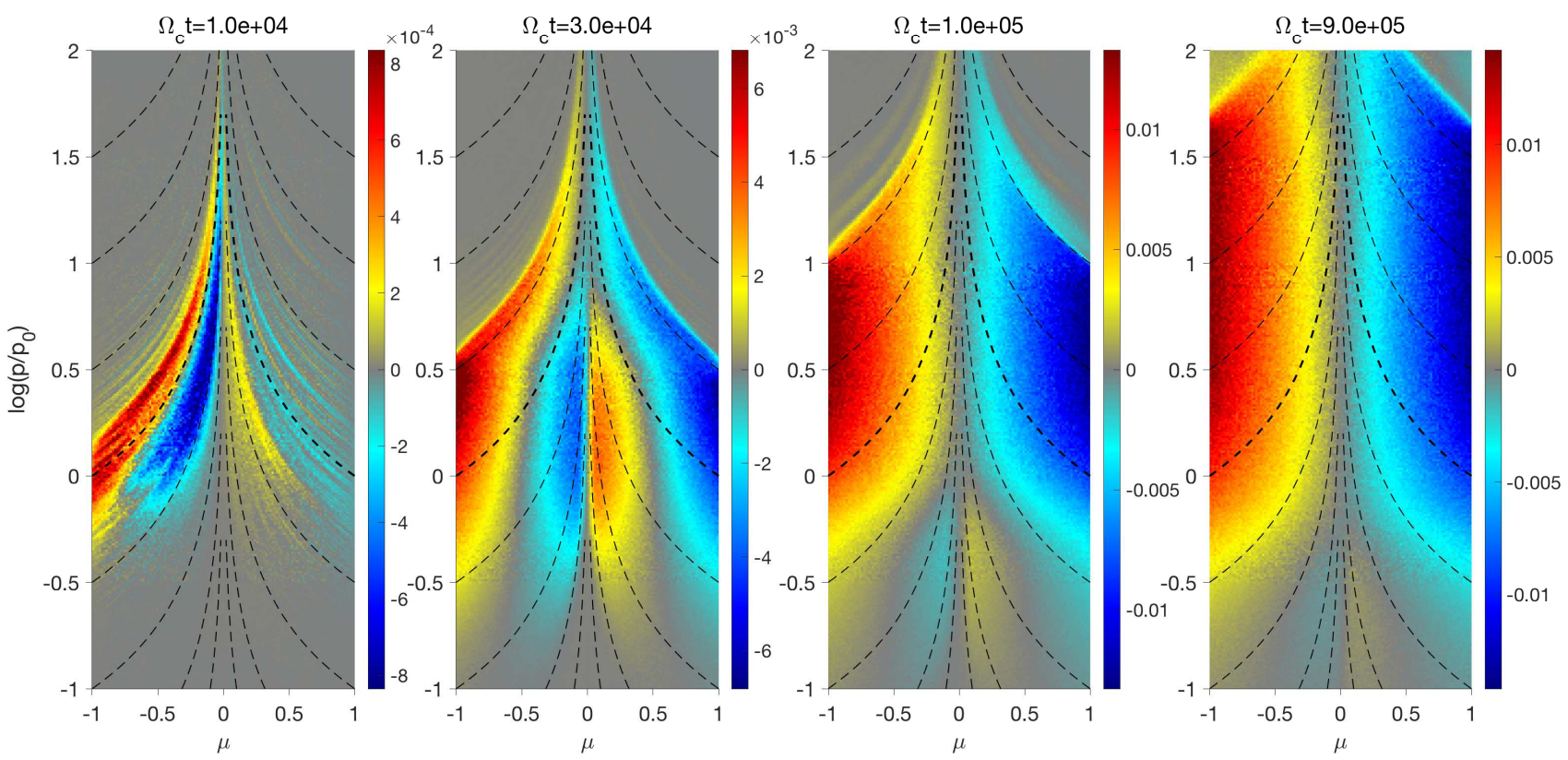

FIG. 11. - Same as Figure 7, but for run M3, showing the 2D distribution function of $\delta f / f_{0}$ in the simulation frame at four snapshots.
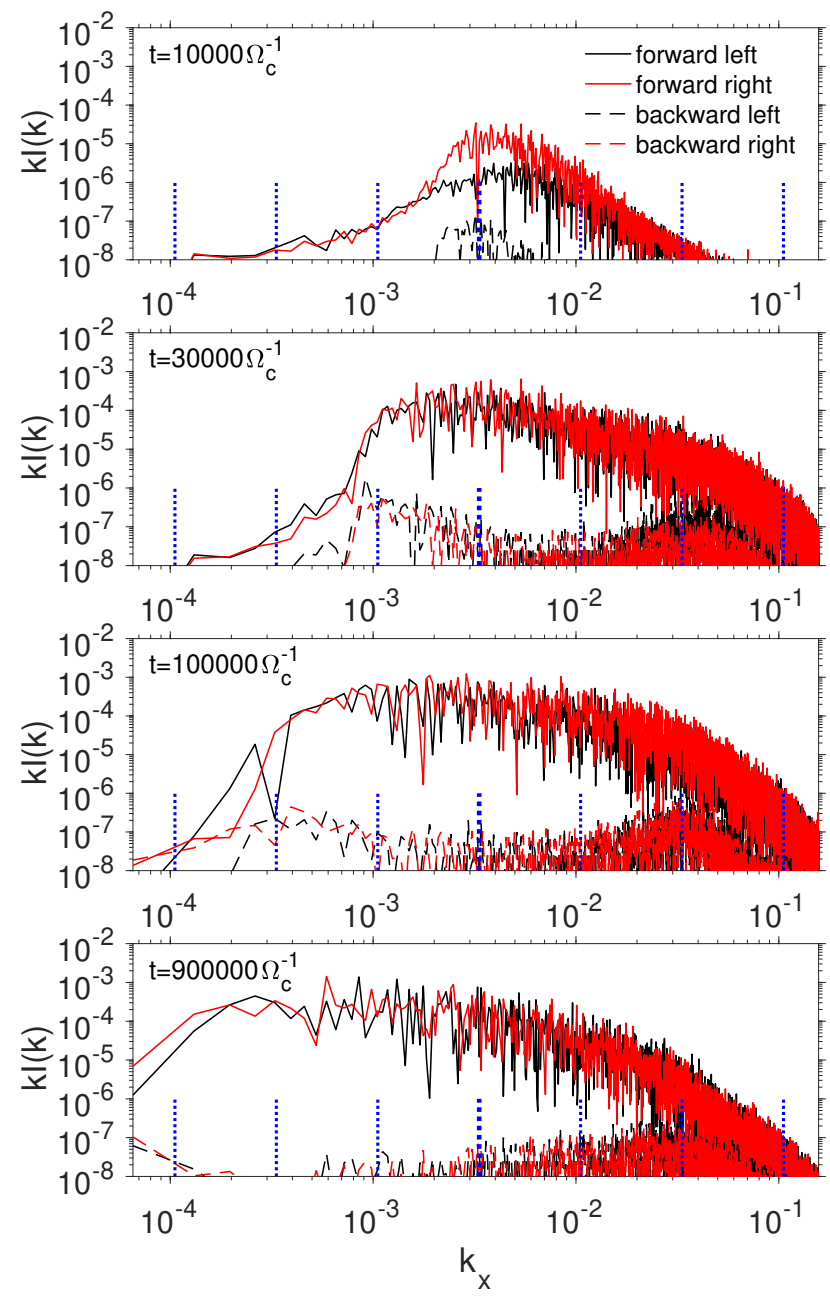

Fig. 12.- Same as Figure 8, but for run M3, showing the wave spectrum at the same four snapshots as in Figure 11. sults at four different snapshots in Figure 15. The asymmetry in scattering of forward/backward traveling CRs at early times $\left(t \sim 10^{4} \Omega_{c}^{-1}\right)$ can be clearly identified in the leftmost upper panel. At later times, we can see by comparing with Figure 11 that the extent to which QLD has proceeded in momentum space is strongly related to the region where scattering frequency is high.

The bottom panels of Figure 15 give more quantitative information about how quasi-linear evolution has progressed over time. For lower-energy particles (with $\left.p \sim p_{0}-3 p_{0}\right)$, evolution proceeds rapidly and can initially be well reproduced by solving the QLD equation. During subsequent evolution, particle distribution functions in fixed momentum bins are largely flat (due to rapid QLD) on both sides with a jump at $\mu=0$. The jump gradually decreases, as particles get scattered across $\mu=0$, which is the rate-limiting process for particle isotropization. For higher-energy particles $\left(p \gtrsim 10 p_{0}\right)$, on the other hand, crossing of $\mu=0$ is more rapid (leading to substantial deviation from directly solving the QLD equation). The rate-limiting process for isotropization then becomes QLD itself, especially at large $\mu$.

A key result from our run M3 is efficient particle crossing of the $\mu=0$ barrier. We again first examine whether this is consistent with mirror reflection. From Figure 4 , we see that by the time of $t=6 \times 10^{4} \Omega_{c}^{-1}$, the magnetic energy density approaches saturated value $\left(\delta B^{2} / B_{0}^{2} \sim 2 \times 10^{-3}\right)$, corresponding to a $\delta B / B_{0} \sim 0.045$. This gives the mirror reflection threshold of $\mu_{\text {mir }} \sim 0.032$. Within a simulation time of $\sim 10^{5} \Omega_{c}^{-1}$, we need $k I(k) \sim$ $10^{-5}$ for effective QLD. This is achieved for $k$ up to $k_{\text {cut }} \sim 10 k_{m}$, by looking at Figure 12 at $t=10^{5} \Omega_{c}^{-1}$. According to the same calculation as in Equation (44), we find mirror reflection can be achieved for particles with $p \gtrsim p_{\text {min }}=\left(k_{m} / k_{\text {cut }}\right) \mu_{\text {mir }}^{-1} \sim 3 p_{0}$. However, we see that by this time, particles with $p \gtrsim p_{0}$ are already fully isotropized, and lower-energy particles have also under- 

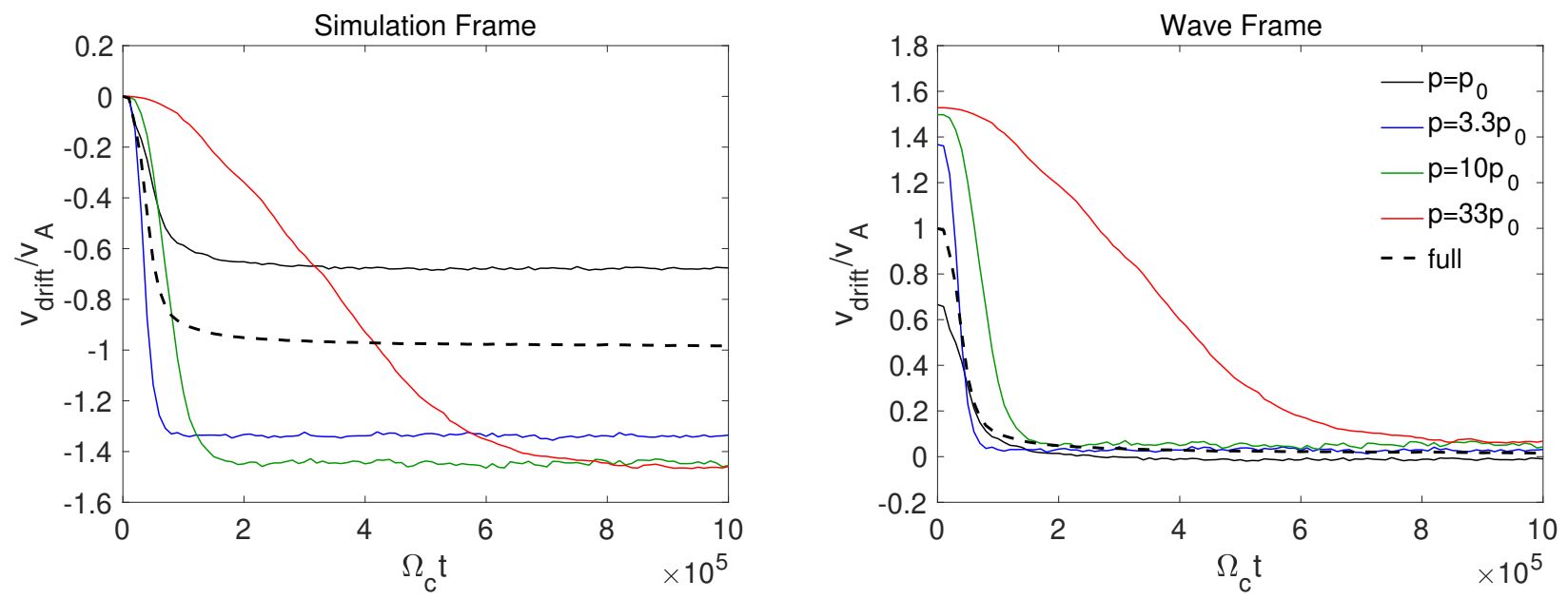

FIG. 13. - Same as Figure 10, but for run M3, showing evolution of mean particle drift velocity in different momentum bins, together with the full drift velocity (see legend).
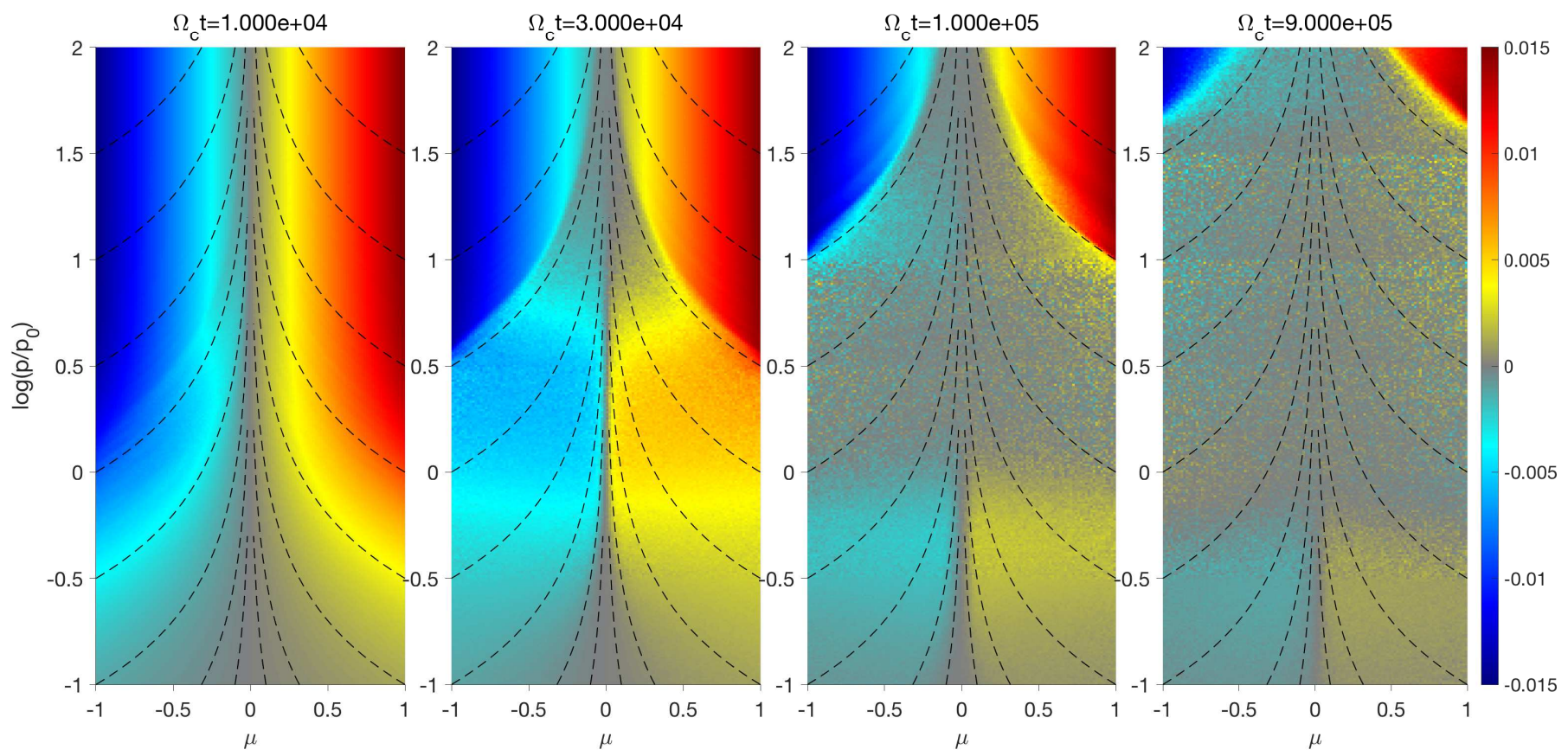

FIG. 14.- The 2D distribution function $\delta f_{w} / f_{0}$ in the wave frame for four snapshots from run M3. The dashed lines are contours in momentum space with the same resonance condition.

gone partial isotropization. Again, we conclude mirror reflection appears insufficient to explain the detailed evolution of the momentum distributions.

\subsection{Overcoming the $90^{\circ}$ Barrier}

To reveal the mechanism behind particle crossing the $90^{\circ}$ pitch angle in run M3, we have randomly selected a subsample of particles (1280 per energy bin), and closely followed their trajectories for a time interval of $200 \Omega_{c}^{-1}$ starting from $t_{0}=6 \times 10^{4} \Omega_{c}^{-1}$. We count the number of particles that have undergone reflection across $90^{\circ}$ over this interval. Here, we consider particle pitch angle in the wave frame that is relative to the full magnetic field vector (rather than background field), which is marked by $\mu_{w}^{\prime}$. To be counted, we require that $\mu_{w}^{\prime}$ before and after this time interval to be beyond $\pm v_{A} / \mathbb{C} \sim 0.0033$ (chosen somewhat arbitrarily, but this does not qualitatively affect the counting result). Among the 4 central momentum bins from $p=0.1 p_{0}$ to $10 p_{0}$, we find $9,13,20$, 23 particles are reflected. We looked at the trajectories of all these particles, and choose to show four particles with characteristic behaviors from each momentum bin in Figure 16.

\subsubsection{Representative Cases}

For the first particle with $p \approx 0.2 p_{0}$ that undergoes a reflection, we see that surprisingly, the field strength it experiences is almost constant. Quantitatively, the 

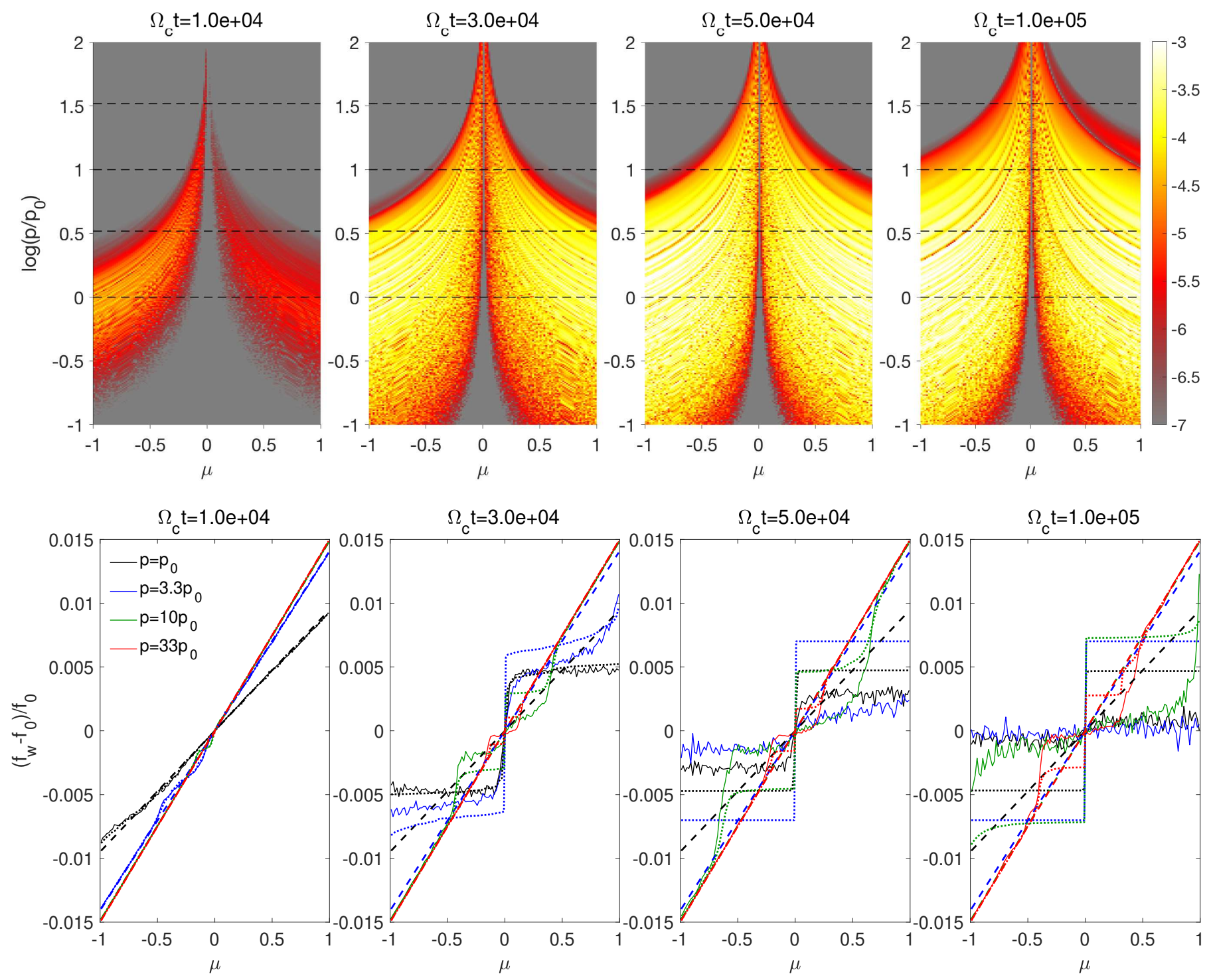

FIG. 15.- Same as Figure 9, but for run M3 at four different snapshots.

perpendicular field strength over this time interval is $\delta B \in(0.0398,0.0417)$. The range it spans translates to $\mu_{\text {mir }}=1.3 \times 10^{-3}$. While the particle does evolve quasi-adiabatically with many gyrations (which is typical for lower energy particles given their small gyro-radii and lack of power at small scales), its $\mu_{w}^{\prime}$ changes by a factor of several larger than the expected $\mu_{\mathrm{mir}}$. Checking the magnetic field profile in the bottom panel, we see that the particle is traveling against a shallow gradient in $B_{z}$. The range of $B_{z}$ experienced by the particle $\in(-0.081,-0.041)$, which changes the magnetic field orientation by $\sim 0.04$. While this is no proof that the particle must be reflected, it can well accommodate the change in particle $\mu_{w}^{\prime}$, which is less than $5 \times 10^{-3}$.

For the second particle with $p \approx 0.9 p_{0}$, its higher velocity allows it to traverse larger distances over the course of reflection, and experiences more rapid magnetic fluctuations. We have again checked that variations in total field strength are insufficient for mirror reflection. Re- flection occurs again when the particle travels against the gradient of individual field components, and in this time, we have $B_{y}$ increasing rapidly and $B_{z}$ decreasing more gradually. These variations change the magnetic field orientation by $\sim 0.05$ within just a few particle gyrations, which is again more than sufficient to account for the change in particle pitch angle by $\sim 0.02$.

In the remaining two examples, reflection occurs within two gyro-orbits. These cases are more common among relatively energetic particles: because of their larger gyro-radii, they more often experience abrupt changes in magnetic field. These changes average out to the leading order in quasi-linear theory, except when they lead to reflection. We see that the threshold pitch angle for reflection can be higher for these particles, and can reach as large as $\mu \sim 0.1$. This is accompanied by abrupt changes in $B_{y}$ and $B_{z}$ at comparable levels within the distance that the particle travels in one gyration.

\subsubsection{Non-linear Wave-Particle Interaction}



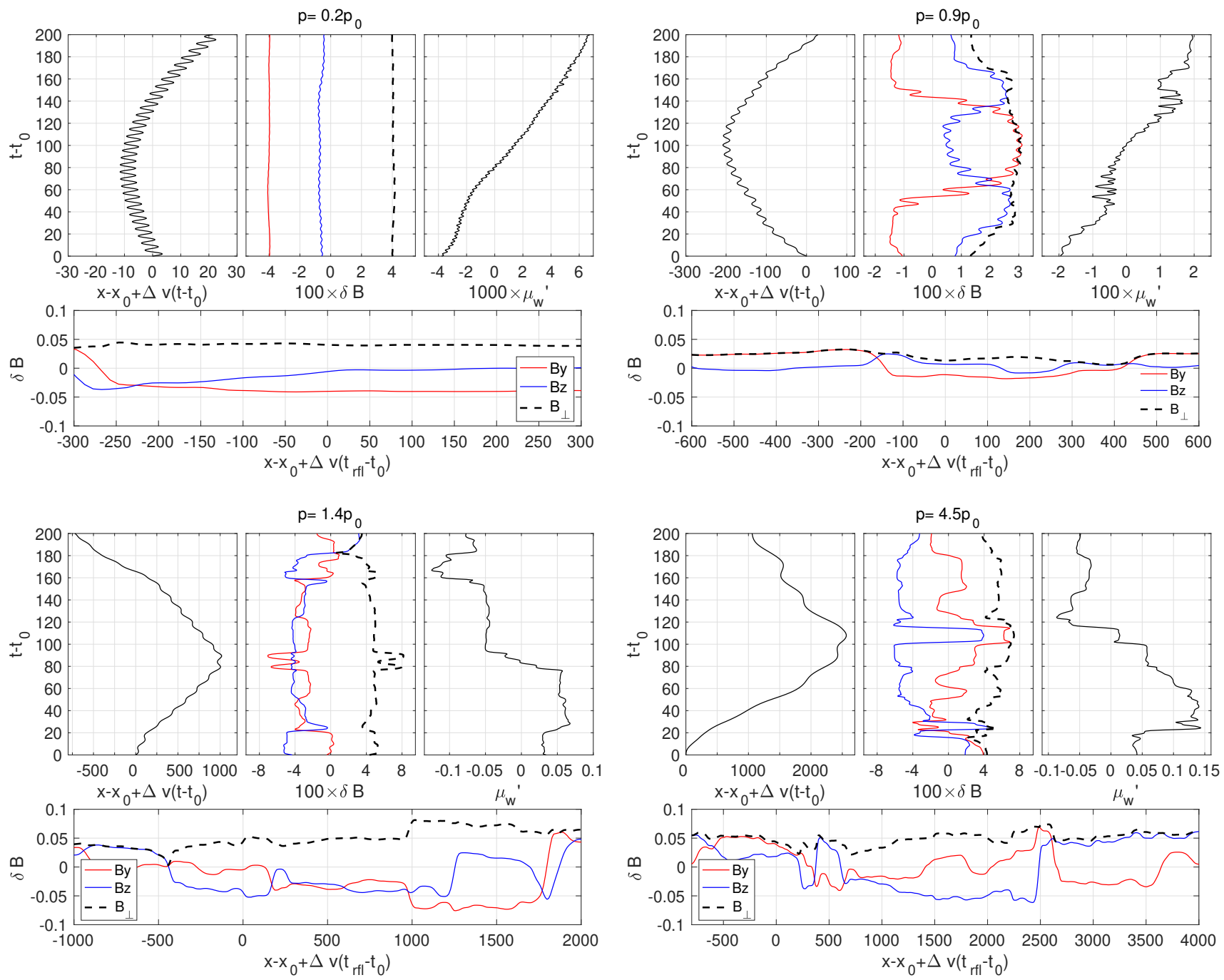

FiG. 16. - Four typical reflection events, for a particle with $p=0.23 p_{0}$ (top left), $p=0.92 p_{0}$ (top right), $p=1.39 p_{0}$ (bottom left), and $p=4.52 p_{0}$ (bottom right). They are selected right after time $t_{0}=6 \times 10^{4} \Omega_{c}^{-1}$. Among each group of plots, the top three panels show the time evolution of particle position in the wave frame, perpendicular field strength (individual components in color and total in black dashed) experienced by the particle, and its pitch angle cosine in the wave frame relative to the total field. The bottom panel shows the perturbed magnetic field profiles at the time of reflection.

Overall, the four examples roughly cover the range of behaviors of particles during reflection. They can be interpreted under the framework of non-linear waveparticle interaction (Völk 1973).

Reflection is most effective (almost instant) for the last two cases in Figure 16, for which abrupt changes in fields occur on a scale shorter than the distance that particle travels in one gyro time. In this regard, we can discuss the critical pitch angle below which non-linear wave-particle interaction takes over to reflect particles as follows. For a particle with momentum $p$ (and velocity $v$ ), let $\mu_{\text {crit }}$ be this critical pitch angle. It travels by $L_{\text {crit }} \sim 2 \pi v \mu_{\text {crit }} / \Omega$ in one gyro time, corresponding to a wave number $k_{\text {crit }}=\Omega /\left(v \mu_{\text {crit }}\right)=\Omega_{c} /\left(p \mu_{\text {crit }}\right)$. Reflection occurs when an abrupt change in magnetic field orientation is comparable to $\mu_{\text {crit }}$. Note this depends on the spectrum of waves at scales below some $\zeta\left(k_{\text {crit }}\right)^{-1}$, we arrive at a relation

$$
\mu_{\mathrm{crit}}^{2} \sim C \int_{k_{\mathrm{crit}} / \zeta}^{\infty} I(k) d k,
$$

where there is a square in the left hand side because $\mu_{\text {crit }} \sim \delta B / B_{0}$, while the right hand side corresponds to its square. We have also include dimensionless factors $\zeta$ and $C$ that encapsulate the roughness of the model.

The solution to this equation gives $\mu_{\text {crit }}$, which exhibits the following features

- Higher wave intensity leads to smaller $\mu_{\text {crit }}$, because more wave power at smaller scales makes reflection easier.

- Higher particle momentum leads to larger $\mu_{\text {crit }}$, because larger gyro scale accommodates more wave power to build up abrupt field profiles. 

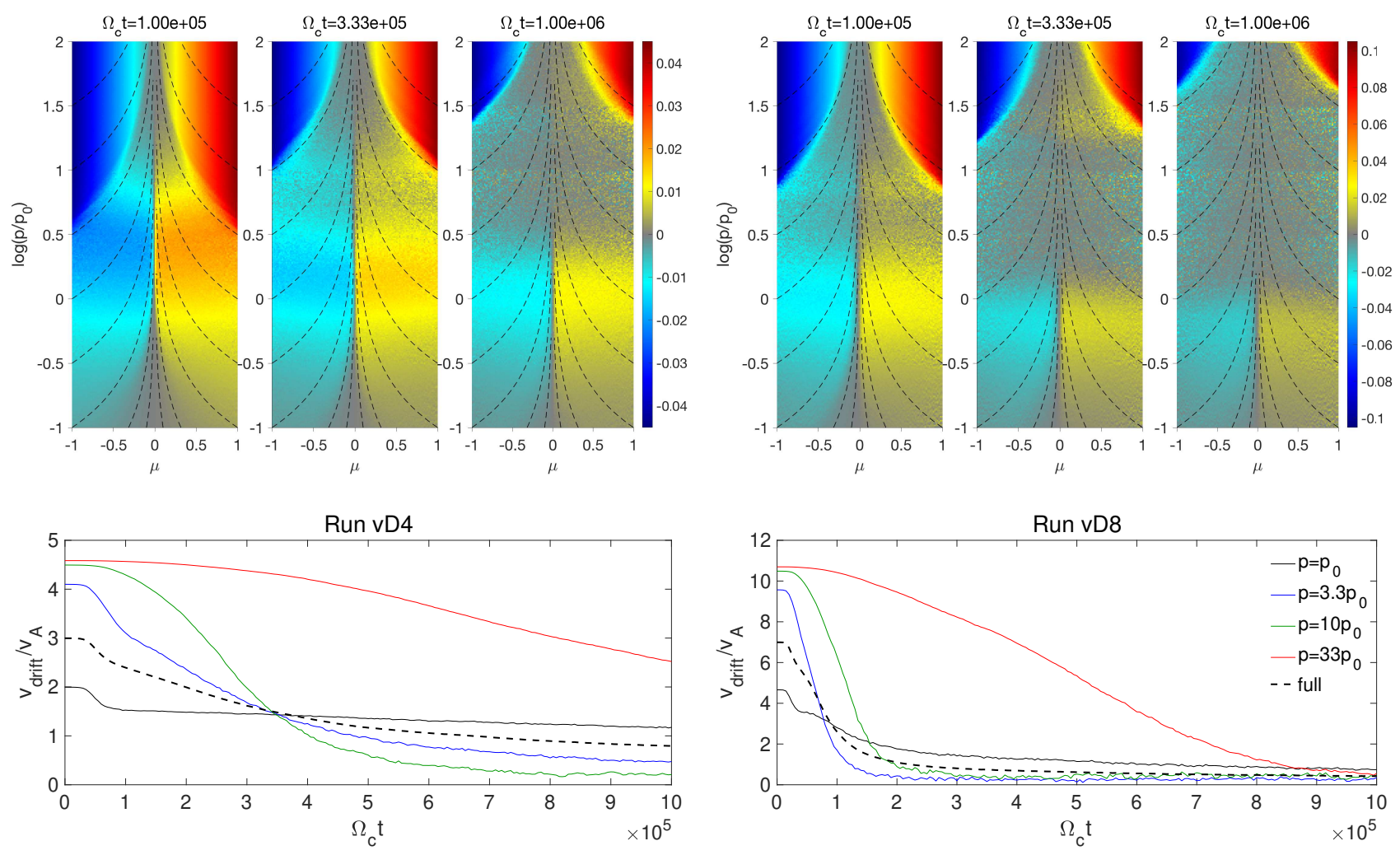

FIG. 17.- Distribution function and drift velocities for runs vD4 (left) and vD8 (right). Top two panels: $2 \mathrm{D}$ distribution function $\delta f_{w} / f_{0}$ in the wave frame at three snapshots. Dashed lines are contours in momentum space with the same resonance condition. Bottom two panels: time evolution of particle drift velocity in the wave frame for different momentum bins, as well as the full drift velocity (see legend).

- If there is a lack of power at small scales, there can be no solution, meaning that direct reflection in a couple gyro orbits is unlikely.

Clearly, all these features qualitatively agree with our findings. They are also justified through our discussions in Section 5.4.1.

To be more quantitative, we note that while the integral of $I(k)$ over $k$ gives the rms fluctuations, actual fluctuations can easily exceed the rms value, and deviations by up to $3 \sigma$ are not rare. We thus take $C \sim 3^{2}$ to account for the upper end of fluctuations. We may further take $\zeta \sim 4$ considering that waves with wavelengths up to $4 L_{\text {crit }}$ can contribute significantly to the local slope within this length scale of $L_{\text {crit }}$ (i.e., sinusoidal variations have roughly constant maximum slope in just $1 / 4$ of its periodic domain consecutively). With these numbers, and taking $p=4.5 p_{0}$ for run M3, we can derive $\mu_{\text {crit }} \sim 0.1$ (which gives $k_{\text {crit }} / \zeta \sim 1.8 \times 10^{-3}$, and the integral gives 0.0011$)$.

Equation (46) closely resembles Equation (54) of Völk (1973), with their $v_{\text {osc }} \sim 0.25-0.3\left(v \mu_{\text {crit }}\right)$, interpreted as the rms velocity oscillation amplitude of a particle in "the fluctuation field that contributes to its diffusion". The effective diffusion coefficient at $\mu=0$ is then given by $\sim 3 \Omega v_{\text {osc }}^{2}$.

The discussions above apply to instant reflection upon encountering abrupt field gradients. This corresponds to the $n=1$ term in Equation (48) of Völk (1973). As one lowers particle momentum, $\mu_{\text {crit }}$ decreases, and even- tually no solution can be found. This situation applies to lower-energy particles in Figure 16. More generally, higher order effects enter (corresponding to the $n>1$ terms), allowing particles to be reflected over multiple gyrations in a fluctuating field. Being less effective, it is consistent with fewer crossing particles found in our particle counting in lower-energy bins.

\section{SIMULATION RESULTS: PARAMETER STUDY}

Our fiducial run selects a parameter range that is physically more realistic but numerically challenging, with small $n_{\mathrm{CR}} / n_{i}$ and low CR drift velocity $v_{D}$, leading to relatively small growth rate and relatively low wave saturation level. In this Section, we vary parameters around our fiducial run, and conduct a two-part parameter study. In Section 6.1, we examine the saturation of the CRSI when $v_{D}$ is increased to higher values. In Section 6.2, we discuss results of varying solely numerical parameters for our run Fid.

\subsection{Dependence on Drift Speeds}

With $v_{D}$ increased to $4 v_{A}$ and $8 v_{A}$, we find that we recover the linear growth rates very well (not shown), with left and right handed modes growing at comparable rates (as expected). Figure 4 includes the time evolution of wave energy for runs vD4 and vD8 in dotted lines. They initially follow very similar evolutionary paths as our run Fid, except that everything (linear growth and QLD) proceeds faster. More notably, later evolution of these two runs are more similar to run M3, where wave 


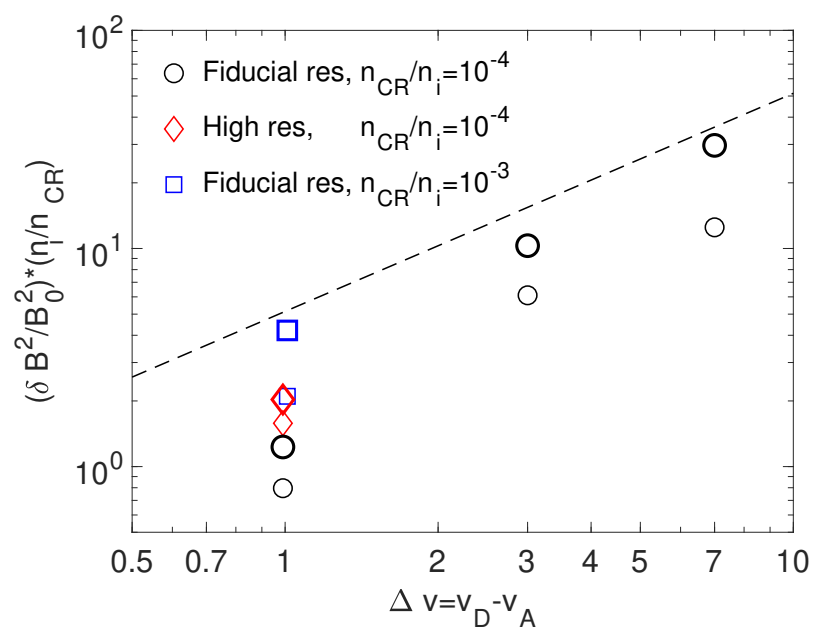

Fig. 18. - Saturation level measured from our simulations, in terms of wave energy density normalized by CR fraction. Black circles are from runs Fid, vD4 and vD8, red diamonds are from run Fid-hires, and blue squares are from run M3. Small/thin symbols are measured based on the total wave energy density, while large/thick symbols are measured from the same runs, but corrected from numerical dissipation. The dashed line indicates the expected saturation level assuming all CRs are fully isotropized, obtained by setting Equations (22) and (24) equal.

energy eventually plateaus, indicating saturation is close to completion.

In Figure 17, we show results related to quasi-linear evolution of runs vD4 and vD8. The overall outcomes are in between those of run Fid and run M3. Crossing across $90^{\circ}$ becomes easier as $v_{D}$ increases. By the end of the simulations, particles with $4 p_{0} \lesssim p \lesssim 20 p_{0}$ are roughly fully isotropized in run vD4. For run vD8, the range enlarges to $p_{0} \lesssim p \lesssim 40 p_{0} .{ }^{7}$ Lower-energy particles are in the process of (but have not yet completed) isotropization. Isotropization is limited by the rate of $90^{\circ}$ crossing, which is much slower for these lower-energy particles (see discussion in Section 5.4).

\subsubsection{The Saturation Level}

At this point, we can gather all simulation runs discussed so far to address the saturation level of the CRSI as a function of $v_{D}$. The saturation level is measured in terms of wave energy density. With equipartition of magnetic and kinetic energies, it can simply be represented by $\left\langle\delta B^{2} / B_{0}^{2}\right\rangle$ in dimensionless form. Theoretically, the predicted wave energy saturation level is obtained by equating Equations (22) and (24), and is proportional to $\left(n_{\mathrm{CR}} / n_{i}\right) \Delta v$. Integrating the derivative of the distribution function numerically, the result is shown as the dashed line in Figure 18.

The final wave energy density obtained from our runs Fid, vD4, vD8, M3, and Fid-hires (discussed in the next

7 Two numerical artifacts can be identified in Figure 17. The banded horizontal feature in the top panels is due to the division into 8 particle momentum bins. Within each bin, there is more noise towards higher energies because there are fewer particles. One may also find that particle mean drift velocities at various energies do not reach zero even they are theoretically expected to be fully isotropized (bottom panel). This is related to coarse particle binning during outputs (mentioned earlier in Section 5.3.2).

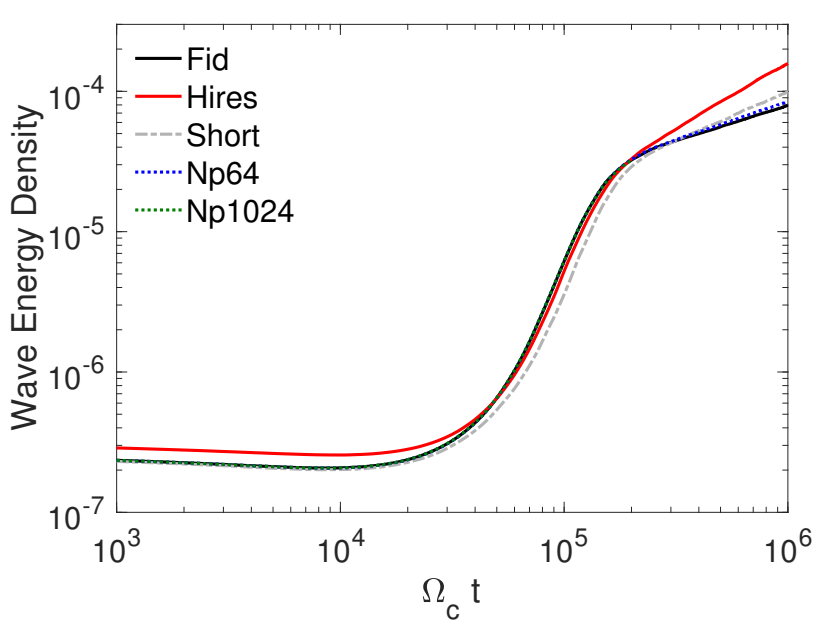

FIG. 19.- Time evolution of total wave intensity for runs with different numerical parameters.

subsection) are also shown in Figure 18. Even though runs $\mathrm{M} 3$ and vD8 are close to complete saturation, their final wave energy densities appear to be far from the expected level. This is because numerical dissipation, while very low in the Athena MHD code, is still non-negligible for the extremely long simulation time necessary to reach saturation. 8 With an adiabatic equation of state, we can capture the change in gas internal energy over time (thanks to the excellent energy conservation properties in the gas component), and find that it increases steadily as result of numerical dissipation, and reaches a level that is comparable to the overall wave energy density by the end of the runs. When we add this energy lost to dissipation to the direct wave energy, we find much more reasonable saturation levels, as shown in thick/larger symbols in Figure 18. In particular, run Fid remains at a level below the full saturation prediction because particles become "stuck" at $90^{\circ}$, whereas runs vD4 and vD8 are more and more close to the expected saturation level, as more particles are fully isotropized. Run M3, which based on its distribution function is clearly isotropized, is excellent agreement with theoretical expectations for the wave amplitude.

\subsection{Numerical Parameters}

Here, we examine our runs Fid-Hires, Fid-short, FidNp64 and Fid-Np1024, in which we keep physical parameters the same and vary only numerical parameters (note that for run Fid-Hires, we have slightly reduced the box size to save computational cost). In Figure 19, we show the time evolution of the wave energy density of these runs. Overall, these three runs proceed very similarly to run Fid. ${ }^{9}$ QLD proceeds slightly slower in run FidShort, but it catches up to the other runs shortly after. Run Fid-Hires eventually grows to reach larger wave amplitudes, which we will show later is due to more efficient

8 Dissipation of low-amplitude waves imposed in the initial conditions is also evident at early times in Figure 4.

9 All these runs have the same initial wave amplitude $A=10^{-4}$ (see Equation 42). Run Fid-Hires thus has more initial wave energy because of more spectral range it covers. 


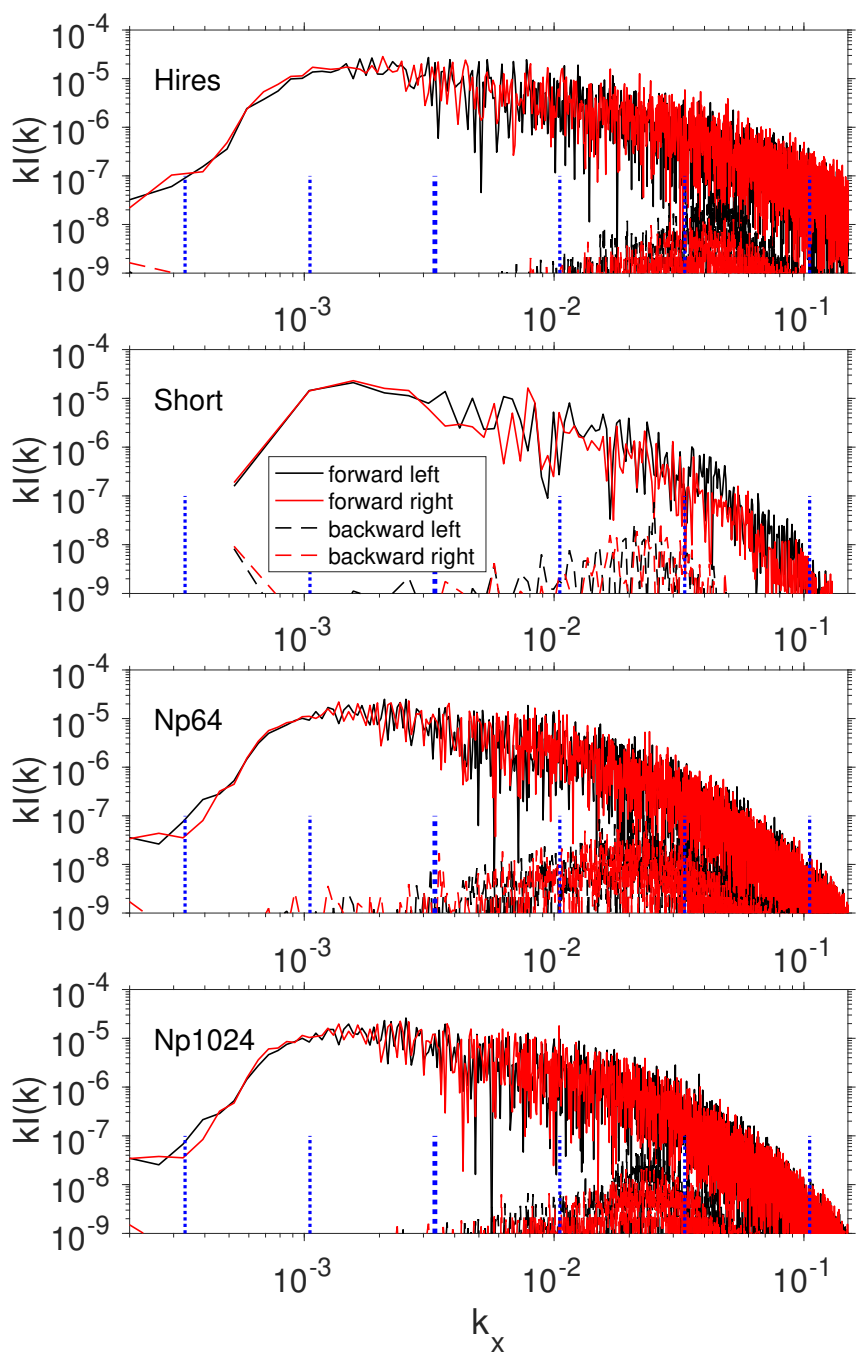

FIG. 20.- Wave spectrum at time $t=2 \times 10^{5} \Omega_{c}^{-1}$ from runs Fid-hires, Fid-Short, Fid-Np64 and Fid-Np1024. Vertical dotted lines are identical to those in Figure 8 (the location of the thick line indicates the most unstable wavenumber).

crossing of $90^{\circ}$ pitch angles.

In Figure 20, we show the wave spectrum from these runs at time $t=2 \times 10^{5} \Omega_{c}^{-1}$. The overall shape of the spectra are comparable to each other. The highresolution run has less numerical dissipation, allowing the wave spectra to extend to larger $k$. Run Fid-short has a box size that is only $1 / 3$ of the fiducial box (but still long enough to fit $\sim 17$ most unstable modes), and hence modes are a lot more sparsely sampled, especially at low$k$. This is likely related to its initially slower quasi-linear evolution. We have also tried simulations with $1 / 8$ the fiducial box size (not shown); while evolution is smooth, the lack of power at larger scales start to adversely affect the evolution of higher-energy particles.

Figure 20 shows that there are backward-propagating modes excited in all these runs. The amplitude of these modes reflects the level of noise. Clearly, inserting more particles helps reduce the noise. The bottom line for properly choosing particle number is that the amplitude of such backward-propagating modes should always be much smaller than the forward-propagating modes driven by the CRSI. In this regard, our run Fid-Np64 is acceptable, but more caution should be exercised if one were to use even fewer particles.

In Figure 21, we show the particle distribution functions in the wave frame at time $t=10^{5}$. These may be compared with Figure 7. Overall, runs Fid-short and Fid-Np64 are more noisy, whereas run Fid-Np1024 appears exceptionally smooth. Our run Fid lies in between. Despite these differences in noise levels, subsequent QLD does not appear to be affected.

Of particular interest is the high-resolution run FidHires, where waves grow to substantially higher amplitudes. In Figure 22, we show three snapshots of the particle distribution function in the wave frame for four representative momentum bins. These frames are to be contrasted with Figure 9 for the same snapshots. Clearly, in this run, a substantial fraction of particles with $p \gtrsim 3 p_{0}$ have managed to cross the $90^{\circ}$ barrier. This fraction steadily increases with time and, were we to run the simulation longer (e.g., to $t=2 \times 10^{6}$ ), particles in these momentum bins would essentially be fully isotropized. This trend can also be seen from the last panel, which shows the time evolution of the mean CR drift velocity: $v_{d}$ for particles with $p \gtrsim 3 p_{0}$ are steadily decreasing towards zero. Clearly, crossing the $90^{\circ}$ barrier is easier compared to run Fid. This is natural because higher resolution allows more small-scale modes to be better preserved, thus enhancing the chance of non-linear wave-particle interaction to reflect the particles (see Section 5.4.2). In Figure 18 , we see that the wave energy density in our run FidHires is also a factor of several closer to the expected saturation level. We expect that increasing the resolution further (which would be much more computationally costly) would eventually lead to full isotropization of the bulk CR populations.

In sum, our exploration of numerical parameters suggests that full saturation of the CRSI (ideal case, no damping) can be achieved as long as there is sufficient numerical resolution. The required resolution is mainly limited by the demands imposed by crossing the $90^{\circ}$ pitch angle, which is sensitive to wave amplitudes at small scales. Similar results to our fiducial model can be achieved when one reduces the number of particles and/or the box size at some modest level ( $\sim$ a factor of $\sim 3$ ).

\section{DISCUSSION}

\subsection{CR Feedback to Background Gas}

Through the CRSI and QLD, CRs transfer momentum to background gas, which serves as an important dynamical feedback mechanism in broad range of astrophysical environments. In the following, we take run vD8 as an example and discuss CR feedback in terms of momentum and energy.

Momentum feedback is manifested in the top panel of Figure 23, which shows changes in the mean gas and CR momentum density $\left(\Delta \mathcal{P}_{g}\right.$ and $\left.\Delta \mathcal{P}_{\mathrm{CR}}\right)$. In our simulation frame, the initial CR momentum is zero, and initial gas momentum is negative. Over time, the CR momentum density decreases, accompanied by an increase in gas mo- 

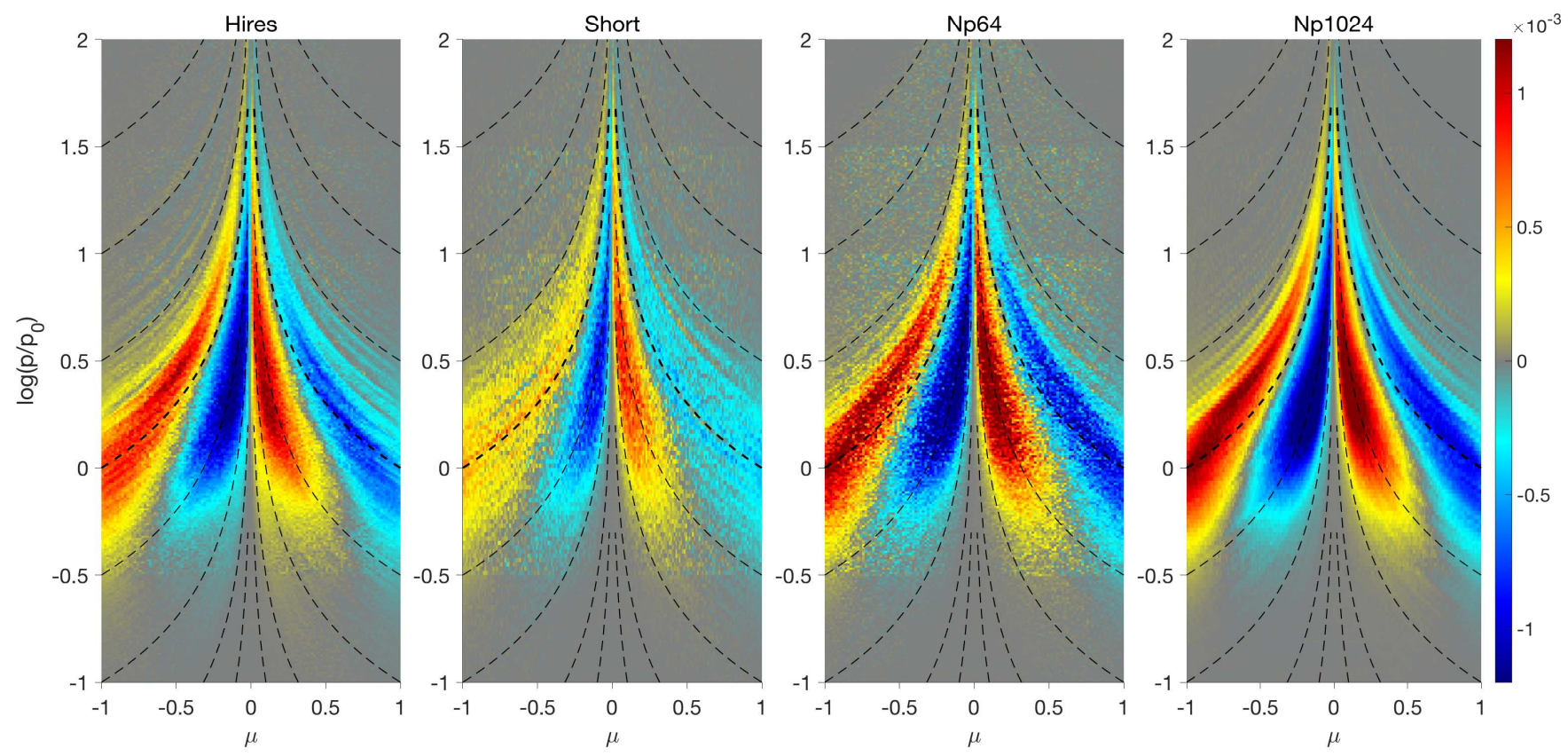

Fig. 21. - The 2D distribution function $\delta f / f_{0}$ in the lab (simulation) frame at time $t=10^{5} \Omega_{c}^{-1}$ for runs Fid-Hires, Fid-Short, Fid-Np64 and Fid-Np1024, respectively. The dashed lines are contours in momentum space that are resonant with the same wave $(p \mu=$ const $)$, with the thick line marking $p \mu=p_{0}$. The color limits are identical among the four panels.
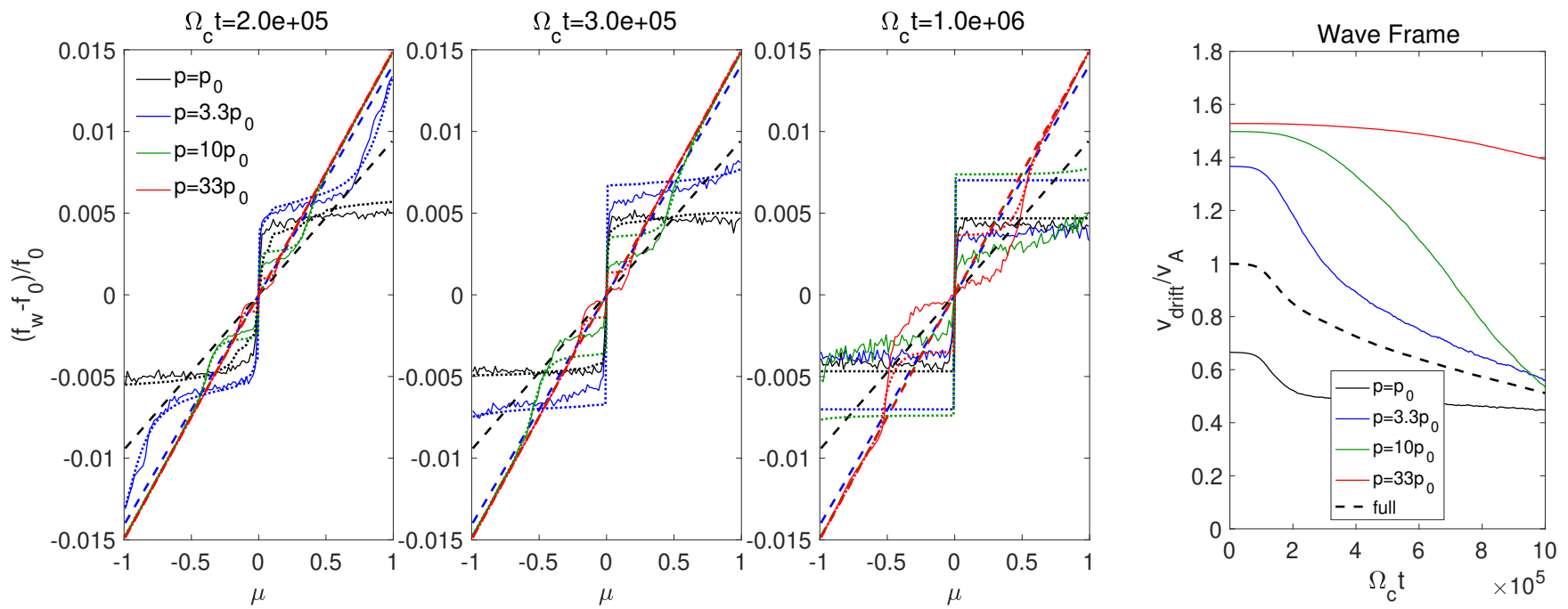

FIG. 22.- Results from run Fid-Hires. Left three panels: snapshots of particle distribution function in the wave frame $\left(f_{w}-f_{0}\right) / f_{0}$ for four representative momenta as a function of pitch angle cosine $\mu_{w} \approx \mu$. Solid lines are measured from the simulations, thick dashed lines indicate the initial distribution, while the dotted lines are reconstructed distribution functions obtained by solving the QLD Equation (14) in time. Right panel: time evolution of particle drift velocity in the same four momentum bins in the wave frame (solid lines). The thick dashed line represents the mean drift velocity averaged over the entire particle population.

mentum density of exactly the same amount. Physically, the backreaction from the $\mathrm{CR}$ isotropization process effectively gives rise to a "parallel force" $F_{\|}$. This parallel force is mediated by forward-propagating Alfvén waves, and transfers CR momentum to the gas (e.g., Thomas \& Pfrommer 2018). The amount of momentum transferred to the gas is in fact the same as the "effective" wave momentum defined in Equation (24), which is the momentum needed to excite such forward-propagating waves (Kulsrud 2005). By the end of our run vD8, al- though the evolution of gas and CR momenta are not yet fully saturated, both are approaching the expected saturation level indicated by the dashed lines, obtained from integrating Equation (22). In the case of run M3 (not shown here), we do observe that saturation of gas and $\mathrm{CR}$ momenta is achieved. That is, the mean CR streaming velocity relative to the gas is reduced from $2 v_{A}$ to $v_{A}$ (Figure 13 shows that the final mean $\mathrm{CR}$ velocity is zero in the wave frame), and the gas momentum density is increased to balance the loss from CRs. 

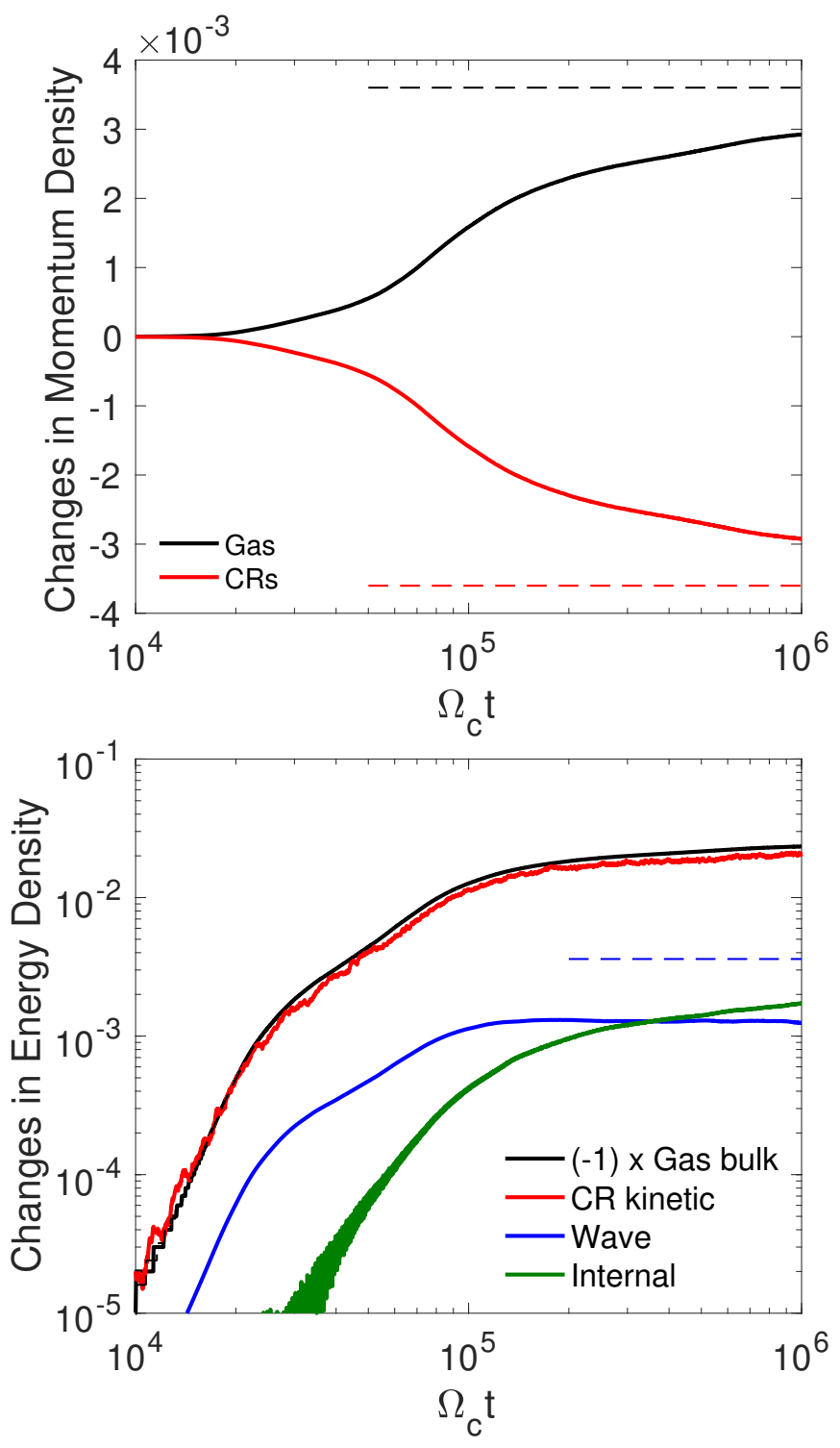

FIG. 23. - Time evolution of changes in momentum density (top) and energy density (bottom) in various components (labeled in the legends) in run vD8. The horizontal dashed lines mark the expected saturation levels (top: momenta; bottom: wave energy). See Section 7.1 for more details.

Energy feedback can be discussed by decomposing the energy budget into various components. These include gas bulk kinetic energy $E_{k \text {,bulk }}=\left\langle\rho v_{x}^{2} / 2\right\rangle$, representing gas motion parallel to background field, wave energy $E_{\text {wave }}, C R$ kinetic energy $E_{\mathrm{CR}}$, and internal energy of the gas $E_{\text {internal }}=P_{g} /(\Gamma-1)$. The time evolution of these quantities is shown in the bottom panel of Figure 23 , where we only plot their changes relative to the initial condition. The overall energy conservation is expressed as

$$
E_{k, \text { bulk }}+E_{\mathrm{CR}}+E_{\text {wave }}+E_{\text {internal }}=\text { const } .
$$

Note that $E_{k \text {,bulk }}$ and $E_{\mathrm{CR}}$ are frame-dependent. Here it suffices to report results in our simulation frame.

In the simulation frame, the gas bulk energy density decreases over time (as the bulk gas velocity becomes less negative). This is associated by the work done by the same parallel force $F_{\|}$that changes the gas momentum. In fact, we can exactly reproduce the black solid line in the bottom panel of Figure 23 using $F_{\|}$inferred from acceleration in the gas (from the top panel), with $\Delta E_{k, \text { bulk }} \approx v_{g} \Delta \mathcal{P}_{g}$. (Since $v_{g}$ only changes by a tiny fraction over the process, it is approximately taken to be constant here.) This same force also does opposite work on the CRs, increasing their total kinetic energy. Some of this energy is used to excite and amplify the waves, leading to their continued growth. In the mean time, the waves are slowly dissipated numerically, increasing the internal energy of the gas. ${ }^{10}$

We emphasize that the CRSI itself does not lead to heating of background gas. Heating only occurs through internal dissipation in the gas, which is generally accompanied by wave damping. This fact is reflected in the more recent formulation of CR-(magneto)hydrodynamics of Thomas \& Pfrommer (2018), though not necessarily correctly modeled in some earlier studies. If wave growth is balanced by wave damping in a quasi-steady state (not achieved in our present simulations), then the heating rate is simply determined by the rate of wave growth/damping.

\subsection{Towards More Realistic Simulations}

There are several directions to further improve the realism of our simulations. In particular, there are several wave damping mechanisms to be incorporated, including ion-neutral damping, non-linear Landau damping, and turbulent damping of waves.

Ion-neutral damping, or ampibolar damping, is well known (e.g., Kulsrud \& Pearce 1969; Soler et al. 2016). It is present when background gas is partially ionized. The ions are directly coupled and respond to magnetic fields, but the neutrals hardly feel the magnetic fields and can possess different bulk velocity from the ions. Ion-neutral collisions give friction, and lead to damping of Alfvén waves in the ion fluid, especially at small scales. Given that crossing the $\mu=0$ barrier is sensitive to the spectrum of waves at small scales, ambipolar damping may cause severe challenge to the isotropization of the CRs. This effect will be incorporated and studied in greater detail in a follow-up paper.

Non-linear Landau damping is a result of wave-particle interaction at Landau resonance between background thermal particles and a beat wave formed by two propagating waves (Lee \& Völk 1973), and is more effective in high- $\beta$ plasmas. Our MHD-PIC formulation do not capture the kinetic physics of the background plasma, and hence can not capture non-linear Landau damping. However, this is not necessarily a limitation: we would lose the advantage of MHD-PIC by resolving the kinetic physics, and in principle, this damping mechanism can be incorporated in the form of sub-grid physics.

${ }^{10}$ While damping in our simulations is purely numerical, we can see that it is negligible in early phases of linear and quasi-linear evolution. Only towards later stages where saturation is largely achieved (and hence there is little wave growth) does numerical dissipation become more noticible. Thus, our results are largely unaffected by numerical dissipation. 
Turbulent damping is due to the distortion of CRgenerated Alfvén waves when they collide with oppositely directed wave packets originating from background turbulence. As a result, the wave energy cascades into smaller scales and is ultimately dissipated (Farmer \& Goldreich 2004). This damping rate has only been calculated approximately (e.g., see more recent work of Lazarian 2016). Incorporating this effect is fully compatible with our MHD-PIC framework, and can be studied in future multi-dimensional simulations.

For this first study, we focused on the classical theory with 1D simulations with uniform background density and magnetic field, which enable us to confirm resolution and box size requirements. Multi-dimensional extensions are straightforward and are underway, although are much more computationally expensive. Such extensions would allow modes propagating obliquely with respect to background field to be investigated, and open up more parameter space, as the relative importance of thermal, magnetic and CR pressures, and background turbulence can play certain roles,

Closely related to the CRSI is another type of gyroresonance instability, which is driven by $\mathrm{CR}$ pressure anisotropy (e.g., Lazarian \& Beresnyak 2006), and can be easily triggered in compressible MHD flows (when CR pressure anisotropy exceeds $\sim v_{A} / c$ ). It excites circularly polarized Alfvén waves propagating to opposite directions, which then isotropize the CRs via QLD. Recently, Lebiga et al. (2018) studied this instability numerically based on the MHD-PIC framework in full $f$ approach (with relatively strong anisotropy), and found good agreement with quasi-linear theory. In the absence of CR streaming, the $\mathrm{CR}$ distribution function is always symmetric about $\mu=0$, and hence crossing $90^{\circ}$ pitch angle is not required for $\mathrm{CR}$ isotropization. In reality, $\mathrm{CR}$ streaming and pressure anisotropy likely co-exist. This would excite a wide ranges of waves propagating in both directions, calling for further investigations.

Finally, in any simulation with periodic boundary conditions (even with arbitrarily high resolution such that crossing $90^{\circ}$ pitch angle is not a limitation), there is a finite free energy of the system based on the initial energy and momentum densities of gas and CRs. In the absence of damping (numerical or physical), growth of Alfvén waves would halt when QLD has reduced the mean velocity difference between the gas and the CRs to $v_{A}$. In reality, at a given location the CRSI is typically driven by a large-scale CR pressure gradient. For instance, CRs are continuously generated from the galactic midplane region, and diffusively propagate downhill through the gradient to escape from the galaxy. Energy and momentum are continually added to the system. This configuration likely yields continued wave growth, balanced by damping to reach a steady state, a situation that has yet to be addressed with more realistic boundary conditions.

\subsection{Comparison with conventional PIC simulations}

Our MHD-PIC approach to study the CRSI strongly contrasts with conventional full-PIC or hybrid-PIC approaches that are widely used in modeling highly nonlinear plasma problems. The full-PIC approach treats charged particles of all kinds as particles. It resolves all scales down to the electron skin depth (eventually the Debye length), and hence all plasma wavemodes are self-consistently captured along with relevant waveparticle interactions. The hybrid-PIC approach treats electrons as a massless conducting fluid. By compromising the electron-scale physics, it alleviates the stringent resolution requirement of the full-PIC method, while it can still capture the ion-scale physics well. For the CRSI problem, employing full-PIC or hybrid-PIC methods means that background ISM fluid must be represented by particles, hence resolving the corresponding microscopic scales. This causes two major drawbacks: substantially enhanced numerical cost, and PIC noise due to the finite number of particles representing the background plasma.

As already discussed in Section 3, there is substantial scale separation in the CRSI problem between the CR gyro-radii and the ion skin depth (and below). Conducting a hybrid-PIC simulation using the same parameter settings as our fiducial simulations would require more than an order of magnitude more cells, and another order of magnitude more cells would be needed in the full-PIC case. The numerical cost in the full-PIC case is further exaggerated by the ion-to-electron mass ratio, requiring a factor of $m_{i} / m_{e}$ more timesteps per CR gyro period. Moreover, the number of background particles per cell needs to be sufficiently high in order to reduce the noise floor as compared to the signal level of growing waves and avoid artificial QLD of the CRs by such noise.

Despite these difficulties, Holcomb \& Spitkovsky (2018) have recently presented a 1D study of the CRSI using full-PIC simulations. Typical parameters of their simulations are: $m_{i} / m_{e}=100, v_{A} / c=0.1$ (note in full-PIC, $c=\mathbb{C}$ in our notation), 10/100 cells per electron/ion skin depth, $N_{\mathrm{ppc}}=50-1000$ (all plasma particles equally divided among background and CR species), $n_{\mathrm{CR}} / n_{i} \in\left[2 \times 10^{-4}, 2 \times 10^{-2}\right]$ and $v_{D} / v_{A}$ from 1.4 (lowanisotropy) to 7.9 (high-anisotropy) ${ }^{11}$. They considered two different $\mathrm{CR}$ distribution functions: ring and powerlaw, with the former representing a mono-energetic CR distribution in the drift frame. They have successfully reproduced the peak growth rate in runs with the ring distribution, and similar to our results, achieved full isotropization in runs with relatively large $n_{\mathrm{CR}} / n_{i}$ and/or CR drift velocity.

We note that the large ratio of $v_{A} / c$ adopted in Holcomb \& Spitkovsky (2018) makes the resonant condition (2) asymmetric with respect to forward and backward traveling particles, thus modifying the standard CRSI dispersion relation for left/right handed modes in different ways. Together with the choices of large $n_{\mathrm{CR}} / n_{i}$ ratio, drift velocity being mildly relativistic, and the use of a ring distribution, these parameters represent substantial compromises to alleviate the issue of scale separation and particle noise. As a result, reasonable agreement with linear theory was found mainly in the special case of the ring distribution, and power-law distribution with exaggerated high anisotropy and high $n_{\mathrm{CR}} / n_{i}$

${ }^{11}$ Let us recall that in present study (Fid run) the parameters are: $m_{i} / m_{e} \rightarrow \infty, v_{A} / \mathbb{C}=3.3 \times 10^{-3}, 10$ ion skin depths per cell, $N_{\mathrm{ppc}}=2048$ (CRs only), $n_{\mathrm{CR}} / n_{i}=10^{-4}$ and $v_{D} / v_{A}=2$. 
values. These more extreme run parameters (approaching the Bell regime), together with the asymmetry in left/right handed modes caused by large $v_{A} / c$, make the left handed modes in these simulations grow at much reduced rates compared with right handed modes, leading to asymmetric scattering for forward/backward traveling CRs. While this situation might apply near strong shocks where the Bell instability operates, the parameter regime is not representative of typical conditions in the ISM. Additionally, they have suggested mirror reflection as being responsible for crossing $90^{\circ}$ pitch angle. However, the reflection event shown in their Figure 10 in fact appears more consistent with non-linear wave-particle interaction, as we have demonstrated in this work.

Perhaps the chief advantage of full-PIC and hybridPIC methods over the MHD-PIC approach is the ability to capture non-linear Landau damping, as the background plasma is treated kinetically. However, the physics of non-linear Landau damping is highly subtle, and damping relies on second-order longitudinal electric fields resulting from a beat wave formed from two circularly polarized Alfvén waves (Lee \& Völk 1973). It is conceivable that capturing such effect must require extremely low level of background noise, and hence exceedingly large number of background plasma particles: another major challenge yet to be resolved.

Finally, we comment that several of the difficulties for simulating the CRSI outlined in Section 3 are common for all variations of PIC methods. The $\delta f$ approach coupled with a $\kappa$ distribution that we have adopted could also be incorporated in full-PIC and hybrid-PIC simulations, likely leading to substantial improvements in future studies of the CRSI.

\section{SUMMARY AND CONCLUSIONS}

In this paper, we have presented the first numerical studies of the CRSI using the MHD-PIC method. We use 1D simulations in a periodic box and an idealized setup (no damping, a reduced speed of light $\mathbb{C}$ ), for a range of model and numerical parameters. We have been able to accurately reproduce the theoretically predicted linear growth rate, follow quasi-linear evolution as CR particles interact with CSRI-driven Alfvén waves, and identify the mechanism responsible for $\mathrm{CR}$ particles to cross the $90^{\circ}$ pitch angle. In the course of this study, we have developed techniques to investigate CRSI and QLD with substantial precision, paving way for future studies with more realistic setups and incorporating more physics.

Unlike conventional PIC methods, the MHD-PIC method has unique advantages in bypassing the microscopic scales of the background thermal plasma, allowing one to focus on the interaction between the CRs and background gas around the gyro-resonant scale. However, the CRSI still involves substantial scale separation, ranging from scales much smaller than, to scales much larger than, the CR gyro-resonant scale. Covering this large range of scales is necessary to properly capture the crossing of the $90^{\circ}$ pitch angle and QLD. Moreover, the extremely weak level of anisotropy $\left(\sim v_{A} / c\right)$ in the CR distribution function over a wide energy range makes it challenging to properly represent with a sufficiently large number of particles.

To address these challenges, we have extended the MHD-PIC formulation to use a $\delta f$ (instead of full $f$ ) representation of the $\mathrm{CR}$ particles on top of a known initial equilibrium distribution function $f_{0}$, which dramatically reduces the Poisson noise. While our fiducial simulations employ as many as 2048 particles per cell to achieve better precision, similar results can be achieved with fewer particles, and we recommend testing the level of Poisson noise in a non-CR-streaming simulation to determine the optimal number of particles. Using the conventional full $f$ representation, while the signature of the CRSI can be identified, the high noise level precludes any quantitative measurement of its overall properties.

The $\delta f$ method requires the background distribution function $f_{0}$ to be continuous, which is incompatible with $f_{0}$ being a truncated power-law. We have therefore chosen $f_{0}$ to be a $\kappa$ distribution, and the corresponding dispersion relation of the CRSI is analytically derived. We note that under realistic large-scale ISM conditions $\left(n_{\mathrm{CR}} / n_{i}\right.$ extremely small and moderate $\left.v_{D} / v_{A}\right)$, the left and right handed Alfvén waves grow at equal rates. The Bell-type modes, in which the right-handed modes grow faster, emerge when $n_{\mathrm{CR}} / n_{i}$ and/or $v_{D} / v_{A}$ become large. We have carefully chosen parameters to ensure sufficient scale separation $v_{A} / \mathbb{C}=1 / 300$, and that the left and right handed modes growth at about the same rates (except for run M3 where there is a small difference), so that the conditions are as close to ISM as possible. Our particle population also covers a wide range of $\mathrm{CR}$ momenta from $0.01 p_{0}$ to $100 p_{0}$, with $p_{0}=\mathbb{C}$ being the characteristic momentum of the $\kappa$ distribution.

We have found that when employing a standard periodic boundary condition, properly capturing the linear growth rate requires an excessively long simulation box, corresponding to the box-crossing time of a typical particle approaching the wave growth time. We attribute this to the fact that the growth of the CRSI is driven by QLD, which requires particles to encounter sufficient number of independent wave packets, and is related to the random-phase approximation commonly employed in describing QLD. To address this issue, we implemented a phase-randomization technique for particles that cross the periodic boundaries, which allows us to achieve even better results using much smaller simulation boxes (size $\lesssim 50$ instead of $\gtrsim 1000$ most unstable wavelength).

Equipped with these techniques, we are able not only to accurately reproduce predicted CRSI linear growth rates over a broad range of wave spectrum, but also to accurately follow the quasi-linear evolution of the system. We observe that QLD proceeds to isotropize the $\mathrm{CR}$ distribution function $f_{w}$ in the frame of forwardpropagating Alfvén waves, so that $\partial f_{w} / \partial \mu_{w}$ approaches 0 on either side of the pitch angle cosine $\mu_{w} \approx \mu=0$. With an increasingly flat distribution of particle pitch angles, wave growth slows down and eventually stops. As longer-wavelength modes that resonate with higherenergy CRs grow more slowly, wave growth proceeds from the most unstable to longer wavelengths (and in principle also to shorter wavelengths, though we lack resolution for these). In parallel, quasi-linear evolution 
proceeds from low-energy to higher-energy CRs as waves become available.

Whether full isotropization of CRs in the wave frame is achieved depends on whether particles manage to overcome the $\mu=0$ barrier (crossing the $90^{\circ}$ pitch angle). The mechanisms involved go beyond quasi-linear theory. While mirror reflection is commonly invoked as the main mechanism enabling crossing $\mu=0$, we have found that reflection generally occurs via non-linear wave-particle interaction, with particles encountering abrupt changes in field orientation. Reflection is more easily achieved for higher-energy particles, and is facilitated by the presence of waves at wavelength much smaller than the gyroresonance scale (see Section 5.4 for details).

In our study, efficient crossing of the $\mu=0$ barrier is achieved in simulation runs with strong wave growth (runs M3 and vD8). For these runs, full saturation is reached, with the mean $\mathrm{CR}$ drift velocity reduced to Alfvén speed. The final particle distribution function, as well as saturation amplitudes of the waves, are in excellent agreement with theoretical expectations. Our fiducial run, with a relatively low $n_{\mathrm{CR}} / n_{i}=10^{-4}$ and small initial drift speed $v_{D}=2 v_{A}$, becomes stuck in a state of pre-mature saturation: while the distribution is flat on each side of $\mu=0$ within most momentum bins, the bulk of the CR population fails to overcome the $\mu=0$ barrier. The inability of particles to breach the $\mu=0$ barrier is primarily due to numerical dissipation of lowamplitude waves at small scales, together with absence of even shorter-wavelength waves due to limited resolution. We have further found that enhancing numerical resolution can substantially alleviate the situation. Thus, proper choice of numerical resolution for given physical parameters is also crucial for saturation.

The CRs provide direct momentum feedback to background gas, which accelerates in accordance with momentum conservation of the gas-CR system. Wave power is directly proportional to the momentum transferred from the CRs to the gas. In terms of energy feedback, the work done by the CRs leads to gas acceleration and wave growth, but no direct heating. Gas heating is only achieved through wave damping, which is purely numerical in our simulations.

As a first study, we have restricted ourselves to $1 \mathrm{D}$ simulations in a periodic box in the ideal MHD limit. Our explorations have identified and clarified the numerical methods necessary to properly capture the essential mechanisms of the CRSI, and have paved the way for future investigations to incorporate more realistic physics. These may include implementing various damping mechanisms, extensions to multi-dimensions, and employing more realistic boundary conditions.

We thank Cole Holcomb, Matt Kunz, Peng Oh, and Anatoly Spitkovsky for useful discussions. XNB acknowledges support by the Youth Thousand Talent program. The work of ECO was supported by grant 510940 from the Simons Foundation. The work of IP was supported by NSF grants PHY-1804048 and PHY-1523261 and facilitated by the Max-Planck/Princeton Center for Plasma Physics. Computation of this work has been conducted at computational facilities at Princeton Institute for Computational Science and Engineering (PICSciE), and at the YZ cluster from GyroTech Co. Ltd.

\section{APPENDIX}

\section{A. DECOMPOSITION OF ALFVÉn WAVES}

Given that modifications from the CRs to the Alfvén waves are tiny in this context, it suffices to consider pure linear Alfvén waves. At fixed wavelength, there are four independent Alfvén modes, depending on left/right-handed polarization, and direction of propagation relative to fluid rest frame. We here take the perspective that the left/right handedness is defined from the receiver's point of view. That is, for a wave propagating toward an observer (relative to the fluid rest frame), the perturbed electric (and magnetic) field vector rotates in a anti-clockwise (clockwise) sense for a left (right) handed wave.

Let $\delta \boldsymbol{v}$ and $\delta \boldsymbol{B}$ be perturbed transverse velocities and magnetic fields, and take the perturbations in the form of $\exp [\mathrm{i}(k x-\omega t)]$. Note that $k$ can have both signs indicating direction of propagation, whereas $\omega$ always has positive real part. The eigenvectors for Alfvén waves satisfy

$$
\frac{\delta \boldsymbol{v}}{v_{A}}=-\operatorname{sgn}(k) \frac{\delta \boldsymbol{B}}{B_{0}} .
$$

The sign of $B_{0}$ is implicitly contained in the above, whereas $v_{A}$ is considered to be positive. In the following, we always take $B_{0}>0$ without loss of generality (for $B_{0}<0$, sign chages must be employed in many of the subsequent descriptions), and consider forward propagation to be along the direction of $\boldsymbol{B}_{0}$ (positive $\hat{x}$ ). Under this convention, the $+/-$ sign of $k$ corresponds to forward/backward propagation. The left/right-handed modes have $\delta B_{y}=\mp \mathrm{i} \delta B_{z}$ and $\delta v_{y}=\mp \mathrm{i} \delta v_{z}$ for forward-propagating waves. The opposite sign should be adopted in the above for backwardpropagating waves.

Based on the above description, the natural way to decompose circularly polarized Alfvén modes is to consider the following combination:

$$
C^{ \pm}(x)=\frac{1}{2}\left[\frac{\delta v_{y} \pm \mathrm{i} \delta v_{z}}{v_{A}}-\operatorname{sgn}(k) \frac{\delta B_{y} \pm \mathrm{i} \delta B_{z}}{B_{0}}\right] .
$$

For instance, for forward propagating $(k>0)$, right-handed $\left(\delta B_{y}=\mathrm{i} \delta B_{z}\right)$ waves, we choose the plus sign. A discrete 

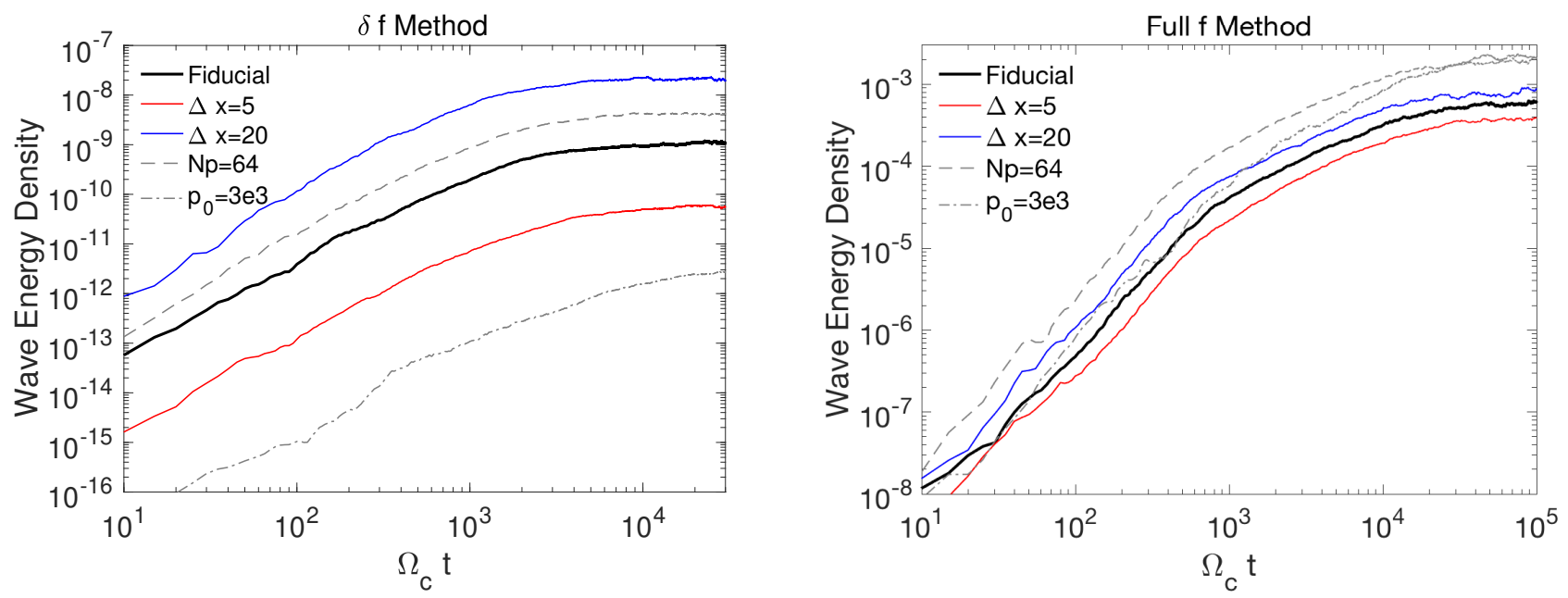

FIG. 24. - Time evolution of system noise (in terms of total wave energy density) in zero-drift simulations described in Appendix B. Left and right panels show the results from the $\delta f$ method and the full $f$ method, respectively. Bold black lines in both plots correspond to simulations with identical setup to the fiducial simulation except that $v_{D}=0$, and $n_{\mathrm{CR}} / n_{i}=10^{-3}$ for the $\delta f$ case while $n_{\mathrm{CR}} / n_{i}=10^{-4}$ in the full $f$ case. Other lines marked in the legend indicate runs where one of the parameters is varied while all others are unchanged.

Fourier transform of $C^{ \pm}(x)$,

$$
W^{ \pm}\left(k_{i}\right)=\frac{1}{N} \sum_{n=0}^{N-1} C^{ \pm}\left(x_{n}\right) e^{-\mathrm{i} k_{i} x_{n}}, \quad \text { where } k_{i}=\frac{2 \pi}{L_{x}} i,
$$

gives the wave amplitude and phase (which is what is being plotted in the Figures). The wave intensity is then given by

$$
I^{ \pm}(k)=\left|W^{ \pm}(k)\right|^{2} \frac{L_{x}}{2 \pi}
$$

To initialize a wave spectrum with $I^{ \pm}(k)=A^{2} / k$, we can simply choose $\left|W^{ \pm}(k)\right|=A \sqrt{\Delta k /|k|}$, where $\Delta k \equiv 2 \pi / L_{x}$.

\section{B. LEVEL OF NOISE IN THE $\delta F$ AND FULL $F$ METHODS}

In this Appendix, we study the noise properties in our simulations. We do so by conducting simulations with almost identical setup to our fiducial runs, except that we change the CR drift velocity to $v_{D}=0$, and that we start from an initial amplitude that is much smaller with $A=10^{-12}$. We also use a shorter simulation box $L=24000$ (which we verify has no influence on the result). In this case, we only expect wave damping to occur, but Poisson noise from particle sampling will stand out and causes fluctuations that overwhelm the initially low-amplitude waves.

In Figure 24, we show the time evolution of mean fluctuation energy density in the system, which is given by $\left(\rho v_{\perp}^{2}+B_{\perp}^{2}\right) / 2$. This is done for both the $\delta f$ method (left), and the full $f$ method (right). Note that we have chosen $n_{\mathrm{CR}} / n_{i}=10^{-3}$ in tests using the $\delta f$ method (a smaller value would take longer time to reach steady state), whereas we use $n_{\mathrm{CR}} / n_{i}=10^{-4}$ in the full $f$ case (larger value would make the noise somewhat too large). Clearly, we see that even with a higher $n_{\mathrm{CR}} / n_{i}$, the noise with the $\delta f$ method is dramatically smaller than that in the full $f$ method by more than five orders of magnitude (this would be more than six orders of magnitude if they had the same $n_{\mathrm{CR}} / n_{i}$ ).

The energy density of the waves in this test results from the Poisson noise in the particles. We can see that in both cases, reducing the number of particles by a factor of 4 leads to a factor of $\sim 4$ increase in wave energy density (or a factor $\sqrt{4}$ in wave amplitude). The wave spectrum (not shown) is consistent with white noise (with $I(k)$ being flat) for all modes with cutoff at high $k$ due to numerical damping. The spectrum at intermediate $k$ grows and saturates faster, whereas modes at the low- $k$ end grow slower, explaining the secular trend at late times.

Interestingly, the noise level also depends on resolution, but the dependence is different for the full $f$ and $\delta f$ methods. For the $\delta f$ method, we find a sensitive dependence, which scales roughly as $\Delta x^{4}$, and higher spatial resolution can greatly reduce Poisson noise. On the other hand, for the full $f$ method, the dependence is much weaker and is sublinear. In addition, we find that using a larger $p_{0}$ while keeping other parameters fixed also gives very different noise levels. Enlarging $p_{0}$ by a factor of 10 , noise is dramatically reduced by a factor of $\sim 300$ when using the $\delta f$ method. Noise is enhanced by a factor of $\sim 3-4$ when using the full- $f$ method. We do not aim at further studying and explaining these trends, but simply report them here for reference. 

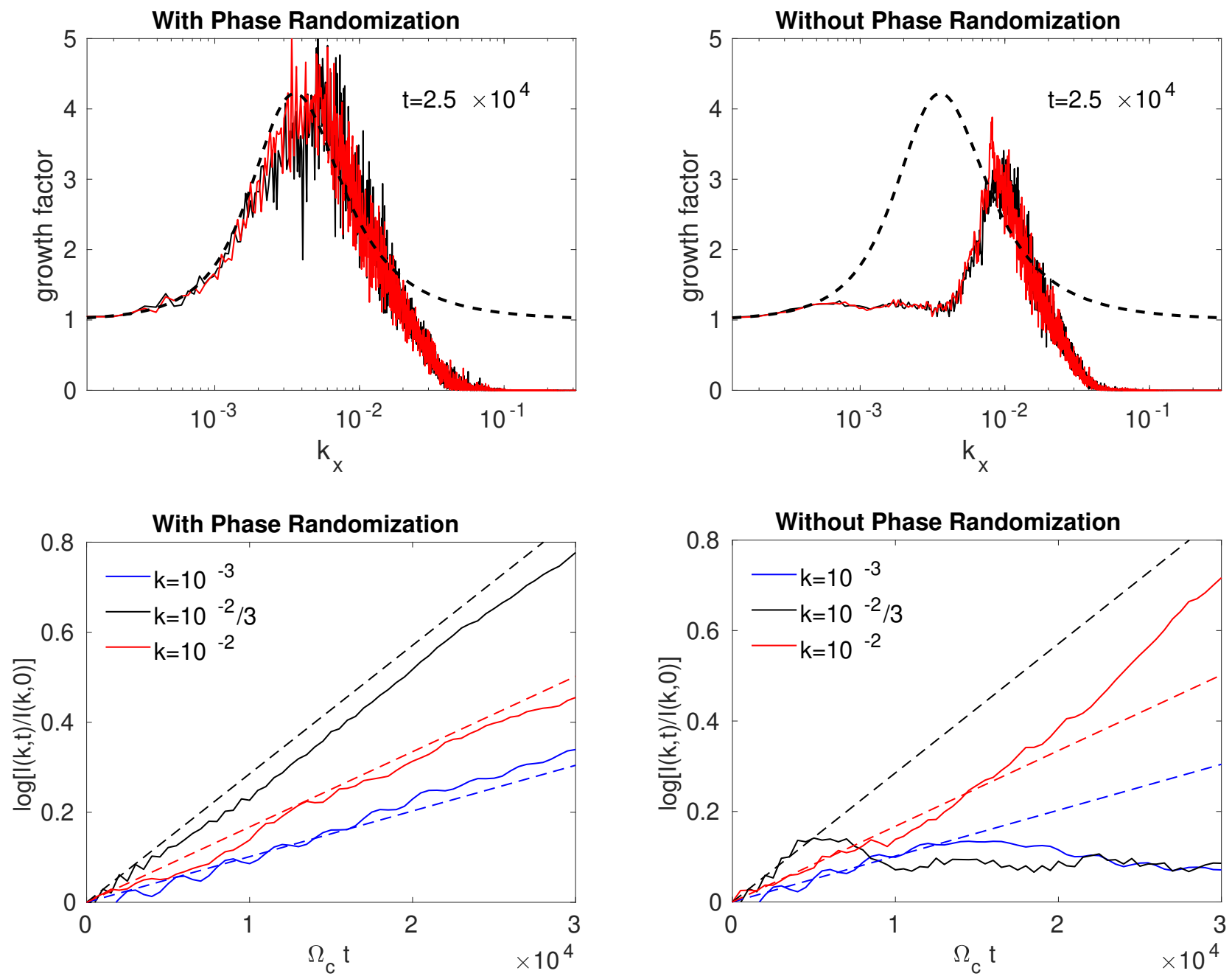

Fig. 25. - Comparison between fiducial simulations with (left) and without (right) implementing phase randomization across periodic boundaries. The top two plots show the growth factor of wave intensity $I(k)$ between $t=0$ and $t=2.5 \times 10^{4} \Omega_{c}^{-1}$, with red/black lines marking left/right handed modes. The dashed line show the growth factor expected from linear theory. The bottom two plots show the time history of growth factor for individual modes with different wave numbers $k_{x}$ (indicated in the legend). The dashed lines of the same color indicate the expected growth factor evolution.
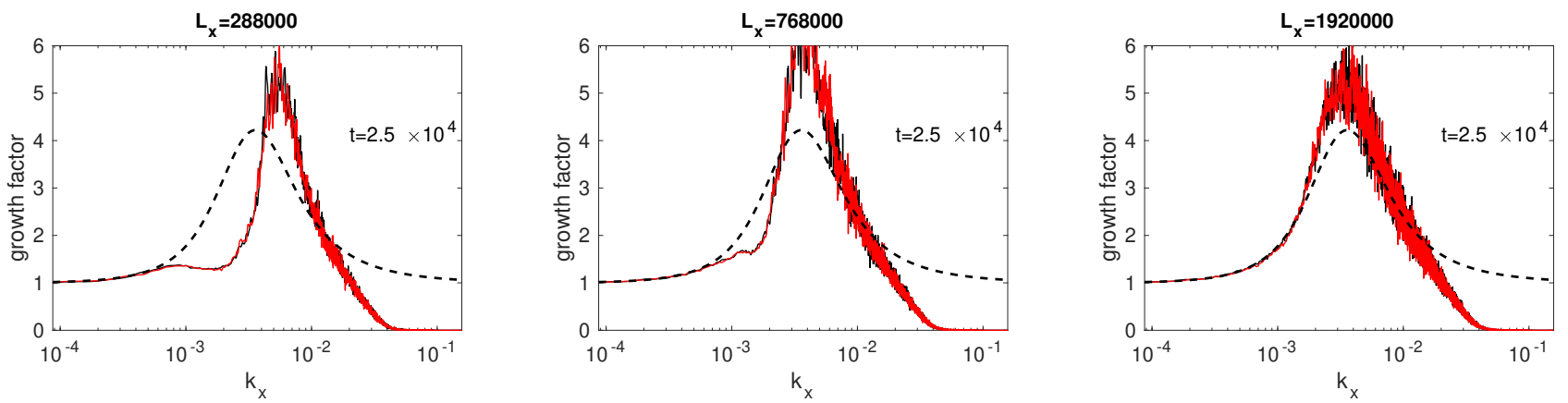

FIG. 26.- Growth factor of wave intensity $I(k)$ at time $t=2.5 \times 10^{-4}$ for three runs without phase randomization. Red/black color mark left/right handed modes. 

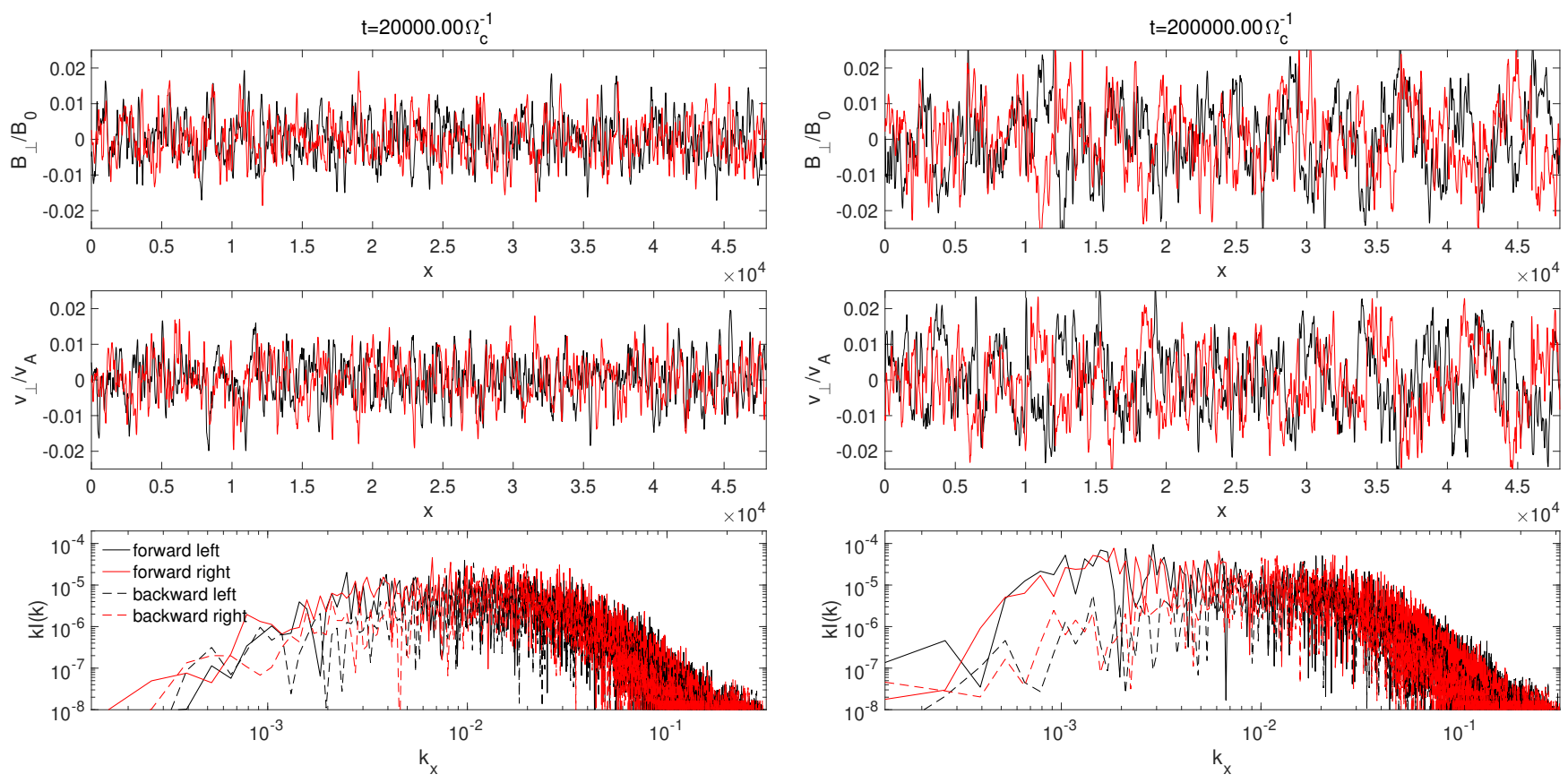

FIG. 27. - Wave pattern and their Fourier power spectrum $[k I(k)]$ at two snapshots of the fiducial simulation using the full- $f$ method: left for $t=2 \times 10^{4} \Omega_{c}^{-1}$ and right for $t=2 \times 10^{5} \Omega_{c}^{-1}$.

\section{RANDOM PHASE APPROXIMATION AND SIMULATION BOX SIZE}

In this appendix, we show that simulations without implementing phase randomization across periodic boundaries fail to properly capture the linear grow rate of the CRSI unless the simulation box size is extremely long. This failure reflects the fact that the random phase approximation must be satisfied for particles to properly follow quasi-linear diffusion, which drives the CRSI.

We first show in Figure 25 a comparison between our fiducial run with and without phase randomization. At some early time (less than one e-folding time), we plot the growth factors across the wave spectrum. Despite some slight deviation, our fiducial run with phase randomization nicely matches theoretical expectation (except for numerical damping at large $k$ ). Without phase randomization, we see that while the fastest growth rate is not different from theoretical expectation by much, the wave spectrum has significant offset, with most unstable wavelength being about a factor of three shorter.

Further looking at the time history of the wave intensity of three representative modes (with the fastest growing mode $k=(1 / 3) \times 10^{-2}$ shown in black) in the bottom two panels, we see that with phase randomization, wave growth is clearly exponential. Without randomizing the phases, the exponential growth is only maintained for up to $t \sim 5000 \Omega_{c}^{-1}$, after which the wave amplitudes wildly vary.

We then conducted a serious of simulations using fiducial parameters but in different box sizes and without phase randomization. The box sizes are $L_{x}=2.88 \times 10^{5}, 7.68 \times 10^{5}$ and $1.92 \times 10^{6}$, respectively. They are 3,8 and 20 times the length of the fiducial box that we use $\left(0.96 \times 10^{5}\right)$, while they are run for shorter amount of time (about $2.5-4 \times 10^{4} \Omega_{c}^{-1}$ ) just within the expected linear growth phase. Note that even for the longest box that we have attempted, the box crossing time for a relativistic particle is $1.92 \times 10^{6} / 300=6.4 \times 10^{3} \Omega_{c}^{-1}$, which is about a factor $\sim 4-5$ smaller than the growth time of the CRSI.

In Figure 26, we show again the spectrum of growth factors for these runs. Clearly, the offset in the fastest growing wavelength from theoretical expectation gets smaller with increasing box size, and eventually vanishes in the run with longest box size. There is still some deviation in the absolute growth rate, though only by a small factor (the numerical growth rate is accurate within about $\sim 10 \%$ ). We thus consider this longest box size run to be marginally appropriate to study the CRSI.

\section{COMPARISON WITH THE FULL-F METHOD}

To demonstrate the advantage of the $\delta f$ method, we show in this Appendix results obtained from the full $f$ approach. The simulation setup is identical to our run Fid except that the $\delta f$ weighting scheme is turned off and that the box size is halved. Phase randomization is also included. To further reduce Poisson noise, we use $N_{p}=2048$ particles per bin per cell (in total 16384 per cell). According to the study in Appendix B, the level of noise is reduced by a factor of 8 in wave intensity, with wave energy density $\sim 10^{-4}$, or $\delta B^{2} / B_{0}^{2} \lesssim 5 \times 10^{-5}$, in the form of Alfvén waves 


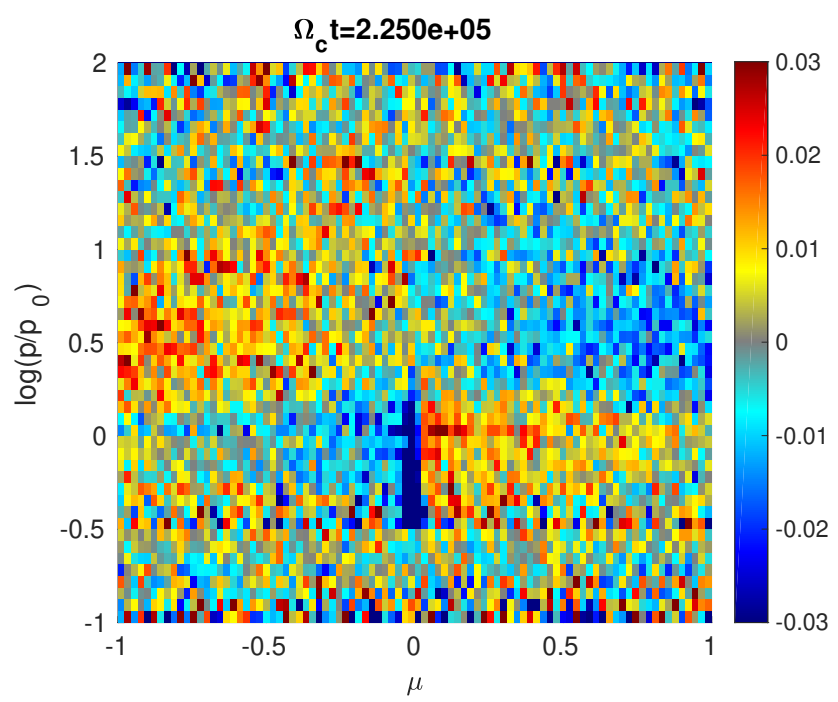

FIG. 28. - The distribution function $\left(f-f_{0}\right) / f_{0}$ at the last snapshot $t=2.25 \times 10^{5} \Omega_{c}^{-1}$ from the fiducial simulation using the full- $f$ method.

propagating in both directions. This level of noise would yield a rate of QLD, according to (15), that is comparable to the maximum wave growth rate of $\sim 3 \times 10^{-5} \Omega_{c}$. The simulation is run to time $t=2.25 \times 10^{5} \Omega_{c}^{-1}$.

In Figure 27, we show the two snapshots the wave pattern and spectrum. We see that noise level indeed quickly reaches the level of $\delta B / B \lesssim 10^{-2}$, causing appreciable QLD of particles. Note that since Alfvén waves in both directions are present due to noise, CR energy would no longer be conserved in the (forward-propagating) wave frame, compromising the physics of the CRSI. As a result, we can only barely identify the signature of the CRSI in the wave spectrum at early time of $t=2 \times 10^{4}$. At later time, forward-propagating waves are more easily seen to be amplified. However, these waves are at longer wavelengths (with $k \sim 10^{-3}$ instead of $\Omega_{c} / p_{0}=3.3 \times 10^{-3}$ ) where intrinsic numerical noise is smaller, allowing the CRSI to better stand out. The growth rates at these longer wavelength are somewhat too noisy to measure with sufficient precision to compare with theoretical values, and through our analysis, particles responsible for wave growth must have already undergone some QLD from pure noise.

We have further inspected the evolution of the distribution function, and show in Figure 28 this distribution function for the last snapshot of the run. Note that because the binning is based on the full- $f$ prescription (i.e., direct particle counting in each bin), it is much noisier than we had before under the $\delta f$ prescription. On the other hand, we can find signatures of isotropization. In fact, particles at $p \sim 3 p_{0}$ are already nearly isotropized, and particles with $p \gtrsim 10 p_{0}$ are still in early stages of isotropization. For particles with $p \lesssim p_{0}$, accumulation near $\mu \sim 0$ is also clearly visible, as they are stuck and can not cross the $90^{\circ}$ pitch angle. While these features are qualitatively consistent with expectations, QLD proceeds much faster than that obtained using the $\delta f$ method owing to substantial enhancement of scattering through noise. In particular, recall from discussions in Section 5.2, isotropization is never achieved when using the $\delta f$ method.

Overall, we conclude that while the full- $f$ method can capture some basic properties of the CRSI, numerical noise adversely affects its evolution at all stages, leading to severe discrepancies at a quantitative (and sometimes even qualitative) level, which precludes making any accurate/reliable measurement. The situation can only get worse under more realistic conditions (low $n_{\mathrm{CR}} / n_{i}$ and small $v_{D}$ ).

\section{REFERENCES}

Achterberg, A. 1981, A\&A, 98, 161

Amato, E., \& Blasi, P. 2009, MNRAS, 392, 1591

Amato, E., \& Blasi, P. 2018, Advances in Space Research, 62, 2731

Baade, W. \& Zwicky, F. 1934, Proceedings of the National Academy of Science, 20, 259

Bai, X.-N., Caprioli, D., Sironi, L., \& Spitkovsky, A. 2015, ApJ, 809, 55

Bell, A. R. 1978, MNRAS, 182, 147

-. 2004, MNRAS, 353, 550

Birdsall, C. K. \& Langdon, A. B. 2005, Plasma Physics Via Computer Simulation (Taylor \& Francis Group, 2005)

Blandford, R. D. \& Ostriker, J. P. 1978, ApJ, 221, L29

Blasi, P., Amato, E., \& Serpico, P. D. 2012, Physical Review Letters, 109, 061101

Booth, C. M., Agertz, O., Kravtsov, A. V., \& Gnedin, N. Y. 2013, ApJ, 777, L16
Boris, J. P. 1970, in Proceedings of the Fourth Conference on Numerical Simulation Plasmas (Navel Research Laboratory, Washington, D.C.), pp. 3-67

Breitschwerdt, D., McKenzie, J. F., \& Voelk, H. J. 1991, A\&A, 245, 79

Breitschwerdt, D., McKenzie, J. F., \& Voelk, H. J. 1993, A\&A, 269,54

Denton, R. E. \& Kotschenreuther, M. 1995, Journal of Computational Physics, 119, 283

Dimits, A. M. \& Lee, W. W. 1993, Journal of Computational Physics, 107, 309

Draine, B. T. 2011, Physics of the Interstellar and Intergalactic Medium by Bruce T. Draine. Princeton University Press, 2011. ISBN: 978-0-691-12214-4,

Drury, L. O. 1983, Reports on Progress in Physics, 46, 973

Dupree, T. H. 1966, Physics of Fluids, 9, 1773 
Enßlin, T. A., Pfrommer, C., Springel, V., \& Jubelgas, M. 2007, A\&A, 473, 41

Enßlin, T., Pfrommer, C., Miniati, F., \& Subramanian, K. 2011, A\&A, 527, A99

Everett, J. E., Zweibel, E. G., Benjamin, R. A., McCammon, D., Rocks, L., \& Gallagher, III, J. S. 2008, ApJ, 674, 258

Farber, R., Ruszkowski, M., Yang, H.-Y. K., \& Zweibel, E. G. 2018, ApJ, 856, 112

Farmer, A. J. \& Goldreich, P. 2004, ApJ, 604, 671

Felice, G. M. \& Kulsrud, R. M. 2001, ApJ, 553, 198

Ferrière, K. M. 2001, Reviews of Modern Physics, 73, 1031

Fujita, Y., \& Ohira, Y. 2011, ApJ, 738, 182

Gardiner, T. A. \& Stone, J. M. 2005, Journal of Computational Physics, 205, 509

—. 2008, Journal of Computational Physics, 227, 4123

Ginzburg, V. L. \& Syrovatskii, S. I. 1964, The Origin of Cosmic Rays

Girichidis, P., Naab, T., Walch, S., Hanasz, M., Mac Low, M.-M., Ostriker, J. P., Gatto, A., Peters, T., Wünsch, R., Glover, S. C. O., Klessen, R. S., Clark, P. C., \& Baczynski, C. 2016 ApJ, 816, L19

Girichidis, P., Naab, T., Hanasz, M., \& Walch, S. 2018, MNRAS, 479, 3042

Goldreich, P. \& Sridhar, S. 1995, ApJ, 438, 763

Guo, F. \& Oh, S. P. 2008, MNRAS, 384, 251

Grenier, I. A., Black, J. H., \& Strong, A. W. 2015, ARA\&A, 53, 199

Hanasz, M., Lesch, H., Naab, T., Gawryszczak, A., Kowalik, K., \& Wóltański, D. 2013, ApJ, 777, L38

Holcomb, C. \& Spitkovsky, A. 2018, arXiv e-prints

Holguin, F., Ruszkowski, M., Lazarian, A., Farber, R., \& Yang, H.-Y. K. 2018, arXiv:1807.05494

Hu, G. \& Krommes, J. A. 1994, Physics of Plasmas, 1, 863

Ipavich, F. M. 1975, ApJ, 196, 107

Jacob, S., \& Pfrommer, C. 2017, MNRAS, 467, 1449

Jacob, S., Pakmor, R., Simpson, C. M., Springel, V., \& Pfrommer, C. 2018, MNRAS, 475, 570

Jiang, Y.-F. \& Oh, S. P. 2018, ApJ, 854, 5

Jokipii, J. R. 1966, ApJ, 146, 480

Jubelgas, M., Springel, V., Enßlin, T., \& Pfrommer, C. 2008, A\&A, 481, 33

Krymskii, G. F. 1977, Akademiia Nauk SSSR Doklady, 234, 1306

Kulsrud, R. \& Pearce, W. P. 1969, ApJ, 156, 445

Kulsrud, R. M. 2005, Plasma physics for astrophysics (Princeton University Press)

Kunz, M. W., Stone, J. M., \& Bai, X.-N. 2014, Journal of Computational Physics, 259, 154

Lazarian, A. 2016, ApJ, 833, 131

Lazarian, A., \& Beresnyak, A. 2006, MNRAS, 373, 1195
Lebiga, O., Santos-Lima, R., \& Yan, H. 2018, MNRAS, 476, 2779

Lee, M. A. \& Völk, H. J. 1973, Ap\&SS, 24, 31

Mao, S. A., \& Ostriker, E. C. 2018, ApJ, 854, 89

Naab, T., \& Ostriker, J. P. 2017, ARA\&A, 55, 59

Pakmor, R., Pfrommer, C., Simpson, C. M., \& Springel, V. 2016, ApJ, 824, L30

Parker, E. N. 1966, ApJ, 145, 811

-. 1992, ApJ, 401, 137

Parker, S. E. \& Lee, W. W. 1993, Physics of Fluids B, 5, 77

Pfrommer, C., Pakmor, R., Schaal, K., Simpson, C. M., \& Springel, V. 2017, MNRAS, 465, 4500

Recchia, S., Blasi, P., \& Morlino, G. 2016, MNRAS, 462, 4227

Roe, P. L. 1981, Journal of Computational Physics, 43, 357

Ruszkowski, M., Yang, H.-Y. K., \& Zweibel, E. 2017, ApJ, 834, 208

Salem, M. \& Bryan, G. L. 2014, MNRAS, 437, 3312

Salem, M., Bryan, G. L., \& Hummels, C. 2014, ApJ, 797, L18

Schlickeiser, R. 1989, ApJ, 336, 264

Schlickeiser, R. \& Miller, J. A. 1998, ApJ, 492, 352

Sharma, P., Colella, P., \& Martin, D. F. 2010, SIAM J Sci. Comp. 32,3564

Simpson, C. M., Pakmor, R., Marinacci, F., et al. 2016, ApJ, 827, L29

Skilling, J. 1975a, MNRAS, 172, 557

-. 1975b, MNRAS, 173, 245

-. 1975c, MNRAS, 173, 255

Soler, R., Terradas, J., Oliver, R., \& Ballester, J. L. 2016, A\&A, 592, A28

Stone, J. M., Gardiner, T. A., Teuben, P., Hawley, J. F., \&

Simon, J. B. 2008, ApJS, 178, 137

Summers, D. \& Thorne, R. M. 1991, Physics of Fluids B, 3, 1835

Thomas, T. \& Pfrommer, C. 2018, arXiv e-prints

Uhlig, M., Pfrommer, C., Sharma, M., Nath, B. B., Enßlin, T. A., \& Springel, V. 2012, MNRAS, 423, 2374

Völk, H. J. 1973, Ap\&SS, 25, 471

Wentzel, D. G. 1974, ARA\&A, 12, 71

Wiener, J., Oh, S. P., \& Guo, F. 2013, MNRAS, 434, 2209

Wiener, J., Pfrommer, C., \& Oh, S. P. 2017, MNRAS, 467, 906

Wiener, J., Zweibel, E. G., \& Oh, S. P. 2018, MNRAS, 473, 3095

Wiener, J., \& Zweibel, E. G. 2018, arXiv:1812.02179

Yan, H. \& Lazarian, A. 2002, Physical Review Letters, 89, 281102 -. 2008, ApJ, 673, 942

Zirakashvili, V. N., Breitschwerdt, D., Ptuskin, V. S., \& Voelk,

H. J. 1996, A\&A, 311, 113

Zweibel, E. G. 2013, Physics of Plasmas, 20, 055501

Zweibel, E. G. 2017, Physics of Plasmas, 24, 055402 